\title{
Real-time renormalization group in frequency space: A two-loop analysis of the nonequilibrium anisotropic Kondo model at finite magnetic field
}

\author{
Herbert Schoeller ${ }^{1,2}$ and Frank Reininghaus ${ }^{1,2}$ \\ ${ }^{1}$ Institut für Theoretische Physik, Lehrstuhl A, RWTH Aachen, 52056 Aachen, Germany \\ ${ }^{2}$ JARA-Fundamentals of Future Information Technology
}

(Dated: October 27, 2018)

\begin{abstract}
We apply a recently developed nonequilibrium real-time renormalization group (RG) method in frequency space to describe nonlinear quantum transport through a small fermionic quantum system coupled weakly to several reservoirs via spin and/or orbital fluctuations. Within a weakcoupling two-loop analysis, we derive analytic formulas for the nonlinear conductance and the kernel determining the time evolution of the reduced density matrix. A consistent formalism is presented how the RG flow is cut off by relaxation and dephasing rates. We apply the general formalism to the nonequilibrium anisotropic Kondo model at finite magnetic field. We consider the weakcoupling regime, where the maximum of voltage and bare magnetic field is larger than the Kondo temperature. In this regime, we calculate the nonlinear conductance, the magnetic susceptibility, the renormalized spin relaxation and dephasing rates, and the renormalized $g$ factor. All quantities are considered up to the first logarithmic correction beyond leading order at resonance. Up to a redefinition of the Kondo temperature, we confirm previous results for the conductance and the magnetic susceptibility in the isotropic case. In addition, we present a consistent calculation of the resonant line shapes, including the determination whether the spin relaxation or dephasing rate cuts off the logarithmic divergence. Furthermore, we calculate quantities characterizing the exponential decay of the time evolution of the magnetization. In contrast to the conductance, we find that the derivative of the spin relaxation (dephasing) rate with respect to the magnetic field is logarithmically enhanced (suppressed) for voltages smaller (larger) than the renormalized magnetic field, and that the logarithmic divergence is cut off by the opposite rate. The renormalized $g$ factor is predicted to show a symmetric logarithmic suppression at resonance, which is cut off by the spin relaxation rate. We propose a three-terminal setup to measure the suppression at resonance. For all quantities, we analyze also the anisotropic case and find additional nonequilibrium effects at resonance.
\end{abstract}

PACS numbers: $05.10 . \mathrm{Cc}, 72.10 . \mathrm{Bg}, 73.63 . \mathrm{Nm}$

\section{INTRODUCTION}

One of the basic unsolved problems of dissipative quantum mechanics and quantum transport through mesoscopic systems is the nonequilibrium Kondo model. In its simplest version, it consists of a spin- $\frac{1}{2}$ system coupled via exchange processes to the spins of two fermionic reservoirs, which are kept at two different chemical potentials $\mu_{L / R}= \pm e \frac{V}{2}$, see Fig. 1. Besides the importance of the Kondo model for many aspects of strongly correlated Fermion systems (see Ref. 1 for an overview), it was suggested to realize this model in transport experiments through quantum dots $\underset{2}{2}$ This has been achieved $\underline{3}$ with the particular advantage of full control over all parameters such as temperature, voltage, magnetic field, and exchange couplings. The central idea is to lower temperature and bias voltage such that only one singleparticle level of a quantum dot will contribute to transport. Adjusting the gate voltage such that charge fluctuations of this level are suppressed (Coulomb blockade regime), the dot can either be occupied by a spin up or a spin down electron, and the spin can fluctuate via second-order cotunneling processes, leading precisely to the model depicted in Fig. 1. In this realization, one obtains an antiferromagnetic exchange coupling of the order $J \sim \rho t^{2} / E_{c}$ (in dimensionless units), where $\rho$ is the density of states in the reservoirs, $t$ denotes the tunneling

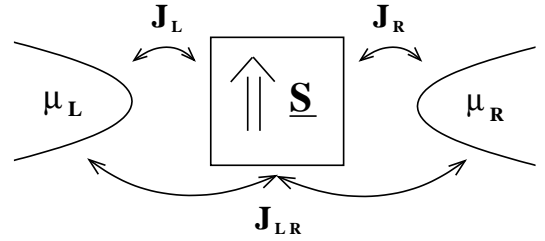

FIG. 1: A spin- $\frac{1}{2}$ quantum system coupled via exchange to two reservoirs. $J_{L}=J_{L L}$ and $J_{R}=J_{R R}$ involve exchange between the spins of the left/right reservoir with the local spin, and $J_{L R}=J_{R L}$ transfers a particle from one reservoir to the other during the exchange process.

amplitude (hopping parameter) between the leads and the dot, and $E_{c}$ is the energy of the virtual intermediate state (charging energy). In equilibrium, the Kondo model has been analyzed by various many-body methods (for an overview, see Ref. 1) and can be solved exactly by Bethe ansatz ${ }^{\underline{4}}$ or conformal field theory. ${ }^{\underline{5}}$ Powerful numerical techniques such as the numerical renormalization group have been developed ${ }^{6.7}$ from which all thermodynamic and spectral properties can be calculated. The basic physics can already be understood from poor man scaling arguments $\stackrel{8}{=}$ If all exchange couplings are the same $J_{L}=J_{R}=J_{L R}=J_{0}$, one obtains the renormaliza- 
tion group $(\mathrm{RG})$ equation

$$
\frac{d J}{d l}=2 J^{2} \quad, \quad l=\ln \frac{D}{\Lambda},
$$

with the solution

$$
J=\frac{1}{2 \ln \frac{\Lambda}{T_{K}}} \quad, \quad T_{K}=D e^{-\frac{1}{2 J_{0}}} .
$$

Here, $T_{K}$ is the Kondo temperature and $J$ denotes an effective exchange coupling corresponding to an effective band width $\Lambda$ of the reservoirs $\left(D \sim E_{c}\right.$ is the original band width). For the antiferromagnetic case $J>0$, one obtains an enhancement of the exchange coupling by reducing the effective band width until $\Lambda$ reaches $T_{K}$, where $J$ diverges. This indicates a logarithmic enhancement of the linear conductance for temperatures above the Kondo temperature (weak-coupling regime), and a complete screening of the dot spin by the reservoir spins below $T_{K}$ (strong-coupling regime). In the latter case, it can be shown that the conductance becomes unitary, i.e., $G=2 e^{2} / h$, at zero temperature $T=0$ and zero bias voltage $V=0$.

In nonequilibrium, the Kondo model is not yet solved completely. Some numerical techniques have already been developed to describe either the time evolution of an out-of-equilibrium initial state by using time-dependent numerical renormalization group (TD-NRG) (Ref. 9) or to describe the stationary current in the presence of a bias by using NRG in a scattering wave basis $\frac{10}{10}$ or using an iterative real-time path integral approach. ${ }^{11}$ Scattering wave Bethe ansatz methods based on the LippmannSchwinger equation are currently under way to solve the single-impurity Anderson model in nonequilibrium. ${ }^{12}$ Concerning analytical RG methods, it has been emphasized that it is important to understand how the RG equations are cut off by the bias voltage and by relaxation and dephasing rates $\frac{13,14,15}{15}$ The problem can be stated as follows. Performing perturbation theory in the bare exchange coupling $J_{0}$ (disregarding for the moment the differences between $J_{L}, J_{R}$, and $J_{L R}$ ), logarithmic terms occur, which, at zero temperature and depending on the physical observable under consideration, have the form [we indicate only the order in the exchange coupling leaving out numbers of $O(1)$ and other physical energy scales from $V$ and $h_{0}$ in the prefactor]

$$
\left\{\begin{array}{c}
I, \tilde{\Gamma}_{1}, \tilde{\Gamma}_{2} \\
\tilde{g}-2 \\
M
\end{array}\right\} \rightarrow\left\{\begin{array}{c}
J_{0}^{2} \\
J_{0} \\
1
\end{array}\right\} J_{0}^{k} \ln ^{l} \frac{D}{\left|n V-m h_{0}\right|},
$$

with $k \geq l \geq 0, n=0, \pm 1, \pm 2, \ldots$ and $m=0, \pm 1$. Here, $I$ is the current, $\tilde{\Gamma}_{1 / 2}$ are the spin relaxation/dephasing rates, $\tilde{g}$ is the renormalized $g$ factor, and $M$ denotes the magnetization. $D$ is the band width of the reservoirs and $h_{0}$ is the bare magnetic field. $n$ denotes the number of particles transferred between the reservoirs and $m=0, \pm 1$ characterizes whether spin flip processes occur or not. The points $n V=m h_{0}$ correspond to resonance positions, where certain higher-order processes are allowed by energy conservation, e.g., the value $n=m=1$ corresponds to the onset of inelastic cotunneling processes $\underline{16}$ Higher-order terms with $n \geq 2$ have so far not been discussed in the literature for the Kondo model, but are generically expected ${ }^{17}$ (for other models in the charge fluctuation regime, corresponding terms have been calculated in Ref. 18). For given perturbation order $k$, the allowed values for $l$ depend on the value of $n$. For $n=0,1$ it is known that $l=0,1, \ldots, k$. Even in the weak-coupling regime

$$
\Lambda_{c}=\max \left\{V, h_{0}\right\} \gg T_{K},
$$

the logarithmic terms can lead to a breakdown of perturbation theory if $J_{0} \ln \frac{\Lambda_{c}}{T_{K}}$ is small enough such that $J_{0} \ln \frac{D}{\Lambda_{c}}=\frac{1}{2}-J_{0} \ln \frac{\Lambda_{c}}{T_{K}} \sim O(1)$. Furthermore, at resonance $n V=m h_{0}$, the logarithmic terms even diverge. Therefore, it is necessary to resum the logarithmic terms in an appropriate way using RG and, at the same time, introduce the physics of relaxation and dephasing rates to cut off the divergencies at resonance. This idea has been proposed in Ref. 19. In a first step, within a standard poor man scaling approach, one resums all leading order logarithmic terms of the form

$$
J_{0}^{r}\left(J_{0} \ln \frac{D}{\Lambda_{c}}\right)^{k} \quad, \quad k=0,1,2, \ldots,
$$

where $r=0,1,2$ depends on the physical observable under consideration [see Eq. (3)]. This leads to an effective exchange coupling $J_{c}$, given by Eq. (2) evaluated at $\Lambda=\Lambda_{c}$ :

$$
J_{c}=\frac{1}{2 \ln \frac{\Lambda_{c}}{T_{K}}}=\frac{1}{2 \ln \frac{\max \left\{V, h_{0}\right\}}{T_{K}}} .
$$

In a second step, one tries to expand the physical observable systematically in powers of the effective coupling constant $J_{c}$, leading to a series with terms similiar to Eq. (3), but with the replacements $D \rightarrow \Lambda_{c}$ and $J_{0} \rightarrow J_{c}$. If, in addition, one cuts off the resonances by an appropriate relaxation or dephasing rate $\tilde{\Gamma}$, a new series of the form

$$
J_{c}^{k+r} \ln ^{l} \frac{\Lambda_{c}}{|n V-m \tilde{h}+i \tilde{\Gamma}|}
$$

is expected, where $\tilde{h}$ is the renormalized magnetic field. As pointed out in Ref. 19 this perturbation series in $J_{c}$ is well-defined for $J_{c} \ll 1$, because the maximum value of the logarithm at resonance is given by $\ln \frac{\Lambda_{c}}{\Gamma} \sim \ln J_{c}$, where we have used the rough estimate

$$
\tilde{\Gamma} \sim J_{c}^{2} \Lambda_{c}
$$

based on a simple dimensional analysis. Therefore, at resonance, we expect terms of the form

$$
J_{c}^{k+r} \ln ^{l} J_{c},
$$


which are not dangerous since $J_{c} \ln J_{c} \ll 1$ if $J_{c} \ll 1$ (note that $k \geq l$ ). The leading order result is the term $\sim$ $J_{c}^{r}$ (denoted as one-loop in this paper), whereas the first logarithmic correction $\sim J_{c}^{r+1} \ln J_{c}$ is the first subleading term (denoted as two-loop in this paper).

The purpose of the present paper is to present a welldefined two-loop RG approach to work out the abovedescribed procedure. Thereby we will apply a recently proposed real-time renormalization group method in frequency space (RTRG-FS) $\stackrel{17}{=}$ This method has the advantage that formally exact $R G$ equations can be set up in nonequilibrium which include the relaxation and dephasing rates in all resolvents appearing on the right hand side (r.h.s.) of the RG equations. Furthermore, in leading order, precisely poor man scaling equation (1) is obtained. This provides the possibility to proceed in two steps: First one expands the exact RG equations systematically around the poor man scaling solution for $D>\Lambda>\Lambda_{c}$, and, in the second step, one solves the RG equations perturbatively in $J_{c}$ for $\Lambda_{c}>\Lambda>0$. As we will show in this paper, in both steps two-loop terms are important to obtain the first logarithmic corrections beyond leading order. In the first step, two-loop terms arising from higher-order terms on the r.h.s. of the RG equation generate terms $\sim J_{c}^{r+1} \ln \frac{J_{c}}{J_{0}}$ which depend only weakly on the voltage and are incorporated into a redefinition of the coupling constant $J_{c}$ (or, equivalently, the Kondo temperature). These terms lead to an overall increase (or decrease) in the physical observable under consideration but show no interesting dependence on voltage or magnetic field. In contrast, in the second step, logarithmic contributions of form (7) are generated which give rise to a logarithmic enhancement (suppression) at resonance. This means that the various two-loop terms (leading all to terms of the same order of magnitude) can be systematically divided into important and unimportant terms concerning their dependence on voltage and magnetic field. In accordance with results of Refs. 15 and 20, we emphasize that it is very important to include the frequency dependence of the vertices generated from the first step $D>\Lambda>\Lambda_{c}$ since this influences the prefactor of the logarithmic contributions calculated in the second step $\Lambda_{c}>\Lambda>0$.

In one-loop order (but including certain two-loop terms from the frequency dependence of the vertices), the conductance and the magnetization have been calculated previously for the nonequilibrium Kondo model. Pioneering works are Refs. 15 and 20, where the slave particle approach was used in combination with the Keldysh formalism and quantum Boltzmann equations. The RG was formulated only on one part of the Keldysh contour and a real-frequency cutoff was used. In these works, it was investigated how the voltage and the magnetic field cut off the RG flow and it was emphasized that the frequency dependence of the vertices is important to obtain the first logarithmic contributions beyond leading order. In fact, the result of the present paper concerning the conductance and the magnetization is precisely the same as that of Refs. 15 and 20, up to the redefinition of the Kondo temperature. This means that we will prove in this paper that all two-loop contributions neglected in Refs. 15 and 20 do not influence the prefactor of the first logarithmic correction beyond leading order. Furthermore, in Refs. 15 and 20, diagrams connecting the upper with the lower part of the Keldysh contour have been neglected. Within these works, it was therefore not possible to describe the cutoff of the logarithmic terms by relaxation and dephasing rates on a full microscopic level. In this paper, we will show how this can be achieved within RTRG-FS, which provides a consistent formalism to calculate the line shape at resonance and to see whether the spin relaxation rate $\tilde{\Gamma}_{1}$ or the spin dephasing rate $\tilde{\Gamma}_{2}$ cuts off the logarithmic divergencies (in Ref. 29 , the latter question was adressed only in bare perturbation theory and zero magnetic field). In addition, in this paper we will also discuss the anisotropic case and calculate the spin relaxation/dephasing rates together with the renormalized $g$ factor up to the first logarithmic contribution (corresponding to a three-loop calculation in conventional RG methods). Besides the known reduction in the magnetic field in first order in $J, 33$ we find that the renormalized magnetic field in second order in $J$ is proportional to logarithmic terms similiar to Eq. (7) with a significant dependence on voltage and magnetic field. We propose an experimental setup with a weakly coupled third lead to measure the voltage dependence of the renormalized $g$ factor. Moreover, we find that the logarithmic terms of $\tilde{\Gamma}_{2}$ and $\tilde{g}$ are controlled by $\tilde{\Gamma}_{1}$, whereas those of $\tilde{\Gamma}_{1}$ are controlled by $\tilde{\Gamma}_{2}$. In the anisotropic case this leads to the effect that the logarithmic resonances of $\frac{d \tilde{\Gamma}_{2}}{h_{0}}$ become sharper with decreasing $J^{\perp}$ since $\tilde{\Gamma}_{1}$ does not contain any terms proportional to $J^{z}$ in second order. Furthermore, we will show that the susceptibility depends only weakly on the tranverse coupling $J^{\perp}$ and the logarithmic resonances even survive in the limit $J^{\perp} \rightarrow 0$. The anisotropic Kondo model has recently been proposed to be realizable in low-temperature transport through single molecular magnets 21 and experiments are starting to investigate such systems $\stackrel{22}{=}$ Since the transverse coupling is induced by small magnetic quantum tunneling terms, giving rise to rather small Kondo temperatures, the susceptibility might be an interesting physical quantity to measure signatures of the Kondo effect even for very small values of $J^{\perp}$.

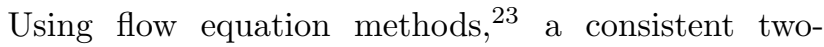
loop approach including the cutoff by spin relaxation/dephasing rates has been presented in Ref. 24 for the isotropic Kondo model in the absence of a magnetic field. Within this method, the cutoff from the rate $\tilde{\Gamma}$ occurs due to a competition of certain one-loop and twoloop terms on the r.h.s. of the RG equation for the vertex. This is a completely different picture compared to RTRG-FS, where the cutoff parameter $\tilde{\Gamma}$ together with the voltage occurs already in the one-loop terms as an additional term in the denominator of the resolvents. Thus, the RTRG-FS method is closer to conventional poor man 
scaling and the physics of relaxation and dephasing rates occurs naturally as a resummation of a geometric series similiar to self-energy insertions in Green's function techniques. In this sense the RTRG-FS method proves that conventional scaling equations (properly generalized to the Keldysh contour) can account for the cutoff of the RG flow by rates and the voltage. Furthermore, the RTRG-FS method provides a generic proof in all orders of perturbation theory in the renormalized vertices that the various cutoff scales $\tilde{\Gamma}_{k}$ are the physical relaxation and dephasing rates governing the time evolution of the reduced density matrix of the quantum system.

The RTRG-FS method used in this paper has been proposed in Ref. 17 and is a natural generalization of an earlier developed real-time RG method: ${ }^{25,26,27}$ As described in detail in Ref. 17, many technical improvements have been incorporated, the main ones being a formulation of the RG in pure frequency space, integrating out the symmetric part of the Fermi distribution function before starting the RG, and formulating the nonequilibrium $\mathrm{RG}$ on the imaginary frequency axis. As a consequence, the rates determining the cutoff of the $\mathrm{RG}$ flow obtain the right scale, and it is possible to show generically that relaxation and dephasing rates cut off the $\mathrm{RG}$ flow in all orders of perturbation theory and within all truncation schemes. Furthermore, the dependence on the Keldysh indices can be completely avoided, and one can calculate the time evolution and the nonequilibrium stationary state in pure Matsubara space without the need of any analytic continuation. The latter idea has first been proposed in Ref. 28 in the context of nonequilibrium functional renormalization group within the Keldysh formalism. A particular advantage of the RTRG-FS method is that relaxation and dephasing rates occur naturally as the negative imaginary part of the eigenvalue of the kernel determining the kinetic equation of the reduced density matrix, and do not arise from more involved combinations of self-energies and vertex corrections as in slave particle formalism. ${ }^{29}$ Furthermore, it is straightforward to calculate the time evolution from RTRG-FS since the $\mathrm{RG}$ gives directly the result for the kernel in Laplace space.

For generic problems with spin and/or orbital fluctuations, it was described in Ref. 17 how to solve the RG equations analytically in the weak coupling regime up to one-loop order. In this paper we will provide the technically much more involved two-loop case, which is necessary to calculate consistently the important logarithmic terms at resonance discussed above, see Eq. (7). The result will be applied to the calculation of the conductance and the magnetic susceptibility of the anisotropic Kondo model in the presence of a magnetic field. Furthermore, we will also analyze quantities characterizing the time evolution of the Kondo model. Thereby, we will concentrate on the calculation of the dominant exponential decay of the magnetization in two-loop order, which is determined by the spin relaxation and dephasing rates $\tilde{\Gamma}_{1,2}$ and the renormalized magnetic field $\tilde{h}$. Finally, in addition to Ref. 17, we will generically show that precisely these physical quantities control all resonant line shapes (in Ref. 17, the question whether the cutoff scales of the logarithmic terms are exactly identical to the physical relaxation/dephasing rates was still open).

The paper is organized as follows. In Sec. II A, we will set up the generic model and the perturbative series. Section $\Pi \mathrm{II}$ is devoted to the general RG formalism and the derivation of the two-loop RG equations for an arbitrary quantum dot coupled via spin and/or orbital fluctuations to reservoirs. The systematic way to analytically solve these RG equations up to two-loop order is presented in Sec. IIC. The general formalism is applied to the nonequilibrium Kondo model in Sec. III. In Sec.III A, we will set up the algebra in Liouville space needed to evaluate all expressions explicitly, and Sec. IIIB describes the evaluation of the general two-loop equations for the Kondo model. The final results for the conductance, the magnetic susceptibility, the spin relaxation and dephasing rate, and the renormalized $g$ factor are presented for the isotropic case in Secs. IVA, IVB and IVC, whereas the anisotropic case is discussed in section IVD. We close with a summary in Sec. V. A list of all symbols used in this papers is presented in Sec. VI

We emphasize that a reader, who is not interested in the formal derivation but only in the final physical results of the paper, can skip the following formal Secs. II] and III, and can directly move over to Sec. IV] where the notations needed to understand the results are again repeated.

\section{GENERIC CASE}

In this section, we describe a generic quantum dot coupled via spin and/or orbital fluctuations to several reservoirs. In Secs. II A and II B, we introduce the basic notations, the perturbative series and summarize shortly the setup of the RG equations as explained in more detail in Ref. 17. In Sec. II C] we present a systematic way how to solve the RG equations analytically up to two-loop order in the weak-coupling regime (the one-loop case has been treated in Ref. 17). Throughout this paper, we use units $e=\hbar=k_{B}=1$.

\section{A. Model and perturbative series}

Model. We consider a quantum dot with fixed charge in the Coulomb blockade regime where only spin and/or orbital fluctuations are possible via the coupling to external reservoirs. As shown in detail in Ref. 27, a standard Schrieffer-Wolff transformation leads to a Hamiltonian of the form

$$
H=H_{\text {res }}+H_{S}+V=H_{0}+V,
$$

where $H_{r e s}$ is the reservoir part, $H_{S}$ characterizes the isolated quantum dot, and $V$ describes the coupling between 
reservoirs and quantum dot. They are given explicitly by

$$
\begin{aligned}
& H_{r e s}=\sum_{\nu \equiv \alpha \sigma \ldots} \int d \omega\left(\omega+\mu_{\alpha}\right) a_{+\nu}(\omega) a_{-\nu}(\omega) \\
& H_{S}=\sum_{s} E_{s}|s\rangle\langle s|, \\
& V=\frac{1}{2} \sum_{\eta \eta^{\prime}} \sum_{\nu \nu^{\prime}} \int d \omega \int d \omega^{\prime} \\
& g_{\eta \nu, \eta^{\prime} \nu^{\prime}}\left(\omega, \omega^{\prime}\right): a_{\eta \nu}(\omega) a_{\eta^{\prime} \nu^{\prime}}\left(\omega^{\prime}\right):
\end{aligned}
$$

Here, $a_{\eta \nu}$ are the creation $(\eta=+)$ and annihilation $(\eta=-)$ operators of the reservoirs, and $\nu$ is an index characterizing all quantum numbers of the reservoir states. In the absence of further symmetries, $\nu$ contains the reservoir index $\alpha$ and the spin quantum number $\sigma$ (for two reservoirs and spin- $\frac{1}{2}$ particles, we use the notation $\alpha \equiv L, R \equiv \pm$ and $\sigma \equiv \uparrow, \downarrow \equiv \pm) . \omega$ is the energy of the reservoir state relative to the chemical potential $\mu_{\alpha}$ of reservoir $\alpha$. The eigenstates and eigenenergies of the isolated quantum dot are denoted by $|s\rangle$ and $E_{s}$. The interaction $V$ is quadratic in the reservoir field operators, which arises from second-order processes of one electron hopping off and on the quantum dot coherently (for negative charging energies, also two electrons can hop off or on the $\left.\operatorname{dot}^{30}\right)$. This keeps the charge fixed and allows only spin and orbital fluctuations. The coupling vertex $g_{\eta \nu, \eta^{\prime} \nu^{\prime}}\left(\omega, \omega^{\prime}\right)$ is an arbitrary operator acting on the dot states. It is written in its most general form, depending on the quantum numbers and energies of the reservoir states in an arbitrary way. However, as explained in Ref. 17, the RG approach can be set up in its most convenient form if one assumes that the frequency dependence of the initial vertices is rather weak and varies on the scale of the band width $D$ of the reservoirs. Therefore, we will assume this in the following and introduce below [see Eq. (16)] a convenient cutoff function into the free reservoir Green's functions.

To achieve a more compact notation for all indices, we write $1 \equiv \eta \nu \omega$ and sum (integrate) implicitly over all indices and frequencies. The interaction is then written in the compact form

$$
V=\frac{1}{2} g_{11^{\prime}}: a_{1} a_{1^{\prime}}:
$$

: $\cdots:$ denotes normal ordering of the reservoir field operators, meaning that no contraction is allowed between reservoir field operators within the normal ordering. A contraction is defined with respect to a grand-canonical distribution of the reservoirs, given by

$$
\overparen{a_{1}} a_{1^{\prime}} \equiv\left\langle a_{1} a_{1^{\prime}}\right\rangle_{\rho_{r e s}}=\delta_{1 \overline{1}^{\prime}} f_{\alpha}(\eta \omega)
$$

$f_{\alpha}(\omega)=\left(e^{\omega / T_{\alpha}}+1\right)^{-1}=1-f_{\alpha}(-\omega)$ is the Fermi distribution function corresponding to temperature $T_{\alpha}$ (note that the chemical potential does not enter this formula since $\omega$ is measured relative to $\mu_{\alpha}$ ). Furthermore, $\delta_{11^{\prime}} \equiv \delta_{\eta \eta^{\prime}} \delta_{\nu \nu^{\prime}} \delta\left(\omega-\omega^{\prime}\right)$ is the $\delta$ function in compact notation, and $\overline{1} \equiv-\eta, \nu, \omega$. The cutoff by the band width $D$ can be introduced in many different ways into the reservoir contraction. We use a Lorentzian cutoff and replace the contraction by

$$
\overrightarrow{a_{1}} a_{1^{\prime}} \rightarrow \delta_{1 \overline{1}^{\prime}} \rho(\omega) f_{\alpha}(\eta \omega)
$$

with

$$
\rho(\omega)=\frac{D^{2}}{D^{2}+\omega^{2}} .
$$

Within the normal ordering of Eq. (14), the field operators can be arranged in an arbitrary way (up to a fermionic sign), therefore the coupling vertex can always be chosen such that antisymmetry holds,

$$
g_{11^{\prime}}=-g_{1^{\prime} 1} .
$$

Furthermore, due to the hermiticity of $V$, the vertex has the property

$$
g_{11^{\prime}}^{\dagger}=g_{\overline{1}^{\prime} \overline{1}}
$$

The particle current operator flowing from reservoir $\gamma$ to the quantum dot is defined by $I^{\gamma}=-\frac{d}{d t} N_{r e s}^{\gamma}=$ $-i\left[H, N_{\text {res }}^{\gamma}\right]$, where $N_{\text {res }}^{\gamma}$ is the particle number in reservoir $\gamma$. Using Eqs. (10) and (13), a straightforward calculation leads to

$$
I^{\gamma}=\frac{1}{2} i_{11^{\prime}}^{\gamma}: a_{1} a_{1^{\prime}}:
$$

with

$$
\begin{aligned}
& i_{11^{\prime}}^{\gamma}=-2 i c_{11^{\prime}}^{\gamma} g_{11^{\prime}} \\
& c_{11^{\prime}}^{\gamma}=-\frac{1}{2}\left(\eta \delta_{\alpha \gamma}+\eta^{\prime} \delta_{\alpha^{\prime} \gamma}\right)
\end{aligned}
$$

We are interested in the time evolution of the reduced density matrix $\rho_{S}(t)$ of the quantum dot and in the average $\left\langle I^{\gamma}\right\rangle(t)$ of the current operator. Formally, they follow from the solution of the von Neumann equation

$$
\begin{aligned}
\rho_{S}(t) & =\operatorname{Tr}_{r e s} e^{-i L\left(t-t_{0}\right)} \rho_{S}\left(t_{0}\right) \rho_{\text {res }} \\
\left\langle I^{\gamma}\right\rangle(t) & =\operatorname{Tr}_{S} \operatorname{Tr}_{r e s}\left(-i L_{I^{\gamma}}\right) e^{-i L\left(t-t_{0}\right)} \rho_{S}\left(t_{0}\right) \rho_{\text {res }}
\end{aligned}
$$

where

$$
L=[H, \cdot]_{-} \quad, \quad L_{I^{\gamma}}=\frac{i}{2}\left[I^{\gamma}, \cdot\right]_{+}
$$

are operators in Liouville space acting on usual operators in Hilbert space via the (anti)commutator $[A, B]_{ \pm}=$ $A B \pm B A$. Initially, we have assumed that the density matrix is a product of an arbitrary dot part $\rho_{S}\left(t_{0}\right)$ and a grandcanonical distribution $\rho_{\text {res }}$ for the reservoirs. It is convenient to introduce the Laplace transform

$$
\begin{aligned}
& \tilde{\rho}_{S}(E)=\int_{t_{0}}^{\infty} d t e^{i E\left(t-t_{0}\right)} \rho_{S}(t) \\
& =\operatorname{Tr}_{r e s} \frac{i}{E-L} \rho_{S}\left(t_{0}\right) \rho_{r e s} \\
& \left\langle\tilde{I^{\gamma}}\right\rangle(E)=\int_{t_{0}}^{\infty} d t e^{i E\left(t-t_{0}\right)}\left\langle I^{\gamma}\right\rangle(t) \\
& =\operatorname{Tr}_{S} \operatorname{Tr}_{r e s} L_{I^{\gamma}} \frac{1}{E-L} \rho_{S}\left(t_{0}\right) \rho_{\text {res }} \text {. }
\end{aligned}
$$




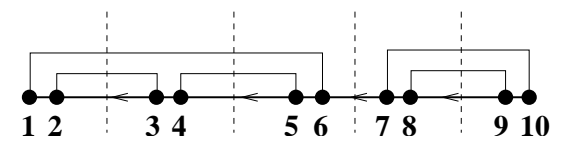

FIG. 2: Example of a diagram for the reduced density matrix of the dot. The time direction is to the left. The dots represent the interaction vertex $G$ between the local quantum system and the reservoirs. Each vertex corresponds to two adjacent dots indicating that two reservoir field operators are associated with each vertex. The horizontal lines connecting the vertices denote the free time propagation of the quantum system, leading to the resolvents $\frac{1}{E+X_{i}-L_{S}^{(0)}}$ in Laplace space. The lines connecting the dots are the reservoir contractions arising from the application of Wick's theorem. The dashed vertical lines between the vertices are auxiliary lines to determine the energy argument $X_{i}$ of the resolvents.

Perturbative expansion. The next step is to expand expressions (26) and (27) in the interacting part $L_{V}=$ $[V, \cdot]_{-}$of the Liouvillian and to integrate out the reservoir part. As outlined in detail in Ref. 17, this leads to a diagrammatic representation in Liouville space. We shortly summarize this procedure here. First, $L_{V}$ can be written in the form

$$
L_{V}=\frac{1}{2} p^{\prime} G_{11^{\prime}}^{p p^{\prime}}: J_{1}^{p} J_{1^{\prime}}^{p^{\prime}}:
$$

where $J_{1}^{p}$ is a quantum field superoperator in Liouville space for the reservoirs, defined by ( $A$ is an arbitrary reservoir operator)

$$
J_{1}^{p} A=\left\{\begin{array}{ll}
a_{1} A & \text { for } p=+ \\
A a_{1} & \text { for } p=-
\end{array} .\right.
$$

$p= \pm$ is the Keldysh index indicating whether the field operator is acting on the upper or the lower part of the Keldysh contour. $G_{11^{\prime}}^{p p^{\prime}}$ is a superoperator acting in Liouville space of the quantum dot, and is defined by ( $A$ is an arbitrary operator of the quantum dot)

$$
G_{11^{\prime}}^{p p^{\prime}} A=\delta_{p p^{\prime}}\left\{\begin{array}{cl}
g_{11^{\prime}} A & \text { for } p=+ \\
-A g_{11^{\prime}} & \text { for } p=-
\end{array} .\right.
$$

Inserting the form (28) into Eqs. (26) and (27), expanding in $L_{V}$, and shifting all reservoir field superoperators $J_{1}^{p}$ to the right, one can show that each term of perturbation theory can be written as a product of a dot part and an average over a sequence of field superoperators of the reservoirs with respect to $\rho_{\text {res }}$. Evaluating the latter with the help of Wick's theorem, one can represent each term of the Wick decomposition by a diagram, see, e.g., Fig. 2 describing a certain process for the time evolution of the reduced density matrix of the dot. Each process consists of a sequence of interaction vertices $G_{11^{\prime}}^{p p^{\prime}}$ between the dot and the reservoirs, and a free time propagation of the dot in between (leading to resolvents in Laplace space). Since the reservoirs have been integrated out, the vertices are connected by reservoir contractions (the solid lines without arrows in Fig. 21). This means that the various diagrams represent terms for the effective time evolution of the dot in the presence of dissipative reservoirs. Each diagram for the reduced density matrix has the form

$$
\begin{aligned}
& \tilde{\rho}_{S}(E) \rightarrow \\
& \frac{i}{S}(-1)^{N_{p}}\left(\prod \gamma\right) \frac{1}{E-L_{S}^{(0)}} G \frac{1}{E+X_{1}-L_{S}^{(0)}} \\
& . G \ldots G \frac{1}{E+X_{r}-L_{S}^{(0)}} G \frac{1}{E-L_{S}^{(0)}} \rho_{S}\left(t_{0}\right)
\end{aligned}
$$

where

$$
L_{S}^{(0)}=\left[H_{S}, \cdot\right]_{-}
$$

$G \equiv G_{i j}^{p_{i} p_{j}}$ indicates an interaction vertex, and $\gamma \equiv \gamma_{i j}^{p_{i} p_{j}}$ is a contraction between the reservoir field superoperators, defined by

$$
\begin{aligned}
\gamma_{11^{\prime}}^{p p^{\prime}} & =\bar{J}_{1}^{p} J_{1^{\prime}}^{p^{\prime}}=p^{\prime} \operatorname{Tr}_{r e s} J_{1}^{p} J_{1^{\prime}}^{p^{\prime}} \rho_{\text {res }} \\
& =\delta_{1 \overline{1}^{\prime}} \rho(\omega) p^{\prime} f_{\alpha}\left(\eta p^{\prime} \omega\right) .
\end{aligned}
$$

To factorize the Wick decomposition, a fermionic sign has to be assigned to each permutation of reservoir field superoperators, indicated by the sign factor $(-1)^{N_{p}}$ in Eq. (31). For each pair of vertices connected by two reservoir lines, a combinatorical factor $\frac{1}{2}$ occurs, leading to the prefactor $\frac{1}{S}$ in Eq. (31). The value of the frequencies $X_{i}$ in the resolvents between the interaction vertices is determined by the sum over all variables $x=\eta\left(\omega+\mu_{\alpha}\right)$ of those indices belonging to the reservoir lines which are crossed by a vertical line at the position of the resolvent (see the dashed lines in Fig. 2). Thereby, the index of the left vertex has to be taken of the corresponding reservoir line, e.g., the diagram of Fig. 2 is given by (the obvious dependence on the Keldysh indices has been omitted for simplicity, i.e., $\gamma_{i j} \equiv \gamma_{i j}^{p_{i} p_{j}}$ and $G_{i j} \equiv G_{i j}^{p_{i} p_{j}}$ )

$$
\begin{array}{r}
i \frac{1}{E-L_{S}^{(0)}}\left(\gamma_{16} \gamma_{23} \gamma_{45} G_{12} \Pi_{12} G_{34} \Pi_{14} G_{56}\right) \frac{1}{E-L_{S}^{(0)}} \\
\quad\left(\frac{1}{2} \gamma_{7,10} \gamma_{89} G_{78} \Pi_{78} G_{9,10}\right) \frac{1}{E-L_{S}^{(0)}} \rho_{S}\left(t_{0}\right),(34)
\end{array}
$$

where the resolvents are defined by

$$
\Pi_{1 \ldots n}=\frac{1}{E_{1 \ldots n}+\bar{\omega}_{1 \ldots n}-L_{S}^{(0)}},
$$

with

$$
\begin{aligned}
E_{1 \ldots n} & =E+\sum_{i=1}^{n} \bar{\mu}_{i}, \quad \bar{\omega}_{1 \ldots n}=\sum_{i=1}^{n} \bar{\omega}_{i} \\
\bar{\mu}_{i} & =\eta_{i} \mu_{\alpha_{i}} \quad, \quad \bar{\omega}_{i}=\eta_{i} \omega_{i} .
\end{aligned}
$$

As can be seen from example (34), each diagram consists of a sequence of irreducible blocks (where a vertical line always cuts at least one reservoir line) and free resolvents 
$1 /\left(E-L_{S}^{(0)}\right)$ in between. Similiar to Dyson equations one can formally resum this series with the result

$$
\tilde{\rho}_{S}(E)=\frac{i}{E-L_{S}^{e f f}(E)} \rho_{S}\left(t_{0}\right),
$$

with

$$
L_{S}^{e f f}(E)=L_{S}^{(0)}+\Sigma(E)
$$

where the kernel $\Sigma(E)$ contains the sum over all irreducible diagrams. A similiar procedure can be used to calculate the average (27) of the current operator with the result

$$
\begin{aligned}
\left\langle\tilde{I^{\gamma}}\right\rangle(E) & =\operatorname{Tr}_{S} \Sigma_{\gamma}(E) \frac{1}{E-L_{S}^{e f f}(E)} \rho_{S}\left(t_{0}\right) \\
& =-i \operatorname{Tr}_{S} \Sigma_{\gamma}(E) \tilde{\rho}_{S}(E)
\end{aligned}
$$

where the current kernel $\Sigma_{\gamma}(E)$ is defined similiarly to $\Sigma(E)$, but the first vertex $G$ is replaced by the current vertex $I^{\gamma}$, defined by

$$
\left(I^{\gamma}\right)_{11^{\prime}}^{p p^{\prime}}=c_{11^{\prime}}^{\gamma} \delta_{p p^{\prime}} p G_{11^{\prime}}^{p p}
$$

such that, in analogy to Eq. (28),

$$
L_{I^{\gamma}}=\frac{1}{2} p^{\prime}\left(I^{\gamma}\right)_{11^{\prime}}^{p p^{\prime}}: J_{1}^{p} J_{1^{\prime}}^{p^{\prime}}: \text {. }
$$

Using Eq. (31), a certain diagram for the kernels has to be translated according to

$$
\begin{gathered}
\left\{\begin{array}{c}
\Sigma(E) \\
\Sigma_{\gamma}(E)
\end{array}\right\} \rightarrow \frac{1}{S}(-1)^{N_{p}}\left(\prod \gamma\right)_{i r r} \\
\left\{\begin{array}{c}
G \\
I^{\gamma}
\end{array}\right\} \frac{1}{E+X_{1}-L_{S}^{(0)}} G \ldots G \frac{1}{E+X_{r}-L_{S}^{(0)}} G,
\end{gathered}
$$

where the subindex irr indicates that only irreducible diagrams are allowed where any vertical line between the vertices cuts through at least one reservoir contraction.

The stationary solutions for the reduced density matrix and the current follow from the Laplace transform by $\rho_{S}^{s t}=\lim _{E \rightarrow 0^{+}}(-i E) \tilde{\rho}_{S}(E)$ and $\left\langle I^{\gamma}\right\rangle^{s t}=$ $\lim _{E \rightarrow 0^{+}}(-i E)\left\langle\tilde{I}^{\gamma}\right\rangle(E)$, and can be calculated from

$$
\begin{aligned}
L_{S}^{e f f}\left(i 0^{+}\right) \rho_{S}^{s t} & =0 \\
\left\langle I^{\gamma}\right\rangle^{s t} & =-i \operatorname{Tr}_{S} \Sigma_{\gamma}\left(0^{+}\right) \rho_{S}^{s t}
\end{aligned}
$$

In addition to previous formulations ${ }^{17}$ of the perturbation series, we note that the diagrammatic series can be partially resummed by taking all closed subdiagrams between two fixed vertices together which contain only contractions connecting vertices between the two fixed ones. This has the effect that the resolvents in Eq. (43) are replaced by

$$
\frac{1}{E+X_{i}-L_{S}^{(0)}} \rightarrow \frac{1}{E+X_{i}-L_{S}^{e f f}\left(E+X_{i}\right)}
$$

i.e., the full effective Liouville operator occurs in the denominator. This means that Eqs. (39) and (43) turn into self-consistent equations for $L_{S}^{e f f}(E)$ for any approximation. Of course, the number of diagrams is reduced in this formulation. No diagrams are allowed anymore which contain closed subdiagrams between two vertices.

When calculating diagrams with the replacement (46), one faces the problem that the frequency integrations cannot be performed analytically because the energy dependence of the effective Liouvillian is not known. This would require the solution of a complicated selfconsistent integral equation. To avoid this, it is useful to formulate an appropriate approximation for the resolvents which can be improved systematically. To define this approximation, we write the resolvents in terms of the eigenvectors and eigenvalues of the Liouvillian $L_{S}^{e f f}(z)$

$$
\Pi(z)=\frac{1}{z-L_{S}^{e f f}(z)}=\sum_{i} \frac{1}{z-\lambda_{i}(z)} P_{i}(z)
$$

where the projectors are defined by

$$
P_{i}(z)=\left|x_{i}(z)\right\rangle\left\langle\bar{x}_{i}(z)\right|,
$$

and $\left|x_{i}(z)\right\rangle$ and $\left\langle\bar{x}_{i}(z)\right|$ are the right and left eigenvectors of $L_{S}^{e f f}(z)$

$$
\begin{aligned}
L_{S}^{e f f}(z)\left|x_{i}(z)\right\rangle & =\lambda_{i}(z)\left|x_{i}(z)\right\rangle \\
\left\langle\bar{x}_{i}(z)\right| L_{S}^{e f f}(z) & =\lambda_{i}(z)\left\langle\bar{x}_{i}(z)\right|
\end{aligned},
$$

with eigenvalues $\lambda_{i}(z)$. Assuming that $\left|x_{i}(z)\right\rangle$ and $\left\langle\bar{x}_{i}(z)\right|$ have no poles (or poles with very large negative imaginary part so that they influence only the short-time behaviour), the poles $z_{i}$ of the resolvent follow from the self-consistent equation

$$
z_{i}=\lambda_{i}\left(z_{i}\right)
$$

for all values of $i$. Expanding $\lambda_{i}(z),\left|x_{i}(z)\right\rangle$, and $\left\langle\bar{x}_{i}(z)\right|$ around $z=z_{i}$, we see that the nonanalytic part of the resolvent is given by

$$
\Pi(z) \approx \sum_{i} \frac{a_{i}}{z-z_{i}} P_{i}\left(z_{i}\right)
$$

with residua (also called $Z$ factors) given by

$$
a_{i}=\frac{1}{1-\frac{d \lambda_{i}}{d z}\left(z_{i}\right)}
$$

Equation (52) defines our approximation which is the appropriate one to describe especially line shapes at resonance (analytic parts are expected to have no special features at resonance and will only lead to an overall perturbative shift of the background). To avoid the summation index $i$, we will write the approximation in the more compact form

$$
\Pi(z) \approx \frac{\tilde{Z}}{z-\tilde{L}_{S}}
$$


where we use the convention that any function of $\tilde{Z}$ and $\tilde{L}_{S}$ is interpreted as

$$
f\left(\tilde{Z}, \tilde{L}_{S}\right) \equiv \sum_{i} f\left(\frac{1}{1-\frac{d \lambda_{i}}{d z}\left(z_{i}\right)}, z_{i}\right) P_{i}\left(z_{i}\right) .
$$

The eigenvalues $z_{i}$ can be decomposed into real and imaginary parts

$$
z_{i}=\tilde{h}_{i}-i \tilde{\Gamma}_{i} \quad, \quad \tilde{\Gamma}_{i}>0
$$

and are the poles of the original full resolvent $\Pi(z)$. According to Eq. (38), this resolvent describes the reduced density matrix in Laplace space. Therefore, the resolvent must be analytic in the upper half of the complex plane since otherwise solutions would exist in time space which are exponentially increasing and no stationary state can be reached. Thus, the negative imaginary parts $\tilde{\Gamma}_{i}$ must be strictly positive and describe the various relaxation and dephasing rates of the different modes described by the eigenvectors $\left|x_{i}\left(z_{i}\right)\right\rangle$. Correspondingly, the real parts $\tilde{h}_{i}$ describe the oscillation frequencies of the modes, e.g., the effective magnetic field for the Kondo problem.

The renormalization group treatment described in the next section can be set up within the original perturbation series (43) or the partially resummed series using the replacement (46). Since we aim at a weak coupling expansion, the partially resummed series makes only sense if the full Liouvillian is expanded in the same parameter as the renormalized vertices. Therefore, we will make use of the resummed series only at the end of the RG flow where perturbation theory in the renormalized couplings at a fixed physical cutoff scale can be used. For other problems like quantum dots in the charge fluctuation regime or systems in the strong coupling regime, it might be helpful to use the resummed series from the very beginning.

Finally, we note some useful symmetry properties for the vertices and the Liouvillian (see Ref. 17 for the proof),

$$
\begin{aligned}
\bar{G}_{12} & =-\bar{G}_{21}, \\
\bar{I}_{12}^{\gamma} & =-\bar{I}_{21}^{\gamma}, \\
\operatorname{Tr}_{S} L_{S}^{e f f}(z) & =0, \\
\operatorname{Tr}_{S} \bar{G}_{12} & =0, \\
L_{S}^{e f f}(z)^{c} & =-L_{S}^{e f f}\left(-z^{*}\right), \\
\Sigma_{\gamma}(z)^{c} & =-\Sigma_{\gamma}\left(-z^{*}\right), \\
\left(\bar{G}_{12}\right)^{c} & =-\bar{G}_{\overline{2} \overline{1}}\left(-z^{*}\right), \\
\left(\bar{I}_{12}^{\gamma}\right)^{c} & =-\bar{I}_{\overline{2} \overline{1}}^{\gamma}\left(-z^{*}\right),
\end{aligned}
$$

where

$$
\bar{G}_{11^{\prime}}=\sum_{p} G_{11^{\prime}}^{p p}, \quad \bar{I}_{11^{\prime}}^{\gamma}=\sum_{p}\left(I^{\gamma}\right)_{11^{\prime}}^{p p},
$$

and the $c$ transform $A^{c}$ of any dot operator $A$ in Liouville space is defined by

$$
\left(A^{c}\right)_{s s^{\prime}, \bar{s}^{\prime}}=A_{s^{\prime} s, \bar{s}^{\prime} \bar{s}}^{*}
$$

Properties (61) and (62) are important to show in time space that the reduced density matrix of the dot stays hermitian and the current stays real. Property (59) leads to conservation of probability, i.e., the normalization of the reduced density matrix stays constant. From this property it also follows that $L_{S}^{e f f}(z)$ has an eigenvector with zero eigenvalue:

$$
L_{S}^{e f f}(z)\left|x_{0}(z)\right\rangle=0
$$

This eigenvector corresponds to the stationary state for $z \rightarrow i 0^{+}$and depends on the physical system under consideration. In contrast, the corresponding left eigenvector is unique and, according to Eq. (59), is given by

$$
\left\langle\bar{x}_{0}(z) \mid s s^{\prime}\right\rangle=\delta_{s s^{\prime}} .
$$

As a consequence, in combination with property (60), we obtain zero if the left eigenvector for zero eigenvalue acts from the left on the vertex averaged over the Keldysh indices:

$$
\left\langle\bar{x}_{0}(z)\right| \bar{G}_{12}=0
$$

Therefore, by decomposing the vertex according to

$$
G_{11^{\prime}}^{p p}=\frac{1}{2}\left(\bar{G}_{11^{\prime}}+p \tilde{G}_{11^{\prime}}\right) \quad, \quad \tilde{G}_{11^{\prime}}=\sum_{p} p G_{11^{\prime}}^{p p}
$$

we see that the zero eigenvalue of $L_{S}^{e f f}(z)$ can only occur in the resolvents when the part $\tilde{G}_{11^{\prime}}$ of the vertex is standing right to the resolvent. Therefore, to avoid this zero eigenvalue in the RG treatment, we will first use a certain perturbative treatment to eliminate the part $\tilde{G}_{11^{\prime}}$ of the vertex from the very beginning. This is described in the next section.

\section{B. RG equations}

First RG step. The first discrete RG step consists in integrating out the symmetric part $\frac{1}{2}\left[f_{\alpha}(\omega)+f_{\alpha}(-\omega)\right]=\frac{1}{2}$ of the Fermi function in the contraction (33). This part depends only weakly on the frequency and creates no logarithmic divergencies in perturbation theory. Furthermore, as explained in detail in Ref. 17, it is the symmetric part of the Fermi function which allows the zero eigenvalue of the effective Liouvillian $L_{S}^{e f f}(E)$ to occur in the resolvents between the vertices. This part should be integrated out before starting the continuous RG in order to show that the renormalization of the vertices is cut off by relaxation and dephasing rates. To get rid of the symmetric part, one decomposes the contraction (33) according to

$$
\begin{aligned}
\gamma_{11^{\prime}}^{p p^{\prime}} & =\delta_{1 \overline{1}^{\prime}} p^{\prime} \gamma_{1}^{s}+\delta_{1 \overline{1}^{\prime}} \gamma_{1}^{a}, \\
\gamma_{1}^{s} & =\frac{1}{2} \rho(\bar{\omega}), \quad \gamma_{1}^{a}=\rho(\bar{\omega})\left[f_{\alpha}(\bar{\omega})-\frac{1}{2}\right],
\end{aligned}
$$



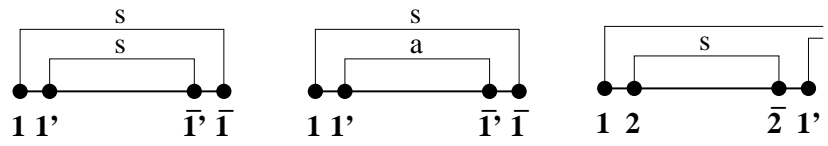

FIG. 3: The lowest order diagrams for the effective Liouvillian and the effective vertex when the symmetric part of the contraction is integrated out. $s(a)$ denotes the symmetric (antisymmetric) contraction $\gamma^{s}\left(\gamma^{a}\right)$.

with $\bar{\omega} \equiv \eta \omega$. Using this decomposition in Eq. (43), one finds that each diagram, which is irreducible with respect to the full contraction $\gamma$, decomposes into a series of blocks which are irreducible with respect to the symmetric part $\gamma^{s}$ (i.e., any vertical line hits at least one symmetric contraction) and connected to each other by antisymmetric contractions $\gamma^{a}$. The blocks which are irreducible with respect to $\gamma^{s}$ can be formally resummed into an effective Liouvillian $L^{a}(E)$ or into effective vertices $G^{a}(E)$, which obtain an additional energy variable to account for the reservoir contractions which cross over the effective quantities. The lowest-order diagrams for $L^{a}$ and $G^{a}$ are shown in Fig. 3. The first two diagrams correspond to the effective Liouvillian (no free lines) and the third one to the effective vertex (since two lines are free). Using the diagrammatic rules (43) together with Eq. (71) and the convention (36), we obtain for the first two diagrams,

$$
\gamma_{1}^{s}\left(\frac{1}{2} \gamma_{1^{\prime}}^{s}+p^{\prime} \gamma_{1^{\prime}}^{a}\right) G_{11^{\prime}}^{p p} \frac{1}{E_{11^{\prime}}+\bar{\omega}_{11^{\prime}}-L_{S}^{(0)}} G_{\overline{1}^{\prime}, \overline{1}^{\prime}}^{p^{\prime}}
$$

and for the third one (including the interchange $1 \leftrightarrow 1^{\prime}$ )

$$
p^{\prime} \gamma_{2}^{s} G_{12}^{p p} \frac{1}{E_{12}+\bar{\omega}_{12}-L_{S}^{(0)}} G_{\overline{2} 1^{\prime}}^{p^{\prime} p^{\prime}}-\left(1 \leftrightarrow 1^{\prime}\right)
$$

We use here the original perturbation series (43) so that the unperturbed Liouvillian $L_{S}^{(0)}$ occurs in the resolvents. Performing the frequency integrations and omitting terms of order $O(1 / D)$, we obtain the following perturbative result for the effective Liouvillian and the effective vertex containing the symmetric part of the contraction:

$$
\begin{aligned}
L_{S}^{a}(E)= & L_{S}+\Sigma^{a}(E), \\
\Sigma^{a}(E)= & -i \frac{\pi^{2}}{16} D \bar{G}_{11^{\prime}} \bar{G}_{\overline{1}^{\prime} \overline{1}}-\frac{\pi}{4} D \bar{G}_{11^{\prime}} \tilde{G}_{\overline{1}^{\prime} \overline{1}} \\
& +\frac{\pi^{2}}{32} \bar{G}_{11^{\prime}}\left(E_{11^{\prime}}-L_{S}^{(0)}\right) \bar{G}_{\overline{1}^{\prime} \overline{1}} \\
& -i \frac{\pi}{4} \bar{G}_{11^{\prime}}\left(E_{11^{\prime}}-L_{S}^{(0)}\right) \tilde{G}_{\overline{1}^{\prime} \overline{1}}, \\
\bar{G}_{11^{\prime}}^{a}= & \bar{G}_{11^{\prime}}-i \frac{\pi}{2}\left(\bar{G}_{12} \tilde{G}_{\overline{2} 1^{\prime}}-\bar{G}_{1^{\prime} 2} \tilde{G}_{\overline{2} 1}\right) .
\end{aligned}
$$

Analog equations hold for the effective current kernel $\Sigma_{\gamma}^{a}(E)$ and for the effective current vertex $I^{\gamma, a}$. These are obtained by replacing the first vertex $G$ by the current vertex $I^{\gamma}$ in Eqs. (74) and (75).

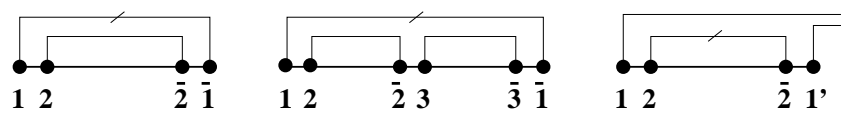

FIG. 4: RG diagrams for the renormalization of the Liouvillian in $O\left(G^{2}\right)$ and $O\left(G^{3}\right)$ (first two diagrams) and the one-loop renormalization of the vertex in $O\left(G^{2}\right)$ (last diagram). The slash indicates the contraction where the Fermi function has to be replaced by $-d \Lambda \frac{d f_{\alpha}^{\Lambda}}{d \Lambda}$.
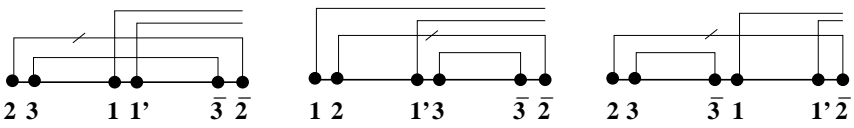

FIG. 5: RG diagrams for the renormalization of the vertex in $O\left(G^{3}\right)$ (two-loop).

After integrating out the symmetric part of the Fermi function in this way, we obtain a new diagrammatic series for the kernels analog to Eq. (43), but the Liouvillian and the vertices have to be replaced by the effective ones and the contractions between the effective vertices contain only the antisymmetric part $\gamma^{a}$. Furthermore, since the effective quantities have become energy dependent (also the effective vertex $\bar{G}^{a}$ becomes energy dependent in higher order perturbation theory), one has to replace

$$
\frac{1}{E+X_{i}-L_{S}^{(0)}} G \rightarrow \frac{1}{E+X_{i}-L_{S}^{a}\left(E+X_{i}\right)} \bar{G}^{a}\left(E+X_{i}\right)
$$

in Eq. (43). Since the antisymmetric part of the contraction (711) does not depend on the Keldysh indices, only the effective vertex $\bar{G}^{a}$ averaged over the Keldysh indices occurs in the new perturbative series. As a consequence [see Eq. (69)], the zero eigenvalue of the effective Liouvillian can no longer occur in the denominator of the resolvents.

Second RG step. The task of the second continuous $\mathrm{RG}$ procedure is to integrate out the antisymmetric part of the Fermi distribution function step by step. In each infinitesimal step, a small energy shell is integrated out and is incorporated into renormalizations of the vertices and the Liouvillian. However, instead of integrating out the energies on the real axis, it has turned out to be more efficient to integrate out the Matsubara poles of the Fermi distribution function on the imaginary axis, $\stackrel{17.28}{ }$ i.e., in each RG step one integrates out one Matsubara pole starting from high energies. To obtain a continuum version at finite temperatures, one introduces a formal cutoff dependence into the antisymmetric part of the Fermi distribution by

$$
f_{\alpha}^{\Lambda}(\omega)=-T_{\alpha} \sum_{n} \frac{1}{\omega-i \omega_{n}^{\alpha}} \theta_{T_{\alpha}}\left(\Lambda-\left|\omega_{n}^{\alpha}\right|\right),
$$

where $\omega_{n}^{\alpha}=(2 n+1) \pi T_{\alpha}$ are the Matsubara frequencies corresponding to the temperature of reservoir $\alpha$, and

$$
\theta_{T}(\omega)=\left\{\begin{array}{cl}
\theta(\omega) & \text { for }|\omega|>\pi T \\
\frac{1}{2}+\frac{\omega}{2 \pi T} & \text { for }|\omega|<\pi T
\end{array}\right.
$$


is a theta function smeared by temperature. For $\Lambda=\infty$, Eq. (76) yields the full antisymmetric part $f_{\alpha}(\omega)-\frac{1}{2}$ of the Fermi distribution. In each RG step, one reduces the cutoff $\Lambda$ by $d \Lambda$, and integrates out the infinitesimal part $f_{\alpha}^{\Lambda}-f_{\alpha}^{\Lambda-d \Lambda}=d \Lambda \frac{d f_{\alpha}^{\Lambda}}{d \Lambda}$ of the Fermi distribution. The new effective Liouvillian and the new effective vertices at scale $\Lambda-d \Lambda$,

$$
\begin{aligned}
& L_{S}^{\Lambda-d \Lambda}(E)=L_{S}^{\Lambda}(E)-d L_{S}^{\Lambda}(E), \\
& \bar{G}_{11^{\prime}}^{\Lambda-d \Lambda}(E)=\bar{G}_{11^{\prime}}^{\Lambda}(E)-d \bar{G}_{11^{\prime}}^{\Lambda}(E),
\end{aligned}
$$

can be calculated technically in the same way as for the first discrete RG step. The only difference is that an infinitesimal small part is integrated out so that the RG diagrams contain only one contraction involving the part $d \Lambda \frac{d f_{\alpha}^{\Lambda}}{d \Lambda}$. Furthermore, since the diagrams should be irreducible with respect to this part, this contraction must connect the first with the last vertex of the diagram.

Up to $O\left(G^{3}\right)$ (which we call two-loop here ${ }^{39}$ ), the RG diagrams for the Liouvillian and the vertices are shown in Figs. 4 and 5, Using the definition

$$
\gamma_{1}^{\Lambda}=\rho(\bar{\omega}) f_{\alpha}^{\Lambda}(\bar{\omega})
$$

together with the convention $L_{S} \equiv L_{S}^{\Lambda}, \bar{G}_{11^{\prime}} \equiv \bar{G}_{11^{\prime}}^{\Lambda}$ and

$$
\Pi_{1 \ldots n}=\frac{1}{E_{1 \ldots n}+\bar{\omega}_{1 \ldots n}-L_{S}\left(E_{1 \ldots n}+\bar{\omega}_{1 \ldots n}\right)},
$$

we obtain the following RG equations:

$$
\begin{aligned}
\frac{d L_{S}(E)}{d \Lambda}= & -\frac{d \gamma_{1}^{\Lambda}}{d \Lambda} \gamma_{2}^{\Lambda} \bar{G}_{12}(E) \Pi_{12} \bar{G}_{\overline{2} \overline{1}}\left(E_{12}+\bar{\omega}_{12}\right) \\
& -\frac{d \gamma_{1}^{\Lambda}}{d \Lambda} \gamma_{2}^{\Lambda} \gamma_{3}^{\Lambda} \bar{G}_{12}(E) \Pi_{12} \bar{G}_{\overline{2} 3}\left(E_{12}+\bar{\omega}_{12}\right) \Pi_{13} \bar{G}_{\overline{3} \overline{1}}\left(E_{13}+\bar{\omega}_{13}\right)
\end{aligned}
$$

for the Liouvillian, and

$$
\begin{aligned}
& \frac{d \bar{G}_{11^{\prime}}(E)}{d \Lambda}=-\left\{\frac{d \gamma_{2}^{\Lambda}}{d \Lambda} \bar{G}_{12}(E) \Pi_{12} \bar{G}_{\overline{2} 1^{\prime}}\left(E_{12}+\bar{\omega}_{12}\right)-\left(1 \leftrightarrow 1^{\prime}\right)\right\} \\
& \quad-\frac{d \gamma_{2}^{\Lambda}}{d \Lambda} \gamma_{3}^{\Lambda} \bar{G}_{23}(E) \Pi_{23} \bar{G}_{11^{\prime}}\left(E_{23}+\bar{\omega}_{23}\right) \Pi_{11^{\prime} 23} \bar{G}_{\overline{3} \overline{2}}\left(E_{11^{\prime} 23}+\bar{\omega}_{11^{\prime} 23}\right) \\
& \quad+\left\{\frac{d \gamma_{2}^{\Lambda}}{d \Lambda} \gamma_{3}^{\Lambda} \bar{G}_{12}(E) \Pi_{12} \bar{G}_{1^{\prime} 3}\left(E_{12}+\bar{\omega}_{12}\right) \Pi_{11^{\prime} 23} \bar{G}_{\overline{3} \overline{2}}\left(E_{11^{\prime} 23}+\bar{\omega}_{11^{\prime} 23}\right)-\left(1 \leftrightarrow 1^{\prime}\right)\right\} \\
& \quad-\left\{\frac{d \gamma_{2}^{\Lambda}}{d \Lambda} \gamma_{3}^{\Lambda} \bar{G}_{23}(E) \Pi_{23} \bar{G}_{\overline{3} 1}\left(E_{23}+\bar{\omega}_{23}\right) \Pi_{12} \bar{G}_{1^{\prime} \overline{2}}\left(E_{12}+\bar{\omega}_{12}\right)-\left(1 \leftrightarrow 1^{\prime}\right)\right\}
\end{aligned}
$$

for the vertex. Similiar RG equations hold for the current kernel $\Sigma_{\gamma}(E)$ and the current vertex $I_{11^{\prime}}^{\gamma}(E)$ by replacing the first vertex in all terms on the r.h.s. of the RG equation by the current vertex. The initial conditions of the RG equations are given by Eqs. (73)-(75) from the first discrete RG step. Since $\gamma_{1}^{\Lambda=0}=0$, the final solution at $\Lambda=0$ provides the result for the effective Liouvillian and the current kernel

$$
\begin{aligned}
L_{S}^{e f f}(E) & =\left.L_{S}(E)\right|_{\Lambda=0}, \\
\Sigma_{\gamma}(E) & =\left.\Sigma_{\gamma}(E)\right|_{\Lambda=0},
\end{aligned}
$$

from which the reduced density matrix and the current can be calculated in Laplace space via Eqs. (38) and (40).

We note that one can stop at each step of the RG and use the perturbative series (43) with the contractions $\gamma_{1}^{\Lambda}$ at scale $\Lambda$ together with the replacement

$$
\frac{1}{E+X_{i}-L_{S}^{(0)}} G \rightarrow \frac{1}{E+X_{i}-L_{S}^{\Lambda}\left(E+X_{i}\right)} \bar{G}^{\Lambda}\left(E+X_{i}\right)
$$

for the resolvents and the vertices, where $L_{S}^{\Lambda}(E)$ and $\bar{G}^{\Lambda}(E)$ are the renormalized quantities at scale $\Lambda$ [in higher order in the coupling, also vertices $\bar{G}_{1 \ldots n}^{\Lambda}(E)$ with more than two indices can be generated]. If the exact $\mathrm{RG}$ equations in all orders are used, this perturbative series gives the full kernels at each scale $\Lambda$. Therefore, it is possible at each step of the RG to resum all closed subdiagrams between two vertices, leading to the replacement

$$
\frac{1}{E+X_{i}-L_{S}^{\Lambda}\left(E+X_{i}\right)} \rightarrow \frac{1}{E+X_{i}-L_{S}^{e f f}\left(E+X_{i}\right)},
$$

where $L_{S}^{e f f}(E)$ is the full effective Liouvillian at the end of the RG flow at scale $\Lambda=0$. Using the perturbative series in a certain approximation, one can set up a selfconsistent equation for $L_{S}^{e f f}(E)$ at each step of the RG. However, this is only possible if the perturbation theory in the renormalized coupling is well defined. We will see that this is only possible at a certain scale $\Lambda_{c}$ where some physical cutoff scale is reached, see Sec. IIC Up to 
this scale, we will always use the renormalized Liouvillian $L_{S}^{\Lambda}(E)$ in the denominator of the resolvents.

$R G$ in Matsubara space. Using the fact that the resolvents and the vertices on the r.h.s. of the RG equations are analytic functions in all frequencies $\bar{\omega}_{i}$ in the upper half of the complex plane, all frequency integrations can be calculated analytically by closing the contour in the upper half of the complex plane. The only poles occurring there are the poles of the contractions and their derivatives, given by

$$
\begin{aligned}
\gamma_{1}^{\Lambda} & =-\rho(\bar{\omega}) T_{\alpha} \sum_{n} \frac{1}{\bar{\omega}-i \omega_{n}^{\alpha}} \theta_{T_{\alpha}}\left(\Lambda-\left|\omega_{n}^{\alpha}\right|\right),( \\
\frac{d}{d \Lambda} \gamma_{1}^{\Lambda} & =-\rho(\bar{\omega}) \frac{1}{2 \pi}\left(\frac{1}{\bar{\omega}-i \Lambda_{T_{\alpha}}}+\frac{1}{\omega+i \Lambda_{T_{\alpha}}}\right)
\end{aligned}
$$

$\Lambda_{T_{\alpha}}$ denotes the Matsubara frequency $\omega_{n}^{\alpha}$ which lies closest to the cutoff $\Lambda$. After performing the integration we find that, due to the presence of the cutoff function $\rho(\bar{\omega})=\frac{D^{2}}{D^{2}+\bar{\omega}^{2}}$, the r.h.s. of the RG equations gives a negligible contribution for $\Lambda \gg D$. Therefore, we can start the RG at $\Lambda_{0} \sim D$ and omit the cutoff function $\rho(\bar{\omega})$ (finally, the precise ratio between $\Lambda_{0}$ and $D$ is determined such that no linear terms in $D$ are generated, see below). As a consequence, only the Matsubara poles of the Fermi function in the upper half of the complex plane will contribute to the frequency integrations and all real frequencies are replaced by Matsubara frequencies. From now on, we write the frequency dependence explicitly and define the analytic continuation of the Liouvillian and the vertices in imaginary frequency space by

$$
\begin{aligned}
L_{S}(E, \omega) & =L_{S}(E+i \omega) \\
\bar{G}_{\eta_{1} \nu_{1}, \eta_{2} \nu_{2}}\left(E, \omega, \omega_{1}, \omega_{2}\right) & =\left.\bar{G}_{12}(E+i \omega)\right|_{\bar{\omega}_{i} \rightarrow i \omega_{i}}
\end{aligned}
$$

where $\omega \equiv \omega_{n}^{\alpha}, \omega_{i} \equiv \omega_{n_{i}}^{\alpha_{i}}$ correspond to Matsubara frequencies. With the definition

$$
\Pi(E, \omega)=\frac{1}{E+i \omega-L_{S}(E, \omega)},
$$

the RG equations in Matsubara space can be written as

$$
\begin{aligned}
\frac{d L_{S}(E, \omega)}{d \Lambda}= & \bar{G}_{12}\left(E, \omega, \Lambda_{T_{\alpha_{1}}}, \omega_{2}\right) \Pi\left(E_{12}, \Lambda_{T_{\alpha_{1}}}+\omega+\omega_{2}\right) \bar{G}_{\overline{2} \overline{1}}\left(E_{12}, \Lambda_{T_{\alpha_{1}}}+\omega+\omega_{2},-\omega_{2},-\Lambda_{T_{\alpha_{1}}}\right) \\
& -i \bar{G}_{12} \Pi\left(E_{12}, \Lambda_{T_{\alpha_{1}}}+\omega+\omega_{2}\right) \bar{G}_{\overline{2} 3} \Pi\left(E_{13}, \Lambda_{T_{\alpha_{1}}}+\omega+\omega_{3}\right) \bar{G}_{\overline{3} \overline{1}} \quad, \\
\frac{d \bar{G}_{11^{\prime}}\left(E, \omega, \omega_{1}, \omega_{1}^{\prime}\right)}{d \Lambda}= & i\left\{\bar{G}_{12}\left(E, \omega, \omega_{1}, \Lambda_{T_{\alpha_{2}}}\right) \Pi\left(E_{12}, \Lambda_{T_{\alpha_{2}}}+\omega+\omega_{1}\right) \bar{G}_{\overline{2} 1^{\prime}}\left(E_{12}, \Lambda_{T_{\alpha_{2}}}+\omega+\omega_{1},-\Lambda_{T_{\alpha_{2}}}, \omega_{1}^{\prime}\right)-\left(1 \leftrightarrow 1^{\prime}\right)\right\} \\
+ & \bar{G}_{23} \Pi\left(E_{23}, \Lambda_{T_{\alpha_{2}}}+\omega+\omega_{3}\right) \bar{G}_{11^{\prime}} \Pi\left(E_{11^{\prime} 23}, \Lambda_{T_{\alpha_{2}}}+\omega+\omega_{1}+\omega_{1}^{\prime}+\omega_{3}\right) \bar{G}_{\overline{3} \overline{2}} \\
- & \left\{\bar{G}_{12} \Pi\left(E_{12}, \Lambda_{T_{\alpha_{2}}}+\omega+\omega_{1}\right) \bar{G}_{1^{\prime} 3} \Pi\left(E_{11^{\prime} 23}, \Lambda_{T_{\alpha_{2}}}+\omega+\omega_{1}+\omega_{1}^{\prime}+\omega_{3}\right) \bar{G}_{\overline{3} \overline{2}}-\left(1 \leftrightarrow 1^{\prime}\right)\right\} \\
+ & \left\{\bar{G}_{23} \Pi\left(E_{23}, \Lambda_{T_{\alpha_{2}}}+\omega+\omega_{3}\right) \bar{G}_{\overline{3} 1} \Pi\left(E_{12}, \Lambda_{T_{\alpha_{2}}}+\omega+\omega_{1}\right) \bar{G}_{1^{\prime} \overline{2}}-\left(1 \leftrightarrow 1^{\prime}\right)\right\} .
\end{aligned}
$$

In these equations, the index $1 \equiv \eta \nu$ includes no longer the frequency variable, and we implicitly sum over all indices and Matsubara frequencies on the r.h.s. of the RG equations which do not occur on the left hand side (l.h.s.). Only positive Matsubara frequencies smaller than the cutoff $\Lambda$ are allowed and each sum has to be written as

$$
2 \pi T_{\alpha} \sum_{n} \theta_{T_{\alpha}}\left(\Lambda-\omega_{n}^{\alpha}\right) \theta\left(\omega_{n}^{\alpha}\right)
$$

which reduces to an integral $\int_{0}^{\Lambda} d \omega$ for zero temperature. The frequency arguments of the vertices in the terms of $O\left(G^{3}\right)$ in Eqs. (92) and (93) have been omitted since they are not needed for the weak coupling analysis up to two-loop order, see below.

The RG equations in Matsubara space are the final result of this section and are the starting point for the analytical solution in the weak coupling regime presented in the next section. Similiar RG equations hold for the current kernel and the current vertex in Matsubara space by replacing the first vertex in all terms on the r.h.s. by the current vertex. Using Eqs. (84), (85), and (89), the effective Liouvillian and the current kernel follow from

$$
\begin{aligned}
L_{S}^{e f f}(E) & =\left.L_{S}(E, \omega=0)\right|_{\Lambda=0} \quad, \\
\Sigma_{\gamma}(E) & =\left.\Sigma_{\gamma}(E, \omega=0)\right|_{\Lambda=0}
\end{aligned} .
$$

Finally, we note that all symmetry properties stated in Eqs. (57) - 64) are preserved under the RG flow (see Ref. 17 for the proof),

$$
\begin{aligned}
\bar{G}_{12}\left(E, \omega, \omega_{1}, \omega_{2}\right) & =-\bar{G}_{21}\left(E, \omega, \omega_{2}, \omega_{1}\right), \\
\bar{I}_{12}^{\gamma}\left(E, \omega, \omega_{1}, \omega_{2}\right) & =-\bar{I}_{21}^{\gamma}\left(E, \omega, \omega_{2}, \omega_{1}\right), \\
\operatorname{Tr}_{S} L_{S}(E, \omega) & =0 \\
\operatorname{Tr}_{S} \bar{G}_{12}\left(E, \omega, \omega_{1}, \omega_{2}\right) & =0 \\
L_{S}(E, \omega)^{c} & =-L_{S}(-E, \omega), \\
\Sigma_{\gamma}(E, \omega)^{c} & =-\Sigma_{\gamma}(-E, \omega), \\
\bar{G}_{12}\left(E, \omega, \omega_{1}, \omega_{2}\right)^{c} & =-\bar{G}_{\overline{2} \overline{1}}\left(-E, \omega, \omega_{2}, \omega_{1}\right), \\
\bar{I}_{12}^{\gamma}\left(E, \omega, \omega_{1}, \omega_{2}\right)^{c} & =-\bar{I}_{\overline{2} \overline{1}}^{\gamma}\left(-E, \omega, \omega_{2}, \omega_{1}\right),
\end{aligned}
$$


where all energy variables are real.

Similiar to the discussion at the end of Sec. II A properties (99) and (100) have the consequence that we obtain zero if the left eigenvector $\left\langle\bar{x}_{0}^{\Lambda}(E, \omega)\right|$ of the effective Liouvillian $L_{S}^{\Lambda}(E, \omega)$ for zero eigenvalue acts from the left on the vertex

$$
\left\langle\bar{x}_{0}(E, \omega)\right| \bar{G}_{12}=0
$$

compare with Eq. (69). Therefore, it is not allowed that the zero eigenvalue of $L_{S}^{\Lambda}(E, \omega)$ occurs in the resolvents on the r.h.s. of the RG equations, proving that the $\mathrm{RG}$ is always cut off by relaxation and dephasing rates (this property holds in all orders of perturbation theory and within all truncation schemes). As we will see in the next section, this property is essential to prove that a systematic weak coupling analysis can be carried out in the generic case.

\section{Two-loop analysis}

In this section, we solve the two-loop RG equations (92) and (93) analytically in the weak coupling regime up to two-loop order. Weak coupling is defined by the condition that the renormalized vertices $\bar{G}_{12}\left(E, \omega, \omega_{1}, \omega_{2}\right)$ stay small compared to one throughout the RG flow so that a systematic expansion is possible on the r.h.s. of the $R G$ equations. This condition is fulfilled if the various cutoff scales occurring in the resolvents $\Pi(E, \omega)$ are larger than the energy scale $T_{K}$ at which the vertices would diverge in the absence of any cutoff scales (the so-called Kondo temperature for the Kondo model). From the form of the RG equations, we see that the resolvents at scale $\Lambda$ have the form

$$
\frac{1}{z-L_{S}^{\Lambda}(z)}
$$

with

$$
z=E_{1 \ldots n}+i \omega+i\left(\Lambda_{T_{\alpha_{1}}}+\omega_{2}+\cdots+\omega_{n}\right)
$$

and positive Matsubara frequencies $0<\omega_{k}<\Lambda$ for $k=2, \ldots, n$. Here, $E+i \omega$ is the original Laplace variable at which we want to calculate the final effective Liouvillian $L_{S}^{e f f}(E+i \omega)$, and the index 1 corresponds to the contraction connecting the first with the last vertex of the RG diagram (where the Matsubara frequency is replaced by the cutoff $\Lambda_{T_{\alpha}}$ ).

Expanding the resolvent (106) around its poles analog to the discussion at the end of Sec. IIA, we arrive at the approximation (54) which contains the most important terms leading to logarithmic enhancements

$$
\Pi^{\Lambda}(z) \approx \frac{\tilde{Z}^{\Lambda}}{z-\tilde{L}_{S}^{\Lambda}}=\sum_{i} \frac{a_{i}^{\Lambda}}{z-z_{i}^{\Lambda}}\left|x_{i}^{\Lambda}\left(z_{i}^{\Lambda}\right)\right\rangle\left\langle\bar{x}_{i}^{\Lambda}\left(z_{i}^{\Lambda}\right)\right|
$$

with

$$
\begin{aligned}
& \frac{1}{z-z_{i}^{\Lambda}}= \\
= & \frac{1}{i \Lambda_{T_{\alpha_{1}}}+E_{1 \ldots n}-\tilde{h}_{i}^{\Lambda}+i\left(\omega+\omega_{2}+\cdots+\omega_{n}\right)+i \tilde{\Gamma}_{i}^{\Lambda}},
\end{aligned}
$$

where $z_{i}^{\Lambda}=\tilde{h}_{i}^{\Lambda}-i \tilde{\Gamma}_{i}^{\Lambda}, \tilde{\Gamma}_{i}^{\Lambda}>0$, denote the positions of the nonzero poles of the resolvent (we assume single poles here, but the following discussion holds also for other cases; note that the zero pole of the stationary solution can not occur in the resolvent as discussed at the end of the last section). Since all Matsubara frequencies and the relaxation/dephasing rates are positive, we see that the resolvents can not become large. Using $0<\omega_{k}<\Lambda$ for $k=2, \ldots, n$, and setting $\omega=0$, we find that the resolvent is cut off at the scale

$$
\Lambda \sim \max \left\{T_{\alpha},\left|E_{1 \ldots n}-\tilde{h}_{i}^{\Lambda}\right|, \tilde{\Gamma}_{i}^{\Lambda}\right\}
$$

where the maximum is taken over all values of the occurring indices. Here, temperature is a trivial cutoff parameter, because, for $\Lambda<2 \pi T_{\alpha}$, the sum (94) over the Matsubara frequencies for reservoir $\alpha$ reduces to one term $n=0$ and the cutoff $\Lambda_{T_{\alpha}}=\pi T_{\alpha}$ becomes independent of $\Lambda$. Therefore, temperature is a unique cutoff for all terms on the r.h.s. of the RG equations, like in equilibrium problems. This trivial cutoff is set to zero in the following, i.e., $T_{\alpha}=0$, and we discuss only the nontrivial dependence on the other cutoff scales. The minimal cutoff scale occurs for $E_{1 \ldots n}-\tilde{h}_{i}^{\Lambda}=0$ and is given by the relaxation or dephasing rates $\tilde{\Gamma}_{i}^{\Lambda}$. These points define the positions of resonances where renormalization-groupinduced logarithmic enhancements or suppressions have to be expected. However, as we will show in the following, these logarithmic terms can be calculated systematically by perturbation theory in the renormalized couplings, provided that the weak coupling condition

$$
\Lambda_{c} \equiv \max \left\{|E|,\left|\mu_{\alpha}\right|,\left|\tilde{h}_{i}\right|\right\} \gg T_{K}
$$

is fulfilled, where by convention $\tilde{h}_{i} \equiv \tilde{h}_{i}^{\Lambda=0}$ denotes the final (physical) renormalized oscillation frequency at scale $\Lambda=0$ (we will see that the difference between $\tilde{h}_{i}^{\Lambda}$ and $\tilde{h}_{i}^{\Lambda=0}$ is proportional to the final renormalized coupling, i.e., only a small perturbative correction). $\Lambda_{c}$ is an important energy scale separating two energy regions where the RG equations are solved in a different way. It is given by the maximum of the Laplace variable $E$, the chemical potentials of the reseroirs (giving some voltage $V$ ), and the oscillation frequencies $\tilde{h}_{i}$ of the different physical modes (e.g., the renormalized magnetic field in the Kondo problem). This is roughly the maximum value the various cutoff scales $\left|E_{1 \ldots n}-\tilde{h}_{i}^{\Lambda}\right|=\left|E+\sum_{k} \eta_{k} \mu_{\alpha_{k}}-\tilde{h}_{i}^{\Lambda}\right|$ of the resolvents can take, see Eq. (110). Thus, for $\Lambda>\Lambda_{c} \gg T_{K}$, the cutoff scales do not play an important role and we get $J_{\Lambda} \ll 1$, where $J_{\Lambda}$ is the order of magnitude of the vertex at scale $\Lambda$. Since $\Lambda$ is the relevant 
energy scale in this regime, the order of magnitude of the relaxation/dephasing rates is given by $\tilde{\Gamma}_{i}^{\Lambda} \sim \Lambda J_{\Lambda}^{2} \ll \Lambda$ (note that the RG for the Liouvillian starts in second order in $J$ ). Therefore, the relaxation and dephasing rates are small perturbative corrections in the denominators of the resolvents and do not lead to any cutoff of the RG in the regime $\Lambda>\Lambda_{c}$. Since all vertices are small, we can systematically truncate the hierarchy of RG equations and expand the solution systematically around the poor man scaling solution (i.e., the lowest-order solution for the vertex in the absence of any cutoff scales $\left.\Lambda_{c}=T_{\alpha}=0\right)$. This gives a certain initial condition for the RG at $\Lambda=\Lambda_{c}$ presented as a power series in $J_{c}=J_{\Lambda=\Lambda_{c}}$.

In the second regime $0<\Lambda<\Lambda_{c}$, the $\mathrm{RG}$ for $\tilde{\Gamma}_{i}^{\Lambda}$ is very weak and will be roughly cut off by $\Lambda_{c}$. The reason is that there are many terms on the r.h.s. of the RG equation involving different cutoff scales $\left|E_{1 \ldots n}-\tilde{h}_{i}^{\Lambda}\right|$, but usually one of them will be given by $\Lambda_{c}$ already in second order in $J$. In this case (see a comment below for the other cases), we get $\tilde{\Gamma}_{i}^{\Lambda} \sim \Lambda_{c} J_{c}^{2}$ for all $0<\Lambda<\Lambda_{c}$ because $\tilde{\Gamma}_{i}^{\Lambda}$ becomes smaller for decreasing $\Lambda$. This means that even at resonance, the minimal cutoff scale is given by $\Gamma \sim \Lambda_{c} J_{c}^{2}$. Therefore, by expanding the solution for the vertices systematically in $J_{c}$, we get in the worst case at resonance a series of the schematic form

$$
J_{\Lambda} \sim J_{c}\left(1+J_{c} \ln \frac{\Lambda_{c}}{|\Lambda+i \Gamma|}+\ldots\right) .
$$

For $\Lambda \rightarrow 0$, the logarithmic term becomes maximally of the order $\sim J_{c} \ln J_{c} \ll 1$, which is a perturbative correction in the weak coupling case $J_{c} \ll 1$. Therefore, under condition (111), we stay in the weak-coupling regime and the $\mathrm{RG}$ equations can be solved perturbatively in $J_{c}$ in the whole regime $0<\Lambda<\Lambda_{c}$. We note that this fact relies essentially on the condition that all resolvents on the r.h.s. of the $\mathrm{RG}$ equations contain some relaxation/dephasing rate $\tilde{\Gamma}_{i}^{\Lambda}$. As explained at the end of the last section, our RG approach gives this property in the generic case in all orders of $J$ by construction.

In case that the RG equation for $\tilde{\Gamma}_{i}^{\Lambda}$ contains a smaller cutoff scale $\Lambda_{c}^{\prime} \ll \Lambda_{c}$ in second order in $J$, we expect $\tilde{\Gamma}_{i}^{\Lambda} \sim \Lambda_{c}^{\prime}\left(J_{c}^{\prime}\right)^{2}$ for $\Lambda<\Lambda_{c}^{\prime}$, with $J_{c}^{\prime}=J_{\Lambda=\Lambda_{c}^{\prime}}$. In this case, the logarithmic term in Eq. (112) leads to contributions $\sim J_{c} \ln \frac{\Lambda_{c}}{\Lambda_{c}^{\prime}}$ for $\Lambda \rightarrow 0$, giving rise to additional enhancements and sharper features at resonance. However, even for $\Lambda_{c}^{\prime} \rightarrow 0$, there is no divergence since the cutoff scale $\Lambda_{c}$ will certainly occur in some higher order term on the r.h.s. of the RG equation for $\tilde{\Gamma}_{i}^{\Lambda}$. Thus, the minimal cutoff scale will be of order $\sim \Lambda_{c} J_{c}^{k}$ with $k>2$. This gives a maximal value $\sim k J_{c} \ln J_{c}$ for the logarithm which is again a perturbative correction just enhanced by a factor of $k$. This shows that the height of logarithmic enhancements at resonance are expected to be increasable only by factors of $O(1)$, but the sharpness of features at resonance (which are controlled by $\tilde{\Gamma}_{i}$ ) can become orders of magnitude smaller.
As a consequence, we have seen that for $0<\Lambda<\Lambda_{c}$, we can perform a perturbation theory in $J_{c}$, which is the order of the vertex at scale $\Lambda_{c}$. This means that we can equivalently stop the $\mathrm{RG}$ at $\Lambda=\Lambda_{c}$ and use the perturbative series (43) with the contraction $\gamma_{1}^{\Lambda_{c}}$ at scale $\Lambda_{c}$ together with the replacement

$$
\begin{aligned}
& \frac{1}{E+X_{i}-L_{S}^{(0)}} G \rightarrow \\
& \quad \rightarrow \frac{1}{E+X_{i}-L_{S}^{\Lambda_{c}}\left(E+X_{i}\right)} \bar{G}^{\Lambda_{c}}\left(E+X_{i}\right)
\end{aligned}
$$

for the resolvents and the vertices. In contrast to the perturbative series at scales $\Lambda \gg \Lambda_{c}$, this perturbative series at scale $\Lambda_{c}$ is well defined and can be used alternatively to the RG approach. Furthermore, as explained in Sec. IIA, the perturbation series can be partially resummed, leading to the replacement

$$
\frac{1}{E+X_{i}-L_{S}^{\Lambda_{c}}\left(E+X_{i}\right)} \rightarrow \frac{1}{E+X_{i}-L_{S}^{e f f}\left(E+X_{i}\right)},
$$

i.e., the final full effective Liouvillian can be written in the denominator. This series has the advantage that the oscillation frequencies $\tilde{h}_{i}$, defining the resonance positions

$$
E_{1 \ldots n}=E+\sum_{k} \eta_{k} \mu_{\alpha_{k}}=\tilde{h}_{i}
$$

and the relaxation/dephasing rates $\tilde{\Gamma}_{i}$, cutting off the logarithmic enhancements at resonance, are the final physical ones at scale $\Lambda=0$. This is expected on physical grounds and leaves no question open what the precise prefactor of these energy scales is. Using the replacement (113), one can either write down directly the perturbative series or one can use the RG equations [again using the replacement (113) to define the resolvents] and solve them perturbatively in $J_{c}$. Both approaches give the same because the RG equations are formally exact.

Having shown that a weak coupling analysis is well defined for all cutoff scales under the condition (111), we proceed to show analytically the perturbative solution of the $\mathrm{RG}$ equations in all details for the two regimes $\Lambda>\Lambda_{c}$ and $0<\Lambda<\Lambda_{c}$ in the generic case.

\section{RG above $\Lambda_{c}$}

Lowest order. For $\Lambda>\Lambda_{c}$, we define the reference solution for the vertex by considering only the first term on the r.h.s. of RG equation (93) with $\Pi\left(E_{12}, \Lambda+\omega+\right.$ $\left.\omega_{1}\right) \rightarrow \frac{1}{i \Lambda}$, i.e., by setting all frequencies to zero and omitting the cutoff scales from $E, \bar{\mu}_{12}$, and $L_{S}(E)$. This defines the leading order RG equations

$$
\begin{aligned}
\frac{d \bar{G}_{11^{\prime}}^{(1)}}{d \Lambda} & =\frac{1}{\Lambda}\left\{\bar{G}_{12}^{(1)} \bar{G}_{\overline{2} 1^{\prime}}^{(1)}-\left(1 \leftrightarrow 1^{\prime}\right)\right\}, \\
\frac{d \bar{I}_{11^{\prime}}^{\gamma(1)}}{d \Lambda} & =\frac{1}{\Lambda}\left\{\bar{I}_{12}^{\gamma(1)} \bar{G}_{\overline{2} 1^{\prime}}^{(1)}-\left(1 \leftrightarrow 1^{\prime}\right)\right\} .
\end{aligned}
$$


The initial condition for these RG equations is the bare vertex. The order of magnitude of the leading order solution is denoted by the dimensionless parameter $J \sim \bar{G}_{12}^{(1)}$. The connection to conventional poor man scaling is established by recognizing that the leading order vertices have the same form in Liouville space as the original vertices, given by Eqs. (70), (30), and (41), i.e., one can prove that 17

$$
\begin{aligned}
\bar{G}_{12}^{(1)} & =\left[g_{12}, \cdot\right]_{-}, \\
\tilde{G}_{12}^{(1)} & =\left[g_{12}, \cdot\right]_{+}, \\
\bar{I}_{12}^{\gamma(1)} & =c_{12}^{\gamma} \tilde{G}_{12}^{(1)},
\end{aligned}
$$

with

$$
\frac{d g_{11^{\prime}}}{d \Lambda}=\frac{1}{\Lambda}\left\{g_{12} g_{\overline{2} 1^{\prime}}-\left(1 \leftrightarrow 1^{\prime}\right)\right\} \quad .
$$

Thereby, the form (119) of the current vertex can only be proven if one takes the $\operatorname{Tr}_{S}$ over the local quantum system, i.e., it holds only for the combination $\operatorname{Tr}_{S} \bar{I}_{12}^{\gamma(1)}$. Implicitly, for all following equations, we will always consider this combination for the current vertex because this is finally needed for the calculation of the average of the current. Equation (120) is the usual poor man scaling equation which can also be derived on a pure Hamiltonian level by, e.g., leaving the $t$ matrix invariant. $\frac{1}{1}$

Next we set up the lowest order RG equation for the Liouvillian $L_{S}(E, \omega)$ by considering the first term on the r.h.s. of Eq. (92) and replacing the vertices by the leading order ones. Furthermore, we replace $L_{S}(E, \omega)$ by $L_{S}^{(0)}$ in the resolvent. This gives

$$
\frac{d L_{S}(E, \omega)}{d \Lambda} \approx-i \bar{G}_{12}^{(1)} \mathcal{K}_{\Lambda}\left(E_{12}+i \omega-L_{S}^{(0)}\right) \bar{G}_{\overline{2} \overline{1}}^{(1)}
$$

with

$$
\mathcal{K}_{\Lambda}(z)=\ln \left(\frac{2 \Lambda-i z}{\Lambda-i z}\right)
$$

To extract the lowest-order term $L_{S}^{(1)} \sim J$ from this equation we treat the terms $\sim z / \Lambda$ of $\mathcal{K}_{\Lambda}(z)$ separately by the decomposition

$$
\mathcal{K}_{\Lambda}(z)=\tilde{\mathcal{K}}_{\Lambda}(z)+\frac{i z}{2 \Lambda}
$$

so that $\tilde{\mathcal{K}}_{\Lambda}(z)$ is integrated by the function

$$
\begin{aligned}
\tilde{\mathcal{K}}_{\Lambda}(z)= & \frac{d}{d \Lambda} \tilde{F}_{\Lambda}(z), \\
\tilde{F}_{\Lambda}(z)= & \Lambda \ln \left(\frac{2 \Lambda-i z}{\Lambda-i z}\right) \\
& -\frac{i z}{2}\left(\ln \frac{(2 \Lambda-i z) \Lambda}{2(\Lambda-i z)^{2}}+1\right),
\end{aligned}
$$

with the following asymptotic behavior:

$$
\tilde{F}_{\Lambda}(z) \rightarrow \Lambda\left[\ln 2+O\left(\frac{z}{\Lambda}\right)^{2}\right] \quad \text { for } \Lambda \gg|z| .
$$

Using Eq. (121), the second term on the r.h.s. of Eq. (123) leads to the following RG equation for the Liouvillian in leading order:

$$
\frac{d L_{S}^{(1)}(E, \omega)}{d \Lambda}=\frac{1}{2 \Lambda} \bar{G}_{12}^{(1)}\left(E_{12}+i \omega-L_{S}^{(0)}\right) \bar{G}_{\overline{2} \overline{1}}^{(1)}
$$

with the initial condition

$$
\left.L_{S}^{(1)}(E, \omega)\right|_{\Lambda=\Lambda_{0}}=0 .
$$

When integrated, we obtain a contribution $L_{S}^{(1)}(E, \omega) \sim$ $J$, i.e., one power less than expected due to the factor $\frac{1}{\Lambda}$ [compare with RG equation (115), where the same happens]. In contrast, the contributions from $\tilde{\mathcal{K}}_{\Lambda}(z)$ are of second and third order in $J$, as will be discussed below. We write

$$
L_{S}^{(1)}(E, \omega)=L_{S}^{(1)}-(E+i \omega) Z^{(1)}
$$

with $L_{S}^{(1)} \equiv L_{S}^{(1)}(E=0, \omega=0)$, and

$$
\begin{aligned}
\frac{d L_{S}^{(1)}}{d \Lambda} & =\frac{1}{2 \Lambda} \bar{G}_{12}^{(1)}\left(\bar{\mu}_{12}-L_{S}^{(0)}\right) \bar{G}_{\overline{2} \overline{1}}^{(1)}, \\
\frac{d Z^{(1)}}{d \Lambda} & =-\frac{1}{2 \Lambda} \bar{G}_{12}^{(1)} \bar{G}_{\overline{2} \overline{1}}^{(1)} .
\end{aligned}
$$

We note that $\frac{1}{1+Z^{(1)}}$ can be interpreted as the $Z$ factor in Liouville space at scale $\Lambda$, and it can be shown that $L_{S}^{(1)}$ and $Z^{(1)}$ are hermitian operators. Similiar equations can be set up for the current kernel $\Sigma_{\gamma}^{(1)}(E, \omega)$ in leading order by replacing the first vertex on the r.h.s. of Eqs. (130) and (131) by the current vertex.

When integrating the RG equations (130) and (131) up to $\Lambda_{c}$, we obtain a linear contribution in the renormalized coupling $J_{c}$ at scale $\Lambda_{c}$. Since the perturbative treatment in $J_{c}$ for the regime $0<\Lambda<\Lambda_{c}$ can only give corrections $\sim J_{c}^{2}$ to the Liouvillian, we know that the final effective Liouvillian up to $O\left(J_{c}\right)$ is given by

$$
\begin{aligned}
& L_{S}^{e f f}(E, \omega)= \\
& \quad=L_{S}^{(0)}+L_{S}^{(1) c}-(E+i \omega) Z^{(1) c}+O\left(J_{c}^{2}\right),
\end{aligned}
$$

with $L_{S}^{(1) c}=\left.L_{S}^{(1)}\right|_{\Lambda=\Lambda_{c}}$ and $Z^{(1) c}=\left.Z^{(1)}\right|_{\Lambda=\Lambda_{c}}$.

Second order. With the vertex and the Liouvillian in leading order, we can now expand the full RG equations systematically around these reference solutions and calculate the higher orders. The Liouvillian and the vertex are written as an expansion in $J$,

$$
\begin{aligned}
& L_{S}(E, \omega)= \\
& \quad=L_{S}^{(0)}+L_{S}^{(1)}(E, \omega)+L_{S}^{(2)}(E, \omega)+\ldots \\
& \bar{G}_{12}\left(E, \omega, \omega_{1}, \omega_{2}\right)= \\
& \quad=\bar{G}_{12}^{(1)}+\bar{G}_{12}^{(2)}\left(E, \omega, \omega_{1}, \omega_{2}\right)+\ldots
\end{aligned}
$$

where $L_{S}^{(n)}, \bar{G}_{12}^{(n)} \sim J^{n}$ (possibly with additional factors $\sim \ln ^{k} J$ with $k<n$, see below). 
To get an RG equation for $L_{S}^{(2)}(E, \omega)$ and $\bar{G}_{12}^{(2)}\left(E, \omega, \omega_{1}, \omega_{2}\right)$, we insert the expansions 133) and (134) into the r.h.s. of the full RG equations (92) and (93). For the RG of the Liouvillian (vertex), the resolvents are expanded such that we collect all terms in $O\left[\left(\frac{\Delta}{\Lambda}\right)^{k} J^{2}\right]\left\{O\left[\frac{1}{\Lambda}\left(\frac{\Delta}{\Lambda}\right)^{k} J^{2}\right]\right\}$, with $k>1$, and $O\left(\frac{\Delta}{\Lambda} J^{3}\right)$ $\left[O\left(\frac{1}{\Lambda} J^{3}\right)\right]$ on the r.h.s. of the RG equation, where $\Delta$ is some cutoff scale arising from $E_{12}, L_{S}(E)$, or the frequencies. To achieve this, we leave out $L_{S}^{(2)}(E, \omega)$ in the resolvents [leading to terms of $O\left(J^{4}\right)$ on the r.h.s. of the RG equation], and use Eq. (129). This gives for the resolvent

$$
\begin{array}{r}
i \Pi(E, \omega) \approx \frac{1}{\omega-i E+i L_{S}^{(0)}+i L_{S}^{(1)}+(\omega-i E) Z^{(1)}} \\
\quad \approx \frac{1}{1+\frac{Z^{(1)}}{2}} \cdot \frac{1}{\omega-i E+i L_{S}^{(0)}+i \tilde{L}_{S}^{(1)}} \cdot \frac{1}{1+\frac{Z^{(1)}}{2}},
\end{array}
$$

with the hermitian operator

$$
\begin{aligned}
& \tilde{L}_{S}^{(1)}=\frac{1}{1+\frac{Z^{(1)}}{2}} L_{S}^{(1)} \frac{1}{1+\frac{Z^{(1)}}{2}} \\
& \approx L_{S}^{(1)}-\frac{1}{2}\left(Z^{(1)} L_{S}^{(0)}+L_{S}^{(0)} Z^{(1)}\right) .
\end{aligned}
$$

Vertex in second order. We start with the RG equation (93) for the vertex (or equivalently the current vertex by replacing the first vertex by the current vertex in all equations). For the resolvent in the first term on the r.h.s. of this RG equation, we expand (135) in the following way:

$$
\begin{aligned}
& i \Pi\left(E_{12}, \Lambda+\omega+\omega_{1}\right) \approx \\
& \approx \frac{1-Z^{(1)}}{\Lambda}+\left(\frac{1}{\Lambda+\omega+\omega_{1}-i E_{12}+i L_{S}^{(0)}}-\frac{1}{\Lambda}\right) \\
& =\frac{1-Z^{(1)}}{\Lambda}+\frac{d}{d \Lambda} \ln \frac{\Lambda+\omega+\omega_{1}-i E_{12}+i L_{S}^{(0)}}{\Lambda} .
\end{aligned}
$$

Together with the two vertices, this approximation contains systematically all terms of $O\left(\frac{1}{\Lambda} J^{2}\right), O\left(\frac{1}{\Lambda} J^{3}\right)$, and $O\left[\frac{1}{\Lambda}\left(\frac{\Delta}{\Lambda}\right)^{k} J^{2}\right]$, with $k>1$, which are important to calculate the vertex up to $O\left(J^{2}\right)$. The last three terms on the r.h.s. of Eq. (93) are already $\sim J^{3}$, therefore we replace the resolvents by their lowest order term $\sim \frac{1}{\Lambda}$ :

$$
\begin{aligned}
i \Pi\left(E_{23}, \Lambda+\omega+\omega_{3}\right) & \approx \frac{1}{\Lambda+\omega_{3}}, \\
i \Pi\left(E_{11^{\prime} 23}, \Lambda+\omega+\omega_{1}+\omega_{1}^{\prime}+\omega_{3}\right) & \approx \frac{1}{\Lambda+\omega_{3}}, \\
i \Pi\left(E_{12}, \Lambda+\omega+\omega_{1}\right) & \approx \frac{1}{\Lambda} .
\end{aligned}
$$

Note that $\omega_{3}$ is an integration variable and has to be kept in the resolvent. As a result, we get the following RG equation for the vertex in second order in $J$ :

$$
\begin{gathered}
\frac{d \bar{G}_{11^{\prime}}^{(2)}\left(E, \omega, \omega_{1}, \omega_{1}^{\prime}\right)}{d \Lambda}= \\
=\left\{\bar{G}_{12}^{(1)}\left(-\frac{Z^{(1)}}{\Lambda}+\frac{d}{d \Lambda} \ln \frac{\left.\Lambda+\omega+\omega_{1}-i E_{12}+i L_{S}^{(0)}\right)}{\Lambda}\right) \bar{G}_{\overline{2}_{11^{\prime}}}^{(1)}-\left(1 \leftrightarrow 1^{\prime}\right)\right\} \\
\quad+\frac{1}{\Lambda}\left\{\bar{G}_{12}^{(1)} \bar{G}_{\overline{2} 1^{\prime}}^{(2)}\left(E_{12}, \Lambda+\omega+\omega_{1},-\Lambda, \omega_{1}^{\prime}\right)+\bar{G}_{12}^{(2)}\left(E, \omega, \omega_{1}, \Lambda\right) \bar{G}_{\overline{2} 1^{\prime}}^{(1)}-\left(1 \leftrightarrow 1^{\prime}\right)\right\} \\
-\int_{0}^{\Lambda} d \omega_{3}\left(\frac{1}{\Lambda+\omega_{3}}\right)^{2} \bar{G}_{23}^{(1)} \bar{G}_{11^{\prime}}^{(1)} \bar{G}_{\overline{3} \overline{2}}^{(1)}+\frac{1}{\Lambda} \int_{0}^{\Lambda} d \omega_{3} \frac{1}{\Lambda+\omega_{3}}\left\{\bar{G}_{12}^{(1)} \bar{G}_{1^{\prime} 3}^{(1)} \bar{G}_{\overline{3} \overline{2}}^{(1)}-\bar{G}_{23}^{(1)} \bar{G}_{\overline{3} 1}^{(1)} \bar{G}_{1^{\prime} \overline{2}}^{(1)}-\left(1 \leftrightarrow 1^{\prime}\right)\right\} .
\end{gathered}
$$

The first term [Eq. (138)] on the r.h.s. containing the logarithm induces the frequency dependence of the vertex. If we neglect in this term the derivative of $\bar{G}^{(1)}$ with respect to $\Lambda$ (giving rise to terms of $O\left[\frac{1}{\Lambda}\left(\frac{\Delta}{\Lambda}\right)^{k} J^{3}\right]$, with $k>1$, contributing to $\bar{G}^{(3)}$ ), we can decompose the vertex in the following way to solve the above RG equation in $O\left(J^{2}\right)$ :

$$
\bar{G}_{11^{\prime}}^{(2)}\left(E, \omega, \omega_{1}, \omega_{1}^{\prime}\right)=\bar{G}_{11^{\prime}}^{(2 a)}+\bar{G}_{11^{\prime}}^{(2 b)}\left(E, \omega, \omega_{1}, \omega_{1}^{\prime}\right),
$$

with

$$
\begin{aligned}
\bar{G}_{11^{\prime}}^{(2 b)}\left(E, \omega, \omega_{1}, \omega_{1}^{\prime}\right)= \\
=\bar{G}_{12}^{(1)} \ln \frac{\Lambda+\omega+\omega_{1}-i E_{12}+i L_{S}^{(0)}}{\Lambda} \bar{G}_{\overline{2} 1^{\prime}}^{(1)} \\
-\bar{G}_{1^{\prime} 2}^{(1)} \ln \frac{\Lambda+\omega+\omega_{1}^{\prime}-i E_{1^{\prime} 2}+i L_{S}^{(0)}}{\Lambda} \bar{G}_{\overline{2} 1}^{(1)} .
\end{aligned}
$$

Using this solution in the second term [Eq. (139)] on the r.h.s., and neglecting again terms of $O\left[\frac{1}{\Lambda}\left(\frac{\Delta}{\Lambda}\right)^{k} J^{3}\right]$, we can 
use the approximations

$$
\begin{aligned}
\bar{G}_{\overline{2} 1^{\prime}}^{(2 b)}\left(E_{12}, \Lambda+\omega+\omega_{1},-\Lambda, \omega_{1}^{\prime}\right) & \approx-\ln 2 \bar{G}_{1^{\prime} 3}^{(1)} \bar{G}_{\overline{3} \overline{2}}^{(1)} \\
\bar{G}_{12}^{(2 b)}\left(E, \omega, \omega_{1}, \Lambda\right) & \approx-\ln 2 \bar{G}_{23}^{(1)} \bar{G}_{\overline{3} 1}^{(1)}
\end{aligned}
$$

in Eq. (139), which lead to two terms cancelling precisely the second term of Eq. (140). Thus, we obtain the following RG equation for the frequency-independent part $\bar{G}_{11^{\prime}}^{(2 a)}$ of the vertex in $O\left(J^{2}\right)$ :

$$
\begin{aligned}
\frac{d \bar{G}_{11^{\prime}}^{(2 a)}}{d \Lambda}= & \frac{1}{\Lambda}\left\{\bar{G}_{12}^{(1)} \bar{G}_{\overline{2} 1^{\prime}}^{(2 a)}+\bar{G}_{12}^{(2 a)} \bar{G}_{\overline{2} 1^{\prime}}^{(1)}-\left(1 \leftrightarrow 1^{\prime}\right)\right\} \\
& -\frac{1}{\Lambda}\left\{\bar{G}_{12}^{(1)} Z^{(1)} \bar{G}_{\overline{2} 1^{\prime}}^{(1)}-\left(1 \leftrightarrow 1^{\prime}\right)\right\}-\frac{1}{2 \Lambda} \bar{G}_{23}^{(1)} \bar{G}_{11^{\prime}}^{(1)} \bar{G}_{\overline{3} \overline{2}}^{(1)}
\end{aligned}
$$

where the initial condition is given by the second term of the inital condition (75) for the vertex. It can be shown ${ }^{17}$ that the form of the initial condition is preserved [with the vertices given by the leading-order solutions (117) and (118)] if one considers only the first term on the r.h.s. of Eq. (143). Therefore, we decompose the frequencyindependent part of the vertex in second order as

$$
\bar{G}_{11^{\prime}}^{(2 a)}=i \bar{G}_{11^{\prime}}^{\left(2 a_{1}\right)}+\bar{G}_{11^{\prime}}^{\left(2 a_{2}\right)}
$$

with

$$
\bar{G}_{11^{\prime}}^{\left(2 a_{1}\right)}=-\frac{\pi}{2}\left(\bar{G}_{12}^{(1)} \tilde{G}_{\overline{2} 1^{\prime}}^{(1)}-\bar{G}_{1^{\prime} 2}^{(1)} \tilde{G}_{\overline{2} 1}^{(1)}\right),
$$

and $\bar{G}_{11^{\prime}}^{\left(2 a_{2}\right)}$ fulfils the same RG equation (143) as $\bar{G}_{11^{\prime}}^{(2 a)}$, but with zero initial condition. Since no explicit imaginary factors occur in Eq. (143), the decomposition (144) can also be viewed as a decomposition of the vertex into real and complex parts (provided there are no complex terms in the initial condition for the original quantities). Therefore, the two parts have a completely different physical meaning. Whereas the part $\bar{G}_{11^{\prime}}^{\left(2 a_{1}\right)}$ is even important to calculate the rates in second order in $J$ (see below), the part $\bar{G}_{11^{\prime}}^{\left(2 a_{2}\right)}$ denotes a renormalization of the coupling constants in two-loop order, which can lead to logarithmic corrections of the form $\sim J^{2} \ln \frac{J}{J_{0}}$, where $J_{0}$ denotes the original coupling constant (see Sec. IIIB 1. where such terms are explicitly calculated for the Kondo model). Such terms are not well defined in the scaling limit $J_{0} \rightarrow 0$ and, therefore, should be taken together with the leading order vertex by redefining certain characteristic low-energy scales (like the Kondo temperature for the Kondo model). Thus, in the following we will redefine the leading-order vertex by the replacement

$$
\bar{G}_{11^{\prime}}^{(1)} \rightarrow \bar{G}_{11^{\prime}}^{(1)}+\bar{G}_{11^{\prime}}^{\left(2 a_{2}\right)}
$$

and will consider only the part $\bar{G}_{11^{\prime}}^{\left(2 a_{1}\right)}$ explicitly in all equations. We note that it can not generically be shown that the replacement (146) can be accounted for by just renormalizing the Kondo temperature in the lowest-order vertices. For the Kondo model, this can be shown in all orders 31 because only one coupling constant remains in the scaling limit $J_{0} \rightarrow 0, D \rightarrow \infty$, such that $T_{K}$ stays constant. However, for more complicated models including orbital degrees of freedoms, interference effects, etc., it can happen that the matrix structure of $\bar{G}_{11^{\prime}}^{\left(2 a_{2}\right)}$ in Liouville space is different from that of $\bar{G}_{11^{\prime}}^{(1)}$. In this case, new terms can finally arise from the second-order part of the vertices, and their influence might be quite nontrivial.

We note that all equations also hold for the current vertex $\bar{I}^{\gamma(2)}$ by replacing the first vertex in all terms on the r.h.s. of the RG equations by the current vertex. Thereby Eq. (145) is only valid if the trace $\operatorname{Tr}_{S}$ over the local quantum system is taken from the left (which we always implicitly assume for the current vertex and the current kernel).

Liouvillian in second order. We proceed with RG equation (92) for the Liouvillian (or equivalently the current kernel by replacing the first vertex by the current vertex in all equations). Inserting the expansion (134) into this RG equation, using Eqs. (141), (144), and (146), and neglecting all terms of $O\left(J^{4}\right)$ on the r.h.s., we obtain

$$
\begin{aligned}
& \frac{d L_{S}(E, \omega)}{d \Lambda} \approx \bar{G}_{12}^{(1)} \Pi\left(E_{12}, \Lambda+\omega+\omega_{2}\right) \bar{G}_{\overline{2} \overline{1}}^{(1)} \\
& \quad+i \bar{G}_{12}^{(1)} \Pi\left(E_{12}, \Lambda+\omega+\omega_{2}\right) \bar{G}_{\overline{2} \overline{1}}^{\left(2 a_{1}\right)}+i \bar{G}_{12}^{\left(2 a_{1}\right)} \Pi\left(E_{12}, \Lambda+\omega+\omega_{2}\right) \bar{G}_{\overline{2} \overline{1}}^{(1)} \\
& +\bar{G}_{12}^{(1)} \Pi\left(E_{12}, \Lambda+\omega+\omega_{2}\right) \bar{G}_{\overline{2} \overline{1}}^{(2 b)}\left(E_{12}, \Lambda+\omega+\omega_{2},-\omega_{2},-\Lambda\right)+\bar{G}_{12}^{(2 b)}\left(E, \omega, \Lambda, \omega_{2}\right) \Pi\left(E_{12}, \Lambda+\omega+\omega_{2}\right) \bar{G}_{\overline{2} \overline{1}}^{(1)} \\
& \quad-i \bar{G}_{12}^{(1)} \Pi\left(E_{12}, \Lambda+\omega+\omega_{2}\right) \bar{G}_{\overline{2} 3}^{(1)} \Pi\left(E_{13}, \Lambda+\omega+\omega_{3}\right) \bar{G}_{\overline{3} \overline{1}}^{(1)} .
\end{aligned}
$$


For the terms (147), (148), and (150) we need the resolvent integrated over frequency, which, by using
Eqs. (135), (136), (122) and (123), can be expanded as

$$
\begin{aligned}
& i \int_{0}^{\Lambda} d \omega_{2} \Pi\left(E_{12}, \Lambda+\omega+\omega_{2}\right) \approx\left(1-\frac{Z^{(1)}}{2}\right) \mathcal{K}_{\Lambda}\left(E_{12}+i \omega-L_{S}^{(0)}-\tilde{L}_{S}^{(1)}\right)\left(1-\frac{Z^{(1)}}{2}\right) \\
& \approx \frac{i}{2 \Lambda}\left(1-\frac{Z^{(1)}}{2}\right)\left(E_{12}+i \omega-L_{S}^{(0)}-\tilde{L}_{S}^{(1)}\right)\left(1-\frac{Z^{(1)}}{2}\right)+\tilde{\mathcal{K}}_{\Lambda}\left(E_{12}+i \omega-L_{S}^{(0)}-\tilde{L}_{S}^{(1)}\right) \\
& \approx \frac{i}{2 \Lambda}\left(E_{12}+i \omega-L_{S}^{(0)}\right)-\frac{i}{2 \Lambda}\left(\left(E_{12}+i \omega\right) Z^{(1)}+L_{S}^{(1)}-Z^{(1)} L_{S}^{(0)}-L_{S}^{(0)} Z^{(1)}\right)+\tilde{\mathcal{K}}_{\Lambda}\left(E_{12}+i \omega-L_{S}^{(0)}\right) .
\end{aligned}
$$

The first term is of order $O\left(\frac{\Delta}{\Lambda}\right)$, the second one of order $O\left(\frac{\Delta}{\Lambda} J\right)$, and the last one contains terms of $O(1)$ and $O\left[\left(\frac{\Delta}{\Lambda}\right)^{k}\right]$, with $k>1$. Therefore, when multiplied with $J^{n}$ and integrated, the first term gives a contribution of order $O\left(\Delta J^{n-1}\right)$, the second one leads to $O\left(\Delta J^{n}\right)$, and the last one gives $O\left(\Lambda_{0} J^{n}\right)$ or $O\left(\Delta J^{n}\right)$. Thereby, the terms $\sim \Lambda_{0} J^{n}$ are cancelled by the initial condition from the first RG step (see below). Therefore, for the calculation of $\frac{d}{d \Lambda} L_{S}^{(2)}(E, \omega)$, the terms (147) and (148) give rise to

$$
\begin{aligned}
\frac{d L_{S}^{(2)}(E, \omega)}{d \Lambda} \rightarrow & -i \frac{d}{d \Lambda}\left\{\bar{G}_{12}^{(1)} \tilde{F}_{\Lambda}\left(E_{12}+i \omega-L_{S}^{(0)}\right) \bar{G}_{\overline{2} \overline{1}}^{(1)}\right\} \\
& +\frac{i}{2 \Lambda}\left\{\bar{G}_{12}^{(1)}\left(E_{12}+i \omega-L_{S}^{(0)}\right) \bar{G}_{\overline{2} \overline{1}}^{\left(2 a_{1}\right)}+\bar{G}_{12}^{\left(2 a_{1}\right)}\left(E_{12}+i \omega-L_{S}^{(0)}\right) \bar{G}_{\overline{2} \overline{1}}^{(1)}\right\} \\
& -\frac{1}{2 \Lambda} \bar{G}_{12}^{(1)}\left\{\left(E_{12}+i \omega\right) Z^{(1)}+L_{S}^{(1)}-Z^{(1)} L_{S}^{(0)}-L_{S}^{(0)} Z^{(1)}\right\} \bar{G}_{\overline{2} \overline{1}}^{(1)} .
\end{aligned}
$$

As is shown in Appendix A the other two terms, Eqs. (149) and (150), have nearly no effect when expanded systematically, one just has to replace the function $\tilde{F}_{\Lambda}(z)$ in Eq. (152) by the function

$$
\tilde{F}_{\Lambda}^{\prime}(z)=\tilde{F}_{\Lambda}(z)-\frac{i z}{2} \ln 2
$$

According to the three terms Eqs. (152)-(154), we decompose

$$
\begin{aligned}
L_{S}^{(2)}(E, \omega)= & L_{S}^{(2 a)}(E, \omega)+L_{S}^{(2 b)}+L_{S}^{(2 c)} \\
& -(E+i \omega)\left(Z^{(2 b)}+Z^{(2 c)}\right)
\end{aligned}
$$

with

$$
\begin{aligned}
L_{S}^{(2 a)}(E, \omega)= & -i \bar{G}_{12}^{(1)} \tilde{F}_{\Lambda}^{\prime}\left(E_{12}+i \omega-L_{S}^{(0)}\right) \bar{G}_{\overline{2} \overline{1}}^{(1)}, \\
\frac{d L_{S}^{(2 b)}}{d \Lambda}= & \frac{i}{2 \Lambda}\left\{\bar{G}_{12}^{(1)}\left(\bar{\mu}_{12}-L_{S}^{(0)}\right) \bar{G}_{\overline{2} \overline{1}}^{\left(2 a_{1}\right)}+\right. \\
& \left.+\bar{G}_{12}^{\left(2 a_{1}\right)}\left(\bar{\mu}_{12}-L_{S}^{(0)}\right) \bar{G}_{\overline{2} \overline{1}}^{(1)}\right\}, \\
\frac{d Z^{(2 b)}}{d \Lambda}= & -\frac{i}{2 \Lambda}\left\{\bar{G}_{12}^{(1)} \bar{G}_{\overline{2} \overline{1}}^{\left(2 a_{1}\right)}+\bar{G}_{12}^{\left(2 a_{1}\right)} \bar{G}_{\overline{2} \overline{1}}^{(1)}\right\}, \\
\frac{d L_{S}^{(2 c)}}{d \Lambda}= & -\frac{1}{2 \Lambda} \bar{G}_{12}^{(1)}\left\{\left(\bar{\mu}_{12} Z^{(1)}+L_{S}^{(1)}-\right.\right. \\
& \left.-Z^{(1)} L_{S}^{(0)}-L_{S}^{(0)} Z^{(1)}\right\} \bar{G}_{\overline{2} \overline{1}}^{(1)}, \\
\frac{d Z_{S}^{(2 c)}}{d \Lambda}= & \frac{1}{2 \Lambda} \bar{G}_{12}^{(1)} Z^{(1)} \bar{G}_{\overline{2} \overline{1}}^{(1)} .
\end{aligned}
$$

Note that, according to Eq. (126), the initial value of $L_{S}^{(2 a)}(E, \omega)$ is given by

$$
\left.L_{S}^{(2 a)}(E, \omega)\right|_{\Lambda=\Lambda_{0}}=-\left.i \ln (2) \Lambda_{0} \bar{G}_{12}^{(1)} \bar{G}_{\overline{2} \overline{1}}^{(1)}\right|_{\Lambda=\Lambda_{0}},
$$

which coincides with the first term of the inital condition 
(74) from the first RG step if we choose

$$
\Lambda_{0}=\frac{\pi^{2}}{16 \ln (2)} D \text {. }
$$

One can group the various terms occurring in Eqs. (156)-1161) regarding the occurence of the complex factor $i$. The RG equations (160) and (161) do not contain an explicit factor $i$ and integrating them up to $\Lambda_{c}$, one generates a second-order contribution which is negligible compared to the first-order terms (130) and (131) (up to this value of the cutoff parameter, no logarithmic contributions are generated, i.e., the terms just have one factor more in $J$ ). In contrast, the second-order terms containing the factor $i$ are not negligible because they do not occur in first order. They are generated by Eq. (157) [with $F_{\Lambda}^{\prime}(z)$ replaced by its real value] and by Eqs. (158) and (159). This is a general rule that the terms containing the factor $i$ are always generated one order higher in $J$ compared to those without this factor.

\section{2. $R G$ below $\Lambda_{c}$}

The two-loop RG until $\Lambda_{c}$ has resummed all leading and subleading logarithmic contributions in $\ln \frac{D}{\Lambda_{c}}$ into the renormalized vertices. This means that we have considered all terms of the form

$$
J_{0}^{k} \ln ^{l} \frac{D}{\Lambda_{c}} \quad, \quad l=k-1, k-2,
$$

for the Liouvillian and the vertices, where $J_{0}$ denotes the original coupling constant. The terms with $l=k-1$ and $l=k-2$ are defined in our terminology as one-loop and two-loop terms, respectively, irrespective of the topology of the diagrams, which is not a unique definition and depends on the formalism used. Roughly speaking, at $\Lambda=\Lambda_{c}$, the band width $D$ has been replaced by an effective band width $\Lambda_{c}$ and the bare coupling constant is replaced by a renormalized one $J_{c}$ (including one-loop and two-loop renormalizations). This eliminates all the logarithmic contributions of Eq. (164), and a simple power series in $J_{c}$ remains, which is well defined for $J_{c} \ll 1$, as described in detail at the beginning of Sec. IIC

As a consequence, we solve the RG equations perturbatively in $J_{c}$ in the regime $0<\Lambda<\Lambda_{c}$. Furthermore, we replace the Liouvillian in the resolvents by the full effective Liouvillian $L_{S}^{e f f}(E, \omega)$, and we use the approximation (54)

$$
\Pi(E, \omega) \approx \frac{\tilde{Z}}{E+i \omega-\tilde{L}_{S}},
$$

together with the convention (55). The corrections to this approximation are at least of order $O\left(J_{c}\right)$ and contain no poles in the variable $E+i \omega$ (at least if we assume that the projectors on the eigenvectors do not contain poles or do not contribute). Therefore, when inserted in the
RG equations, these corrections lead to terms of order $O\left(J_{c}^{3}\right)$ without any logarithmic enhancement, which we neglect in the following. The eigenvalues of $\tilde{L}_{S}$ are given by $\tilde{h}_{i}-i \tilde{\Gamma}_{i}$ with $\tilde{\Gamma}_{i}>0$. These eigenvalues are finally calculated self-consistently from Eq. (51). In contrast, the $Z$ factor is expanded as

$$
\tilde{Z}=1+\tilde{Z}^{(1)}+O\left(J_{c}^{2}\right),
$$

where $\tilde{Z}^{(1)} \sim J_{c} \ll 1$. This first-order correction to the $Z$ factor can in principle be calculated from the effective Liouvillian up to first order in $J_{c}$, given by the result (132). If the eigenvalues of $L_{S}^{(0)}$ are separated well compared to the first-order corrections to the effective Liouvillian, we get

$$
\tilde{Z}^{(1)}=-Z_{d}^{(1) c},
$$

where $Z_{d}^{(1) c}$ is the diagonal part of $Z^{(1) c}$ with respect to the eigenbasis of $L_{S}^{(0)}$. However, we will see later that $\tilde{Z}^{(1)}$ does not enter our final result so its precise value is of no relevance.

In the RG equations, the variable $E$ is replaced by $E_{1 \ldots n}$ and $\omega$ is the imaginary part of the Laplace variable plus the integration variables (the cutoff $\Lambda$ and some Masubara frequencies). Thus, in the end the low-energy cutoff will be given by an expression of the form

$$
\Delta_{1 \ldots n}=E_{1 \ldots n}+i \omega-\tilde{L}_{S},
$$

where from now on $\omega$ is the imaginary part of the original Laplace variable. Resonance positions are defined by $E_{1 \ldots n}=\tilde{h}_{i}$, see Eq. (114). At these points, logarithmic terms of the form

$$
J_{c}^{k} \ln ^{l} \frac{\Lambda_{c}}{|\Delta|} \quad, \quad l=k-1, k-2, \ldots, 0,
$$

are generated with $\Delta \equiv E_{1 \ldots n}-\tilde{h}_{i}+i \tilde{\Gamma}_{i}$. At resonance, the logarithmic terms are of order $J_{c}^{k} \ln ^{l} J_{c}$ since $\tilde{\Gamma}_{i} \sim \Lambda_{c} J_{c}^{2}$, leading to enhanced, but still perturbative corrections.

Our first aim is to collect all terms of the form (169) with $k \leq 3$ and $l=k-1, k-2$, i.e., all terms of the form

$$
\begin{aligned}
& O(1), O\left(J_{c}\right), O\left(J_{c}^{2}\right), O\left(J_{c}^{2} \ln \frac{\Lambda_{c}}{|\Delta+i \Gamma|}\right), \\
& O\left(J_{c}^{3} \ln \frac{\Lambda_{c}}{|\Delta+i \Gamma|}\right), O\left(J_{c}^{3} \ln ^{2} \frac{\Lambda_{c}}{|\Delta+i \Gamma|}\right) .
\end{aligned}
$$

Only terms of $O\left(J_{c}^{3}\right)$ without a logarithmic factor are neglected [note that approximation (165) for the resolvent is already neglecting such terms]. Therefore, it is not necessary to calculate the Liouvillian in third order in $J$ for $\Lambda>\Lambda_{c}$ since no logarithmic contributions are generated up to $\Lambda_{c}$. Furthermore, for $\Lambda<\Lambda_{c}$, we see that the lowest order term on the r.h.s of the RG equation (92) for the Liouvillian is already of $O\left(J_{c}^{2}\right)$. Therefore, we need 
the vertices only up to $O\left(J_{c}^{2}\right)$, and it is not necessary to calculate the vertices up to third order for $\Lambda>\Lambda_{c}$.

Vertices. We start with the perturbative expansion for the vertices. Up to order $O\left(J_{c}^{2}\right)$, we get from the RG equation (93) together with Eqs. (165), (166), and (168)

$$
\begin{aligned}
& \frac{d \bar{G}_{11^{\prime}}\left(E, \omega, \omega_{1}, \omega_{1}^{\prime}\right)}{d \Lambda}= \\
& \quad=\left\{\bar{G}_{12}^{(1) c} \frac{1}{\Lambda+\omega_{1}-i \Delta_{12}} \bar{G}_{\overline{21^{\prime}}}^{(1) c}-\left(1 \leftrightarrow 1^{\prime}\right)\right\} .
\end{aligned}
$$

Integrating this equation from $\Lambda=\Lambda_{c}$ to $\Lambda$, we obtain in $O\left(J_{c}^{2}\right)$

$$
\begin{aligned}
& \bar{G}_{11^{\prime}}^{(2)}\left(E, \omega, \omega_{1}, \omega_{1}^{\prime}\right)=\bar{G}_{11^{\prime}}^{(2) c}\left(E, \omega, \omega_{1}, \omega_{1}^{\prime}\right)- \\
& \quad-\left\{\bar{G}_{12}^{(1) c} \ln \frac{\Lambda_{c}+\omega_{1}-i \Delta_{12}}{\Lambda+\omega_{1}-i \Delta_{12}} \bar{G}_{\overline{2} 1^{\prime}}^{(1) c}-\left(1 \leftrightarrow 1^{\prime}\right)\right\},
\end{aligned}
$$

where $\bar{G}_{11^{\prime}}^{(2) c}\left(E, \omega, \omega_{1}, \omega_{1}^{\prime}\right)$ is the value of the vertex in second order at $\Lambda=\Lambda_{c}$. Inserting this value from Eqs. (141),
(142), and (144), we find up to $O\left(J_{c}^{2}\right)$

$$
\begin{aligned}
& \bar{G}_{11^{\prime}}^{(2)}\left(E, \omega, \omega_{1}, \omega_{1}^{\prime}\right)=i \bar{G}_{11^{\prime}}^{\left(2 a_{1}\right) c}+ \\
& +\left\{\bar{G}_{12}^{(1) c} \ln \frac{\Lambda+\omega_{1}-i \Delta_{12}}{\Lambda_{c}} \bar{G}_{\overline{2} 1^{\prime}}^{(1) c}-\left(1 \leftrightarrow 1^{\prime}\right)\right\},
\end{aligned}
$$

where we have used that $\Delta_{12}=E_{12}+i \omega-L_{S}^{(0)}+O\left(J_{c}\right)$. Note that the part $\bar{G}_{11^{\prime}}^{\left(2 a_{2}\right)}$ is always taken together with $\bar{G}_{11^{\prime}}^{(1)}$, see Eq. (146), therefore it does not occur in Eq. (172).

Liouvillian. We proceed with the perturbative expansion for the Liouvillian given by the RG equation (92). In the second term on the r.h.s. of this RG equation, we can replace the vertex by the lowest-order term $\bar{G}^{(1) c}$ and the resolvent by $\Pi(E, \omega) \rightarrow \frac{1}{E+i \omega-\tilde{L}_{S}}$, according to Eq. (165). In the first term on the r.h.s., we have to consider the first-order correction $\tilde{Z}^{(1)}$ of the $Z$ factor as well, and we have to use the second-order term for the vertex given by Eq. (172), which gives

$$
\begin{aligned}
\bar{G}_{12}^{(2)}\left(E, \omega, \Lambda, \omega_{2}\right) & =i \bar{G}_{12}^{\left(2 a_{1}\right) c}+\bar{G}_{13}^{(1) c} \ln \frac{2 \Lambda-i \Delta_{13}}{\Lambda_{c}} \bar{G}_{\overline{3} 2}^{(1) c}-\bar{G}_{23}^{(1) c} \ln \frac{\Lambda+\omega_{2}-i \Delta_{23}}{\Lambda_{c}} \bar{G}_{\overline{\overline{3}} 1}^{(1) c}, \\
\bar{G}_{\overline{2} \overline{1}}^{(2)}\left(E_{12}, \Lambda+\omega+\omega_{2},-\omega_{2},-\Lambda\right) & =i \bar{G}_{\overline{2} \overline{1}}^{\left(2 a_{1}\right) c}+\bar{G}_{\overline{2} 3}^{(1) c} \ln \frac{2 \Lambda-i \Delta_{13}}{\Lambda_{c}} \bar{G}_{\overline{3} \overline{1}}^{(1) c}-\bar{G}_{\overline{1} 3}^{(1) c} \ln \frac{\Lambda+\omega_{2}-i \Delta_{23}}{\Lambda_{c}} \bar{G}_{\overline{3} \overline{2}}^{(1) c} .
\end{aligned}
$$

For the frequency integral over the resolvent, we use

$$
\int_{0}^{\Lambda} d \omega_{2} \frac{1}{i \Lambda+i \omega_{2}+\Delta}=-i \mathcal{K}_{\Lambda}(\Delta)=-i \ln \frac{2 \Lambda-i \Delta}{\Lambda-i \Delta}
$$

Using these replacements in Eq. (92) and collecting the various terms according to their order in $J_{c}$, we obtain up to $O\left(J_{c}^{3}\right)$

$$
L_{S}(E, \omega)=L_{S}^{(0)}+L_{S}^{(1) c}(E, \omega)+L_{S}^{(2)}(E, \omega)+L_{S}^{(3 a)}(E, \omega)+L_{S}^{(3 b)}(E, \omega),
$$

with

$$
\begin{aligned}
\frac{d L_{S}^{(2)}(E, \omega)}{d \Lambda}= & -i \bar{G}_{12}^{(1) c} \mathcal{K}_{\Lambda}\left(\Delta_{12}\right) \bar{G}_{\overline{2} \overline{1}}^{(1) c} \\
\frac{d L_{S}^{(3 a)}(E, \omega)}{d \Lambda}= & -\frac{i}{2} \bar{G}_{12}^{(1) c}\left\{\tilde{Z}^{(1)} \mathcal{K}_{\Lambda}\left(\Delta_{12}\right)+\mathcal{K}_{\Lambda}\left(\Delta_{12}\right) \tilde{Z}^{(1)}\right\} \bar{G}_{\overline{2} \overline{1}}^{(1) c} \\
& +\bar{G}_{12}^{(1) c} \mathcal{K}_{\Lambda}\left(\Delta_{12}\right) \bar{G}_{\overline{2} \overline{1}}^{\left(2 a_{1}\right) c}+\bar{G}_{12}^{\left(2 a_{1}\right) c} \mathcal{K}_{\Lambda}\left(\Delta_{12}\right) \bar{G}_{\overline{2} \overline{1}}^{(1) c} \\
\frac{d L_{S}^{(3 b)}(E, \omega)}{d \Lambda}= & -i \bar{G}_{12}^{(1) c} \mathcal{K}_{\Lambda}\left(\Delta_{12}\right) \bar{G}_{\overline{2} 3}^{(1) c} \ln \frac{2 \Lambda-i \Delta_{13}}{\Lambda_{c}} \bar{G}_{\overline{3} \overline{1}}^{(1) c}-i \bar{G}_{12}^{(1) c} \ln \frac{2 \Lambda-i \Delta_{12}}{\Lambda_{c}} \bar{G}_{\overline{2} 3}^{(1) c} \mathcal{K}_{\Lambda}\left(\Delta_{13}\right) \bar{G}_{\overline{3} \overline{1}}^{(1) c} \\
-i \bar{G}_{12}^{(1) c} \int_{\Lambda}^{2 \Lambda} & d \omega_{2}\left\{\frac{1}{\omega_{2}-i \Delta_{12}} \bar{G}_{\overline{2} 3}^{(1) c} \ln \frac{\omega_{2}-i \Delta_{13}}{\Lambda_{c}}+\ln \frac{\omega_{2}-i \Delta_{12}}{\Lambda_{c}} \bar{G}_{\overline{2} 3}^{(1) c} \frac{1}{\omega_{2}-i \Delta_{13}}\right\} \bar{G}_{\overline{3} \overline{1}}^{(1) c} \\
& +i \bar{G}_{12}^{(1) c} \mathcal{K}_{\Lambda}\left(\Delta_{12}\right) \bar{G}_{\overline{2} 3}^{(1) c} \mathcal{K}_{\Lambda}\left(\Delta_{13}\right) \bar{G}_{\overline{3} \overline{1}}^{(1) c} .
\end{aligned}
$$

Using

$$
\int_{\Lambda}^{2 \Lambda} d x\left\{\frac{1}{x+a}\left(\ln \frac{x+b}{\Lambda_{c}}\right)+\left(\ln \frac{x+a}{\Lambda_{c}}\right) \frac{1}{x+b}\right\}=\ln \frac{2 \Lambda+a}{\Lambda_{c}} \ln \frac{2 \Lambda+b}{\Lambda_{c}}-\ln \frac{\Lambda+a}{\Lambda_{c}} \ln \frac{\Lambda+b}{\Lambda_{c}}
$$


for the third term on the r.h.s. of Eq. (179), we obtain after some manipulations

$$
\frac{d L_{S}^{(3 b)}(E, \omega)}{d \Lambda}=-2 i \bar{G}_{12}^{(1) c}\left\{\ln \frac{2 \Lambda-i \Delta_{12}}{\Lambda_{c}} \bar{G}_{\overline{2} 3}^{(1) c} \ln \frac{2 \Lambda-i \Delta_{13}}{\Lambda_{c}}-\ln \frac{\Lambda-i \Delta_{12}}{\Lambda_{c}} \bar{G}_{\overline{2} 3}^{(1) c} \ln \frac{\Lambda-i \Delta_{13}}{\Lambda_{c}}\right\} \bar{G}_{\overline{3} \overline{1}}^{(1) c} .
$$

Equations (177) and (178) can be integrated easily from $\Lambda=\Lambda_{c}$ to $\Lambda=0$ by using

$$
\mathcal{K}_{\Lambda}(\Delta)=\frac{d}{d \Lambda} F_{\Lambda}(\Delta) \quad, \quad F_{\Lambda}(\Delta)=\tilde{F}_{\Lambda}^{\prime}(\Delta)+\frac{i \Delta}{2}\left(\ln \frac{\Lambda}{-2 i \Delta}+1\right)
$$

where $\tilde{F}_{\Lambda}^{\prime}(\Delta)$ is defined by Eqs. (155) and (125). Using $F_{\Lambda=0}(\Delta)=-\frac{i \Delta}{2} \ln 2$, we get

$$
\int_{\Lambda_{c}}^{0} d \Lambda \mathcal{K}_{\Lambda}(\Delta)=-\tilde{F}_{\Lambda_{c}}^{\prime}(\Delta)-\frac{i \Delta}{2}\left(\ln \frac{\Lambda_{c}}{-i \Delta}+1\right)
$$

The value of the Liouvillian at $\Lambda=\Lambda_{c}$ in second order has to be taken from Eqs. (156)-(159). When integrating Eq. (177), the part from the first term on the r.h.s. of Eq. (183) cancels term (157) for $L_{S}^{(2 a) c}(E, \omega)$ if we neglect the difference between $L_{S}^{(0)}$ and $\tilde{L}_{S}$ [leading to terms of order $O\left(J_{c}^{3}\right)$ ]. Thus, for $\Lambda=0$, we obtain the contributions

$$
\begin{aligned}
L_{S}^{(1)}(E, \omega)_{\Lambda=0}= & L_{S}^{(1) c}-(E+i \omega) Z^{(1) c} \\
L_{S}^{(2)}(E, \omega)_{\Lambda=0}= & L_{S}^{(2 b) c}+L_{S}^{(2 c) c}-(E+i \omega)\left(Z^{(2 b) c}+Z^{(2 c) c}\right)-\frac{1}{2} \bar{G}_{12}^{(1) c} \Delta_{12}\left(\ln \frac{\Lambda_{c}}{-i \Delta_{12}}+1\right) \bar{G}_{\overline{2} \overline{1}}^{(1) c} \\
L_{S}^{(3 a)}(E, \omega)_{\Lambda=0}= & -\frac{1}{4} \bar{G}_{12}^{(1) c}\left\{\tilde{Z}^{(1)} \Delta_{12}\left(\ln \left|\frac{\Lambda_{c}}{\Delta_{12}}\right|\right)+\Delta_{12}\left(\ln \left|\frac{\Lambda_{c}}{\Delta_{12}}\right|\right) \tilde{Z}^{(1)}\right\} \bar{G}_{\overline{2} \overline{1}}^{(1) c} \\
& -i \frac{1}{2} \bar{G}_{12}^{(1) c} \Delta_{12}\left(\ln \left|\frac{\Lambda_{c}}{\Delta_{12}}\right|\right) \bar{G}_{\overline{2} \overline{1}}^{\left(2 a_{1}\right) c}-i \frac{1}{2} \bar{G}_{12}^{\left(2 a_{1}\right) c} \Delta_{12}\left(\ln \left|\frac{\Lambda_{c}}{\Delta_{12}}\right|\right) \bar{G}_{\overline{2} \overline{1}}^{(1) c}
\end{aligned}
$$

Thereby, we have only included the logarithmic terms in $L_{S}^{(3 a)}$, i.e., terms $\sim J_{c}^{3}$ without a logarithmic factor are neglected.

Finally, integrating Eq. (181), we obtain

$$
L_{S}^{(3 b)}(E, \omega)_{\Lambda=0}=-i \Lambda_{c} \int_{0}^{1} d x \bar{G}_{12}^{(1) c} \ln \left(x-i \frac{\Delta_{12}}{\Lambda_{c}}\right) \bar{G}_{\overline{2} 3}^{(1) c} \ln \left(x-i \frac{\Delta_{13}}{\Lambda_{c}}\right) \bar{G}_{\overline{3} \overline{1}}^{(1) c}
$$

Neglecting again terms $\sim J_{c}^{3}$, the double logarithmic integral can be replaced by (with $a \equiv-i \frac{\Delta_{12}}{\Lambda_{c}}$ and $b \equiv-i \frac{\Delta_{13}}{\Lambda_{c}}$ )

$$
\int_{0}^{1} d x \ln (x+a) \ln (x+b) \approx-\frac{1}{2} a \ln ^{2} a-\frac{1}{2} b \ln ^{2} b+a \ln a+b \ln b+\frac{1}{2} a \ln ^{2} \frac{a}{b}-a \ln \frac{a}{b} \quad, \quad \text { for } \quad|a|<|b| \quad,
$$

and analog for $|a|>|b|$ by interchanging $a \leftrightarrow b$. For some special cases, (188) can be written as

$$
\int_{0}^{1} d x \ln (x+a) \ln (x+b) \approx\left\{\begin{array}{cl}
-\frac{1}{2} a \ln ^{2} a-\frac{1}{2} b \ln ^{2} b+a \ln a+b \ln b & \text { for } \quad|a| \sim|b| \ll 1 \\
-a \ln a \ln b & \text { for } \quad|a| \ll|b| \sim 1
\end{array} .\right.
$$

Equations (184)-(187) together with Eqs. (188)-(189) are the final results for the effective Liouvillian (or the current kernel if the first vertex in all terms is replaced by the current vertex) for a generic model of a quantum dot in the Coulomb blockade regime. The vertices $\bar{G}_{11^{\prime}}^{(1) c}$ and $\bar{G}_{11^{\prime}}^{\left(2 a_{1}\right) c}$ at $\Lambda=\Lambda_{c}$ follow from Eqs. (115) and (145). The firstorder quantities $L_{S}^{(1) c}$ and $Z^{(1) c}$ follow from the solution of the RG equations (130) and (131) at $\Lambda=\Lambda_{c}$, and the second-order terms $L_{S}^{(2 b) c}, L_{S}^{(2 c) c}, Z^{(2 b) c}$, and $Z^{(2 c) c}$ are determined by the RG equations (158)-(161).

Looking back at Eqs. (177)-(179), we see that the third-order terms involving three vertices on the r.h.s. of the RG equations enter only explicitly via the last term on the r.h.s. of Eq. (179). This part is of $O\left(J_{c}^{3}\right)$ and does not contain any logarithmic contribution since $K_{\Lambda}\left(\Delta_{12}\right) \rightarrow i \frac{\Lambda}{\Delta_{12}}$ for $\Lambda \rightarrow 0$. This means that the logarithmic contributions are only generated by the terms in second order in the renormalized vertices, but including their corrections in second order in $J_{c}$ via Eq. (172). Both the imaginary parts $i \bar{G}^{\left(2 a_{1}\right) c}$ and the frequency dependence generate logarithmic contributions. Implicitly, third-order terms in the vertices are also present in the last term on the r.h.s. of the two-loop RG equation (143), which determines the contribution $\bar{G}^{\left(2 a_{2}\right)}$, see Eq. (146). As already mentioned after Eq. (146), it may happen 
for more complicated models than the Kondo model that this part can change the leading-order vertex in a nontrivial way (i.e., not only by a change of the Kondo temperature) leading to new logarithmic contributions not expected from the matrix structure of the leading order vertices.

For $\omega=0$ and away from resonance $\left|\Delta_{12}\right|,\left|\Delta_{13}\right| \gg \tilde{\Gamma}$, we have $a=-i \bar{a}$ and $b=-i \bar{b}$, with $\bar{a}$ and $\bar{b}$ being real. In this case, we get for Eqs. (188) and (189)

$$
\begin{aligned}
& -i \int_{0}^{1} d x \ln (x-i \bar{a}) \ln (x-i \bar{b}) \approx \frac{1}{2} \bar{a} \ln ^{2}|\bar{a}|+\frac{1}{2} \bar{b} \ln ^{2}|\bar{b}|-\bar{a} \ln |\bar{a}|-\bar{b} \ln |\bar{b}|-\frac{1}{2} \bar{a} \ln ^{2}\left|\frac{\bar{a}}{\bar{b}}\right| \\
& -i \frac{\pi}{2}\left(|\bar{a}| \ln |\bar{a}|+|\bar{b}| \ln |\bar{b}|-|\bar{a}|(1-\operatorname{sign} \bar{a} \operatorname{sign} \bar{b}) \ln \left|\frac{\bar{a}}{\bar{b}}\right|\right) \text {, for }|\bar{a}|<|\bar{b}| \\
& \approx\left\{\begin{array}{cll}
\frac{1}{2} \bar{a} \ln ^{2}|\bar{a}|+\frac{1}{2} \bar{b} \ln ^{2}|\bar{b}|-\bar{a} \ln |\bar{a}|-\bar{b} \ln |\bar{b}|-i \frac{\pi}{2}(|\bar{a}| \ln |\bar{a}|+|\bar{b}| \ln |\bar{b}|) & \text { for }|\bar{a}| \sim|\bar{b}| \ll 1 \\
-i \frac{\pi}{2} \bar{a} \operatorname{sign} \bar{b} \ln |\bar{a}| & \text { for } \quad|\bar{a}| \ll|\bar{b}| \sim 1
\end{array} .\right.
\end{aligned}
$$

For $\omega=0$, we see that the terms containing explicitly the complex factor $i$ start always one power less in the logarithm compared to those without the factor $i$. The terms without the factor $i$ start with the logarithmic contribution $\sim J_{c}^{2} \ln \frac{\Lambda_{c}}{|\Delta+i \tilde{\Gamma}|}$, whereas the ones with the factor $i$ start with terms $\sim i J_{c}^{3} \ln \frac{\Lambda_{c}}{|\Delta+i \tilde{\Gamma}|}$. Therefore, in the following section, where we apply the general formalism to the Kondo model, we will restrict ourselves only to those terms. This means that for $\omega=0$ we take into account all terms of order $O(1), O\left(J_{c}\right)$ and $O\left(J_{c}^{2} \ln \frac{\Lambda_{c}}{|\Delta+i \Gamma|}\right)$ without the factor $i$, and all terms of order $O\left(J_{c}^{2}\right)$ and $O\left(J_{c}^{3} \ln \frac{\Lambda_{c}}{|\Delta+i \tilde{\Gamma}|}\right)$ with the factor $i$. Thus, we can neglect the contributions from $L_{S}^{(2 c) c}$ and $Z^{(2 c) c}$ in Eq. (185) [according to Eqs. (160) and (161)], and the first two terms on the r.h.s. of Eq. (186). Therefore, the first-order correction $\tilde{Z}^{(1)}$ is not important for the final result. Furthermore, we can neglect all real terms in Eqs. (190) and (191). This leads to the final equations

$$
\begin{aligned}
L_{S}^{(1)}(E)_{\Lambda=0} & =L_{S}^{(1) c}-E Z^{(1) c} \\
L_{S}^{(2)}(E)_{\Lambda=0} & \approx L_{S}^{(2 b) c}-E Z^{(2 b) c}-i \frac{\pi}{4} \bar{G}_{12}^{(1) c}\left|\Delta_{12}\right| \bar{G}_{\overline{2} \overline{1}}^{(1) c}-\frac{1}{2} \bar{G}_{12}^{(1) c} \Delta_{12}\left(\ln \left|\frac{\Lambda_{c}}{\Delta_{12}}\right|\right) \bar{G}_{\overline{2} \overline{1}}^{(1) c}, \\
L_{S}^{(3 a)}(E)_{\Lambda=0} & \approx-i \frac{1}{2} \bar{G}_{12}^{(1) c} \Delta_{12}\left(\ln \left|\frac{\Lambda_{c}}{\Delta_{12}}\right|\right) \bar{G}_{\overline{2} \overline{1}}^{\left(2 a_{1}\right) c}-i \frac{1}{2} \bar{G}_{12}^{\left(2 a_{1}\right) c} \Delta_{12}\left(\ln \left|\frac{\Lambda_{c}}{\Delta_{12}}\right|\right) \bar{G}_{\overline{2} \overline{1}}^{(1) c}, \\
L_{S}^{(3 b)}(E)_{\Lambda=0} & \approx-i \Lambda_{c} \int_{0}^{1} d x \bar{G}_{12}^{(1) c} \ln \left(x-i \frac{\Delta_{12}}{\Lambda_{c}}\right) \bar{G}_{\overline{2} 3}^{(1) c} \ln \left(x-i \frac{\Delta_{13}}{\Lambda_{c}}\right) \bar{G}_{\overline{3} \overline{1}}^{(1) c},
\end{aligned}
$$

with

$$
\Delta_{i j}=E_{i j}-\tilde{L}_{S}
$$

and

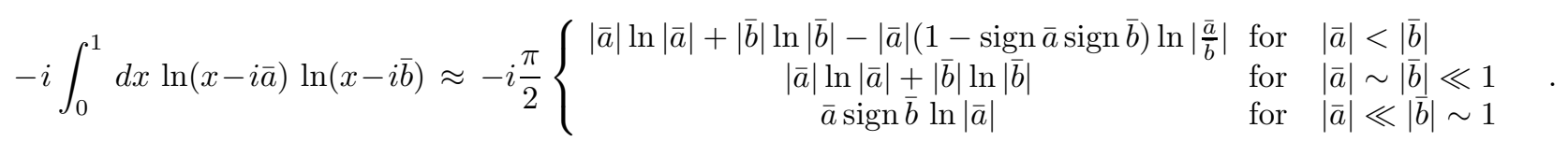

Close to resonance, where the effect of $\tilde{\Gamma}$ can not be neglected, we have to consider the imaginary parts of the eigenvalues of $\tilde{L}_{S}$ as well. If an eigenvalue $\tilde{h}-i \tilde{\Gamma}$ occurs, we have to replace $\Delta_{i j} \rightarrow E_{i j}-\tilde{h}+i \tilde{\Gamma}$, and

$$
\left|\Delta_{i j}\right| \rightarrow\left(E_{i j}-\tilde{h}\right) \frac{2}{\pi} \arctan \frac{E_{i j}-\tilde{h}}{\tilde{\Gamma}} \quad, \quad \operatorname{sign} \Delta_{i j} \rightarrow \frac{2}{\pi} \arctan \frac{E_{i j}-\tilde{h}}{\tilde{\Gamma}} \quad, \quad \ln \left|\Delta_{i j}\right| \rightarrow \ln \left|E_{i j}-\tilde{h}+i \tilde{\Gamma}\right| \quad .
$$

\section{THE KONDO MODEL}

\section{A. Model and algebra in Liouville space}

Model. Now the RG formalism developed in the previous section is applied to the anisotropic spin- $\frac{1}{2}$ Kondo model in an external magnetic field $h_{0}>0$. In this case, we have

$$
\begin{aligned}
H_{S} & =h_{0} S^{z}, \\
g_{11^{\prime}} & =\frac{1}{2}\left\{\begin{array}{ll}
\left(J_{\alpha \alpha^{\prime}}^{i}\right)_{0} S^{i} \sigma_{\sigma \sigma^{\prime}}^{i} & \text { for } \eta=-\eta^{\prime}=+ \\
-\left(J_{\alpha^{\prime} \alpha}^{i}\right)_{0} S^{i} \sigma_{\sigma^{\prime} \sigma}^{i} & \text { for } \eta=-\eta^{\prime}=-
\end{array},\right.
\end{aligned}
$$


where $\alpha$ denotes the reservoir index, $i \in\{x, y, z\}$, and

$$
\left(J_{\alpha \alpha^{\prime}}^{x}\right)_{0}=\left(J_{\alpha \alpha^{\prime}}^{y}\right)_{0}=\left(J_{\alpha \alpha^{\prime}}^{\perp}\right)_{0} .
$$

$S^{i}$ is the $i$ component of the spin- $\frac{1}{2}$ operator of the quantum dot, $\sigma^{i}$ is a Pauli matrix, and $\left(J_{\alpha \alpha^{\prime}}^{z}\right)_{0}\left[\left(J_{\alpha \alpha^{\prime}}^{\perp}\right)_{0}\right]$ are the initial exchange couplings which correspond to processes without (with) spin flip. If we choose the reservoir states such that the exchange couplings are real, we find from hermiticity (19) that the exchange coupling matrices are symmetric,

$$
\left(J_{\alpha \alpha^{\prime}}^{z / \perp}\right)_{0}=\left(J_{\alpha^{\prime} \alpha}^{z / \perp}\right)_{0}
$$

If one derives the Kondo model via a Schrieffer-Wolff transformation from an Anderson impurity model (see, e.g., Ref. 27), one finds

$$
\left(J_{\alpha \alpha^{\prime}}^{z / \perp}\right)_{0}=2 \sqrt{x_{\alpha} x_{\alpha^{\prime}}} J_{0}^{z / \perp} \quad, \quad \sum_{\alpha} x_{\alpha}=1
$$

which is used sometimes to simplify the calculations. Furthermore, although the general formalism and many of the following formulas are also valid for an arbitrary number of reservoirs, we will finally consider the case of two reservoirs only, with $\alpha \equiv L, R \equiv \pm$, and chemical potentials given by

$$
\mu_{\alpha}=\alpha \frac{V}{2}
$$

where $V$ is the applied voltage.

Representation in Liouville space. In Liouville space, the initial Liouvillian and the initial vertex are given by

$$
\begin{aligned}
L_{S}^{(0)} & =\left[H_{S}, \cdot\right]_{-}=h_{0}\left(L^{+z}+L^{-z}\right), \\
G_{11^{\prime}}^{p p} & =\frac{1}{2}\left\{\begin{array}{cl}
\left(J_{\alpha \alpha^{\prime}}^{i}\right)_{0} L^{p i} \sigma_{\sigma \sigma^{\prime}}^{i} & \text { for } \eta=-\eta^{\prime}=+ \\
-\left(J_{\alpha^{\prime} \alpha}^{i}\right)_{0} L^{p i} \sigma_{\sigma^{\prime} \sigma}^{i} & \text { for } \eta=-\eta^{\prime}=-
\end{array},\right.
\end{aligned}
$$

where the spin superoperators $\underline{L}^{p}=\left(L^{p x}, L^{p y}, L^{p z}\right)$ are defined by their action on an arbitrary operator $A$ in Hilbert space via

$$
\underline{L}^{+} A=\underline{S} A \quad, \quad \underline{L}^{-} A=-A \underline{S} .
$$

We will now derive a closed set of basis superoperators to represent the renormalized Liouvillian and the renormalized vertices (note that this set will be more complex than the one derived in Ref. 17 for the isotropic Kondo model). Because the Hilbert space (spanned by the states $|\uparrow\rangle$ and $|\downarrow\rangle)$ is two dimensional, the Liouville space of operators acting on it is four dimensional, and the superoperators defined in this section can be represented by $4 \times 4$ matrices. This means that we need at most $4 \times 4=16$ basis superoperators.

We define the four scalar superoperators

$$
\begin{array}{ll}
L^{a}=\frac{3}{4} \cdot \mathbf{1}+\underline{L}^{+} \cdot \underline{L}^{-}, & L^{b}=\frac{1}{4} \cdot \mathbf{1}-\underline{L}^{+} \cdot \underline{L}^{-}, \\
L^{c}=\frac{1}{2} \cdot \mathbf{1}+2 L^{+z} L^{-z}, & L^{h}=L^{+z}+L^{-z},
\end{array}
$$

where $\mathbf{1}$ is the identity superoperator, and the vector superoperators

$$
\begin{aligned}
& \underline{L}^{1}=\frac{1}{2}\left(\underline{L}^{+}-\underline{L}^{-}-2 i \underline{L}^{+} \times \underline{L}^{-}\right), \\
& \underline{L}^{2}=-\frac{1}{2}\left(\underline{L}^{+}+\underline{L}^{-}\right), \\
& \underline{L}^{3}=\frac{1}{2}\left(\underline{L}^{+}-\underline{L}^{-}+2 i \underline{L}^{+} \times \underline{L}^{-}\right) .
\end{aligned}
$$

Because the Hamiltonian is not isotropic, we have to split the vector superoperators (209) into their components and consider these separately. We define

$$
L_{ \pm}^{i}=L^{i x} \pm i L^{i y} \quad \text { for } i \in\{1,2,3,+,-\}
$$

and find that we get a convenient superoperator basis if we add the set $\left\{L^{1 z}, L^{3 z}, L_{ \pm}^{1}, L_{ \pm}^{3}, L_{ \pm}^{4}, L_{ \pm}^{5}\right\}$ to the four scalar superoperators, where

$$
\begin{aligned}
& L_{ \pm}^{4}=L_{ \pm}^{2} \pm\left(L_{ \pm}^{+} L^{-z}+L^{+z} L_{ \pm}^{-}\right), \\
& L_{ \pm}^{5}=L_{ \pm}^{2} \mp\left(L_{ \pm}^{+} L^{-z}+L^{+z} L_{ \pm}^{-}\right)
\end{aligned}
$$

This set of 14 basis superoperators is sufficient to describe the anisotropic Kondo model with $J^{x}=J^{y}=J^{\perp}$, where rotational invariance around the $z$ axis holds. In the case of the fully anisotropic Kondo model with $J^{x} \neq J^{y}$, two additional superoperators

$$
\begin{gathered}
L_{++}=L_{+}^{3} L_{+}^{1}=-L_{+}^{5} L_{+}^{4} \\
L_{--}=L_{-}^{3} L_{-}^{1}=-L_{-}^{5} L_{-}^{4}
\end{gathered}
$$

have to be used, which we do not need in the following.

We note the properties

$$
\begin{aligned}
& \operatorname{Tr}_{S} L^{\chi}=0 \quad, \quad \text { for } \chi=a, c, h, 3 z, \\
& \operatorname{Tr}_{S} L_{ \pm}^{\chi}=0 \quad, \quad \text { for } \chi=3,4,5, \\
& \operatorname{Tr}_{S} L^{b}, \operatorname{Tr}_{S} L^{1 z}, \operatorname{Tr}_{S} L_{ \pm}^{1} \neq 0,
\end{aligned}
$$

together with the transformation under the $c$ transform (66)

$$
\begin{aligned}
& \left(L^{\chi}\right)^{c}=L^{\chi}, \quad \text { for } \chi=a, b, c, 1 z, 3 z \\
& \left(L^{h}\right)^{c}=-L^{h}, \\
& \left(L_{ \pm}^{\chi}\right)^{c}=L_{\mp}^{\chi}, \quad \text { for } \chi=1,3, \\
& \left(L_{ \pm}^{\chi}\right)^{c}=-L_{\mp}^{\chi}, \quad \text { for } \chi=4,5 .
\end{aligned}
$$

Using spin rotational invariance around the $z$ axis and spin conservation, together with the properties (215)(217) and $\operatorname{Tr}_{S} L_{S}(E, \omega)=0$ [see Eq. (99)], we find that the Liouvillian and the current kernel can be represented as (in each step of the RG)

$$
\begin{aligned}
L_{S}(E, \omega) & =h(E, \omega) L^{h}-i \Gamma^{a}(E, \omega) L^{a} \\
& -i \Gamma^{c}(E, \omega) L^{c}-i \Gamma^{3 z}(E, \omega) L^{3 z} \\
\Sigma_{\gamma}(E, \omega) & =i \Gamma_{\gamma}^{b}(E, \omega) L^{b}+i \Gamma_{\gamma}^{1 z}(E, \omega) L^{1 z},
\end{aligned}
$$

where as usual we always assume implicitly that the $\operatorname{Tr}_{S}$ is acting from the left when we consider the current kernel or the current vertex

$$
\Sigma_{\gamma} \equiv \operatorname{Tr}_{S} \Sigma_{\gamma} \quad, \quad \bar{I}_{12}^{\gamma} \equiv \operatorname{Tr}_{S} \bar{I}_{12}^{\gamma}
$$


From Eqs. (101), (102), (218), and (219), we get the following transformation under complex conjugation:

$$
\begin{aligned}
\Gamma^{\chi}(E, \omega)^{*} & =\Gamma^{\chi}(-E, \omega), \text { for } \chi=a, c, 3 z, \\
\Gamma_{\gamma}^{\chi}(E, \omega)^{*} & =\Gamma_{\gamma}^{\chi}(-E, \omega), \text { for } \chi=b, 1 z, \\
h(E, \omega)^{*} & =h(-E, \omega) .
\end{aligned}
$$

The various terms in Eq. (222) can be interpreted if one analyzes the spectral properties of the renormalized Liouvillian. $\mathrm{Ł}_{S}(E, \omega)$ has four eigenvalues,

$$
\begin{aligned}
\lambda_{0}(E, \omega) & =0, \\
\lambda_{1}(E, \omega) & =-i \Gamma^{a}(E, \omega), \\
\lambda_{ \pm}(E, \omega) & = \pm h(E, \omega)-i\left(\Gamma^{a}+\Gamma^{c}\right)(E, \omega) .
\end{aligned}
$$

$\lambda_{0}$ corresponds to the stationary state, $\lambda_{1}$ describes the relaxation mode, and $\lambda_{ \pm}$correspond to the two dephasing modes. The projectors $P_{i}(E, \omega)=$ $\mid x_{i}(E, \omega\rangle\left\langle\bar{x}_{i}(E, \omega)\right|$ onto the four eigenspaces are given by

$$
\begin{aligned}
& P_{0}(E, \omega)=L^{b}-\frac{\Gamma^{3 z}(E, \omega)}{\Gamma^{a}(E, \omega)} L^{3 z} \\
& P_{1}(E, \omega)=L^{a}-L^{c}+\frac{\Gamma^{3 z}(E, \omega)}{\Gamma^{a}(E, \omega)} L^{3 z} \\
& P_{ \pm}(E, \omega)=\frac{1}{2}\left(L^{c} \pm L^{h}\right)
\end{aligned}
$$

and the right and left eigenvectors follow from

$$
\begin{aligned}
\left\langle\sigma \sigma^{\prime} \mid x_{0}(E, \omega)\right\rangle & =\delta_{\sigma \sigma^{\prime}}\left(\frac{1}{2}-\sigma \frac{\Gamma^{3 z}(E, \omega)}{2 \Gamma^{a}(E, \omega)}\right), \\
\left\langle\sigma \sigma^{\prime} \mid x_{1}(E, \omega)\right\rangle & =\delta_{\sigma \sigma^{\prime}} \sigma \\
\left\langle\sigma \sigma^{\prime} \mid x_{ \pm}(E, \omega)\right\rangle & =\delta_{\sigma,-\sigma^{\prime}} \delta_{\sigma \pm} \\
\left\langle\bar{x}_{0}(E, \omega) \mid \sigma \sigma^{\prime}\right\rangle & =\delta_{\sigma \sigma^{\prime}}, \\
\left\langle\bar{x}_{1}(E, \omega) \mid \sigma \sigma^{\prime}\right\rangle & =\delta_{\sigma \sigma^{\prime}}\left(\frac{1}{2} \sigma+\frac{\Gamma^{3 z}(E, \omega)}{2 \Gamma^{a}(E, \omega)}\right), \\
\left\langle\bar{x}_{ \pm}(E, \omega) \mid \sigma \sigma^{\prime}\right\rangle & =\delta_{\sigma,-\sigma^{\prime}} \delta_{\sigma \pm} .
\end{aligned}
$$

According to Eq. (44), the eigenvector $\left|x_{0}\left(0,0^{+}\right)\right\rangle$corresponds to the stationary state. Therefore, we get

$$
\rho_{S}^{s t}=\frac{1}{2} \mathbf{1}+2 M S^{z} \quad, \quad M=-\frac{\Gamma^{3 z}}{2 \Gamma^{a}},
$$

where $M$ denotes the magnetization, which is related to $\Gamma^{3 z}$ (if no argument is written, we implicitly assume $E=$ 0 and $\left.\omega=0^{+}\right)$.

The stationary current follows from Eqs. (45) and (223) as

$$
\begin{aligned}
\left\langle I^{\gamma}\right\rangle^{s t} & =-i \operatorname{Tr}_{S} \Sigma_{\gamma} \rho_{S}^{s t} \\
& =\Gamma_{\gamma}^{b} \operatorname{Tr}_{S} L^{b} \rho_{S}^{s t}+\Gamma_{\gamma}^{1 z} \operatorname{Tr}_{S} L^{1 z} \rho_{S}^{s t},
\end{aligned}
$$

which, by using Eq. (230) together with $\frac{1}{2} \operatorname{Tr}_{S} L^{b} \mathbf{1}=$ $\operatorname{Tr}_{S} L^{1 z} S^{z}=1$ and $\operatorname{Tr}_{S} L^{b} S^{z}=\operatorname{Tr}_{S} L^{1 z} \mathbf{1}=0$, gives

$$
\left\langle I^{\gamma}\right\rangle^{s t}=\Gamma_{\gamma}^{b}+2 M \Gamma_{\gamma}^{1 z} .
$$

To represent the vertices, we define the reservoir spin matrices

$$
\sigma_{ \pm}^{z}=\frac{1}{2}\left(1 \pm \sigma^{z}\right) \quad, \quad \sigma_{ \pm}=\frac{1}{2}\left(\sigma_{x} \pm i \sigma_{y}\right) \quad,
$$

and the following operators in combined reservoir spin space and Liouville space of the dot

$$
\begin{aligned}
& \hat{L}_{ \pm}^{\chi}=L^{\chi} \sigma_{ \pm}^{z}, \text { for } \chi=a, b, c, h, 1 z, 3 z, \\
& \hat{L}_{ \pm}^{\chi}=L_{ \pm}^{\chi} \sigma_{\mp}, \text { for } \chi=1,3,4,5 .
\end{aligned}
$$

Using spin rotational invariance around the $z$ axis, together with the properties (100) and (215)-(217), the renormalized vertices can be represented as (for $\eta_{1}=$ $\left.-\eta_{2}=+\right)$

$$
\begin{aligned}
& \left.\bar{G}_{12}\left(E, \omega, \omega_{1}, \omega_{2}\right)\right|_{\eta_{1}=-\eta_{2}=+}= \\
& \quad=\sum_{s= \pm} \sum_{\substack{\chi=a, c, h, 3 z, 3,4,5}} \bar{G}^{\chi s}\left(E, \omega, \omega_{1}, \omega_{2}\right) \hat{L}_{s}^{\chi}, \\
& \left.\quad \bar{I}_{12}^{\gamma}\left(E, \omega, \omega_{1}, \omega_{2}\right)\right|_{\eta_{1}=-\eta_{2}=+}= \\
& \quad \sum_{s= \pm} \sum_{\chi=b, 1 z, 1} \bar{I}^{\gamma \chi s}\left(E, \omega, \omega_{1}, \omega_{2}\right) \hat{L}_{s}^{\chi} .
\end{aligned}
$$

In these equations, we have used a compact matrix notation in the reservoir indices $\left(\alpha_{1}, \alpha_{2}\right)$ and the reservoir spin indices $\left(\sigma_{1}, \sigma_{2}\right)$. Whereas $\bar{G}^{\chi s} \equiv\left(\bar{G}_{\alpha_{1} \alpha_{2}}^{\chi s}\right)$ and $\bar{I}^{\gamma \chi s} \equiv\left(\bar{I}_{\alpha_{1} \alpha_{2}}^{\gamma \chi s}\right)$ denote matrices in the reservoir indices, the quantities $\hat{L}_{s}^{\chi}$ are matrices in the reservoir spin indices according to definitions (233) and (234). Again, we note that Eq. (236) holds only if the trace $\operatorname{Tr}_{S}$ is taken from the left. For $\eta_{1}=-\eta_{2}=-$, we use the antisymmetry properties (97) and (98), and obtain from Eqs. (235) and (236)

$$
\begin{aligned}
& \left.\bar{G}_{12}\left(E, \omega, \omega_{1}, \omega_{2}\right)\right|_{\eta_{1}=-\eta_{2}=-}= \\
& =-\sum_{s= \pm} \sum_{\substack{\chi=a, c, h, 3 z, 3,4,5}} \bar{G}^{\chi s}\left(E, \omega, \omega_{2}, \omega_{1}\right)^{T}\left(\hat{L}_{s}^{\chi}\right)^{T}, \\
& \left.\bar{I}_{12}^{\gamma}\left(E, \omega, \omega_{1}, \omega_{2}\right)\right|_{\eta_{1}=-\eta_{2}=-}= \\
& \quad=-\sum_{s= \pm} \sum_{\chi=b, 1 z, 1} \bar{I}^{\gamma \chi s}\left(E, \omega, \omega_{2}, \omega_{1}\right)^{T}\left(\hat{L}_{s}^{\chi}\right)^{T}
\end{aligned}
$$

where $(\ldots)^{T}$ denotes the transpose only with respect to the reservoir indices or the reservoir spin indices

$$
\begin{aligned}
\left(\bar{G}^{\chi s}\right)_{\alpha \alpha^{\prime}}^{T} & =\bar{G}_{\alpha^{\prime} \alpha}^{\chi s} \quad, \quad\left(\bar{I}^{\gamma \chi s}\right)_{\alpha \alpha^{\prime}}^{T}=\bar{I}_{\alpha^{\prime} \alpha}^{\gamma \chi s}, \\
\left(\hat{L}_{s}^{\chi}\right)_{\sigma \sigma^{\prime}}^{T} & =\left(\hat{L}_{s}^{\chi}\right)_{\sigma^{\prime} \sigma} .
\end{aligned}
$$

Using the properties (103) and (104) together with Eqs. (218)-(221), we obtain the symmetry relations

$$
\begin{gathered}
\bar{G}_{\alpha \alpha^{\prime}}^{\chi s}\left(E, \omega, \omega_{1}, \omega_{2}\right)^{*}=-\bar{G}_{\alpha^{\prime} \alpha}^{\chi s}\left(-E, \omega, \omega_{2}, \omega_{1}\right) \\
\text { for } \chi=a, b, c, 1 z, 3 z, \\
\bar{G}_{\alpha \alpha^{\prime}}^{h s}\left(E, \omega, \omega_{1}, \omega_{2}\right)^{*}=\bar{G}_{\alpha^{\prime} \alpha}^{h s}\left(-E, \omega, \omega_{2}, \omega_{1}\right), \\
\bar{G}_{\alpha \alpha^{\prime}}^{1 / 3, s}\left(E, \omega, \omega_{1}, \omega_{2}\right)^{*}=-\bar{G}_{\alpha^{\prime} \alpha}^{1 / 3,-s}\left(-E, \omega, \omega_{2}, \omega_{1}\right), \\
\bar{G}_{\alpha \alpha^{\prime}}^{4 / 5, s}\left(E, \omega, \omega_{1}, \omega_{2}\right)^{*}=\bar{G}_{\alpha^{\prime} \alpha}^{4 / 5,-s}\left(-E, \omega, \omega_{2}, \omega_{1}\right),
\end{gathered}
$$


and the same for $\bar{G} \rightarrow \bar{I}^{\gamma}$.

Algebra. The results of a multiplication of two of the operators $\hat{L}_{ \pm}^{\chi}$ are summarized in the following table (all products not shown are zero):

\begin{tabular}{c||cccc|cc|cccc} 
& $\hat{L}_{ \pm}^{a}$ & $\hat{L}_{ \pm}^{b}$ & $\hat{L}_{ \pm}^{c}$ & $\hat{L}_{ \pm}^{h}$ & $\hat{L}_{ \pm}^{1 z}$ & $\hat{L}_{ \pm}^{3 z}$ & $\hat{L}_{\mp}^{1}$ & $\hat{L}_{\mp}^{3}$ & $\hat{L}_{\mp}^{4}$ & $\hat{L}_{\mp}^{5}$ \\
\hline \hline$\hat{L}_{ \pm}^{a}$ & $\hat{L}_{ \pm}^{a}$ & 0 & $\hat{L}_{ \pm}^{c}$ & $\hat{L}_{ \pm}^{h}$ & 0 & $\hat{L}_{ \pm}^{3 z}$ & 0 & $\hat{L}_{\mp}^{3}$ & $\hat{L}_{\mp}^{4}$ & $\hat{L}_{\mp}^{5}$ \\
$\hat{L}_{ \pm}^{b}$ & 0 & $\hat{L}_{ \pm}^{b}$ & 0 & 0 & $\hat{L}_{ \pm}^{1 z}$ & 0 & $\hat{L}_{\mp}^{1}$ & 0 & 0 & 0 \\
$\hat{L}_{ \pm}^{c}$ & $\hat{L}_{ \pm}^{c}$ & 0 & $\hat{L}_{ \pm}^{c}$ & $\hat{L}_{ \pm}^{h}$ & 0 & 0 & 0 & $\hat{L}_{\mp}^{3}$ & 0 & $\hat{L}_{\mp}^{5}$ \\
$\hat{L}_{ \pm}^{h}$ & $\hat{L}_{ \pm}^{h}$ & 0 & $\hat{L}_{ \pm}^{h}$ & $\hat{L}_{ \pm}^{c}$ & 0 & 0 & 0 & $\mp \hat{L}_{\mp}^{3}$ & 0 & $\mp \hat{L}_{\mp}^{5}$ \\
\hline$\hat{L}_{ \pm}^{1 z}$ & $\hat{L}_{ \pm}^{1 z}$ & 0 & 0 & 0 & 0 & $\hat{L}_{ \pm}^{b}$ & 0 & 0 & $\pm \hat{L}_{\mp}^{1}$ & 0 \\
$\hat{L}_{ \pm}^{3 z}$ & 0 & $\hat{L}_{ \pm}^{3 z}$ & 0 & 0 & $\hat{L}_{ \pm}^{a}-\hat{L}_{ \pm}^{c}$ & 0 & $\pm \hat{L}_{\mp}^{4}$ & 0 & 0 & 0 \\
\hline$\hat{L}_{ \pm}^{1}$ & $\hat{L}_{ \pm}^{1}$ & 0 & $\hat{L}_{ \pm}^{1}$ & $\mp \hat{L}_{ \pm}^{1}$ & 0 & 0 & 0 & $2 \hat{L}_{\mp}^{b}$ & 0 & $\mp 2 \hat{L}_{\mp}^{1 z}$ \\
$\hat{L}_{ \pm}^{3}$ & 0 & $\hat{L}_{ \pm}^{3}$ & 0 & 0 & $\pm \hat{L}_{ \pm}^{5}$ & 0 & $\hat{L}_{\mp}^{c} \pm \hat{L}_{\mp}^{h}$ & 0 & 0 & 0 \\
$\hat{L}_{ \pm}^{4}$ & $\hat{L}_{ \pm}^{4}$ & 0 & $\hat{L}_{ \pm}^{4}$ & $\mp \hat{L}_{ \pm}^{4}$ & 0 & 0 & 0 & $\mp 2 \hat{L}_{\mp}^{3 z}$ & 0 & $2 \hat{L}_{\mp}^{a}-2 \hat{L}_{\mp}^{c}$ \\
$\hat{L}_{ \pm}^{5}$ & $\hat{L}_{ \pm}^{5}$ & 0 & 0 & 0 & 0 & $\pm \hat{L}_{ \pm}^{3}$ & 0 & 0 & $\hat{L}_{\mp}^{c} \pm \hat{L}_{\mp}^{h}$ & 0
\end{tabular}

Note that, according to definitions (233) and (234), the operators $\hat{L}_{ \pm}^{\chi}$ are matrices in the reservoir spin indices, where each matrix element is a superoperator in the Liouville space of the dot. The same algebra holds without the reservoir spin indices if one replaces

$$
\begin{aligned}
& \hat{L}_{ \pm}^{\chi} \rightarrow L^{\chi} \text { for } \chi=a, b, c, h, 1 z, 3 z \\
& \hat{L}_{ \pm}^{\chi} \rightarrow L_{ \pm}^{\chi} \text { for } \chi=1,3,4,5,
\end{aligned}
$$

in Eq. (241), with the only difference that also some products not shown are nonzero [these are the products $L_{ \pm}^{3} L_{ \pm}^{1}=-L_{ \pm}^{5} L_{ \pm}^{4}$ defining the basis superoperators $L_{ \pm \pm}$ according to Eqs. (213) and (214), which are not needed for our case of rotational invariance around the $z$ axis]. Furthermore, we note that the algebra (241) is also not changed if we replace

$$
\begin{aligned}
\hat{L}_{ \pm}^{\chi} & \rightarrow\left(\hat{L}_{ \pm}^{\chi}\right)^{T} \text { for } \chi=a, b, c, h, 1 z, 3 z, \\
\hat{L}_{ \pm}^{\chi} & \rightarrow\left(\hat{L}_{\mp}^{\chi}\right)^{T} \text { for } \chi=1,3,4,5, \\
\pm & \rightarrow \mp \text { for all sign factors }
\end{aligned}
$$

which turns out to be very helpful to consider the two cases $\eta= \pm$ for creation and annihilation operators, see the representations (237) and (238).

Finally, we note that the spin matrices (232) fulfil the algebra (all products not shown are zero)

$$
\begin{array}{c||c|c} 
& \sigma_{ \pm}^{z} & \sigma_{ \pm} \\
\hline \hline \sigma_{ \pm}^{z} & \sigma_{ \pm}^{z} & \sigma_{ \pm} \\
\sigma_{\mp} & \sigma_{\mp} & \sigma_{\mp}^{z}
\end{array}
$$

and the same holds if one replaces

$$
\sigma_{ \pm}^{z} \rightarrow\left(\sigma_{ \pm}^{z}\right)^{T} \quad, \quad \sigma_{ \pm} \rightarrow\left(\sigma_{\mp}\right)^{T}
$$

\section{B. Two-loop analysis}

\section{RG above $\Lambda_{c}$}

Initial values. We start with the determination of the initial values of the Liouvillian and the vertices together with their values after the first RG step, given by Eqs. (73)-(75).

The initial values for the Liouvillian $L_{S}^{(0)}$ and the vertices $\bar{G}_{11^{\prime}}^{(1)}$ and $\tilde{G}_{11^{\prime}}^{(1)}$ follow from Eqs. (205) and (206) as

$$
\begin{aligned}
L_{S}^{(0)} & =\left[H_{S}, \cdot\right]_{-}=h_{0} L^{h}, \\
\bar{G}_{11^{\prime}}^{(1)} & =\sum_{p} G_{11^{\prime}}^{p p}=-\hat{J}_{0}^{i} L^{2 i} \sigma^{i}, \\
\tilde{G}_{11^{\prime}}^{(1)} & =\sum_{p} p G_{11^{\prime}}^{p p}=-\frac{1}{2} \hat{J}_{0}^{i}\left(L^{1 i}+L^{3 i}\right) \sigma^{i},
\end{aligned}
$$

where we have taken $\eta=-\eta^{\prime}=+$ and used a matrix notation $\hat{J}_{0}^{i} \equiv\left[\left(J_{0}^{i}\right)_{\alpha \alpha^{\prime}}\right]$ for the exchange couplings in the reservoir indices. Inserting the various definitions of the basis superoperators, we find the following representations for $\eta=-\eta^{\prime}=+$ :

$$
\begin{aligned}
\bar{G}_{11^{\prime}}^{(1)} & =\frac{1}{2} \hat{J}_{0}^{z} s \hat{L}_{s}^{h}-\frac{1}{2} \hat{J}_{0}^{\perp}\left(\hat{L}_{s}^{4}+\hat{L}_{s}^{5}\right) \\
\tilde{G}_{11^{\prime}}^{(1)} & =\frac{1}{2} \hat{J}_{0}^{z} s\left(\hat{L}_{s}^{1 z}+\hat{L}_{s}^{3 z}\right)+\frac{1}{2} \hat{J}_{0}^{\perp}\left(\hat{L}_{s}^{1}+\hat{L}_{s}^{3}\right)
\end{aligned}
$$

where we sum implicitly over $s= \pm$ on the r.h.s. According to Eq. (41), the initial current vertex is given by $\bar{I}_{11^{\prime}}^{\gamma(1)}=c_{11^{\prime}}^{\gamma} \tilde{G}_{11^{\prime}}^{(1)}$. Using Eq. (250), we obtain for $\eta=-\eta^{\prime}=+$

$$
\bar{I}_{11^{\prime}}^{\gamma(1)}=\frac{1}{2} \hat{J}_{0}^{\gamma z} s \hat{L}_{s}^{1 z}+\frac{1}{2} \hat{J}_{0}^{\gamma \perp} \hat{L}_{s}^{1}
$$


where

$$
\left(J_{\alpha \alpha^{\prime}}^{\gamma, z / \perp}\right)_{0}=c_{\alpha \alpha^{\prime}}^{\gamma}\left(J_{\alpha \alpha^{\prime}}^{z / \perp}\right)_{0} \quad, \quad c_{\alpha \alpha^{\prime}}^{\gamma}=-\frac{1}{2}\left(\delta_{\gamma \alpha}-\delta_{\gamma \alpha^{\prime}}\right) .
$$

We have left out the components $3 z$ and 3 in Eq. (251) because we assume implicitly that the $\operatorname{Tr}_{S}$ acts from the left on the current vertex.

To calculate the second-order contributions (73)- 75 from the first $\mathrm{RG}$ step, we use the following identities for any two superoperators $A_{11^{\prime}}=A^{\chi s} \hat{L}_{s}^{\chi}$ and $B_{11^{\prime}}=$ $B^{\chi s} \hat{L}_{s}^{\chi}$, which can all be proven easily by summing over the two possibilities $\eta_{i}= \pm$ :

$$
\begin{aligned}
& A_{11^{\prime}} B_{\overline{1}^{\prime} \overline{1}}=2\left(\operatorname{Tr}_{\alpha} A^{\chi s} B^{\chi^{\prime} s^{\prime}}\right)\left(\operatorname{Tr}_{\sigma} \hat{L}_{s}^{\chi} \hat{L}_{s^{\prime}}^{\chi^{\prime}}\right), \\
& A_{11^{\prime}}\left(E_{11^{\prime}}-L_{S}^{(0)}\right) B_{\overline{1}^{\prime} \overline{1}}= \\
& 2 A_{\alpha \alpha^{\prime}}^{\chi s} B_{\alpha^{\prime} \alpha}^{\chi^{\prime} s^{\prime}}\left(\operatorname{Tr}_{\sigma} \hat{L}_{s}^{\chi}\left(E_{\alpha \alpha^{\prime}}-h_{0} L^{h}\right) \hat{L}_{s^{\prime}}^{\chi^{\prime}}\right),
\end{aligned}
$$

with $E_{\alpha \alpha^{\prime}}=E+\mu_{\alpha}-\mu_{\alpha^{\prime}}$. Here, $\operatorname{Tr}_{\alpha}$ and $\operatorname{Tr}_{\sigma}$ denote the trace with respect to the reservoir indices and the reservoir spin indices, respectively. Applying these identities to Eqs. (73)- (75), inserting the results (249)-(251) for the vertices, and applying the algebra of the basis superoperators, we find after some straightforward calculation the following result for the Liouvillian and the current kernel after the first RG step:

$$
\begin{aligned}
L_{S}^{a}(E)= & -i \frac{\pi^{2}}{16}(2 D+i E)\left(\operatorname{Tr} \hat{J}_{0}^{\perp} \hat{J}_{0}^{\perp}\right) L^{a} \\
& -i \frac{\pi^{2}}{32}(2 D+i E)\left(\operatorname{Tr}\left(\hat{J}_{0}^{z} \hat{J}_{0}^{z}-\hat{J}_{0}^{\perp} \hat{J}_{0}^{\perp}\right)\right) L^{c} \\
& +h_{0}\left(1-\frac{\pi^{2}}{32}\right)\left(\operatorname{Tr} \hat{J}_{0}^{z} \hat{J}_{0}^{z}\right) L^{h} \\
& -i \frac{\pi}{2} h_{0}\left(\operatorname{Tr} \hat{J}_{0}^{\perp} \hat{J}_{0}^{\perp}\right) L^{3 z} \\
\Sigma_{\gamma}^{a}(E)= & i \frac{\pi}{4} \delta_{\alpha \gamma}\left(\mu_{\alpha}-\mu_{\alpha^{\prime}}\right)\left(\left(J_{\alpha \alpha^{\prime}}^{z}\right)^{2}+2\left(J_{\alpha \alpha^{\prime}}^{\perp}\right)^{2}\right)_{0} L^{b}
\end{aligned}
$$

Thereby, the terms proportional to the band width cancel with terms generated by the second RG step, see Eq. (163). Therefore, we can omit them together with the real terms of second order in $J$ [which are not the leading order ones in this order, see the discussion before Eq. (192)]. Thus, we are left with the following inital condition to solve the RG equations (158) and (159):

$$
\begin{aligned}
& \left.L_{S}^{(2 b)}\right|_{\Lambda=\Lambda_{0}}=-i \frac{\pi}{2} h_{0}\left(\operatorname{Tr} \hat{J}_{0}^{\perp} \hat{J}_{0}^{\perp}\right) L^{3 z}, \\
& \left.\Sigma_{\gamma}^{(2 b)}\right|_{\Lambda=\Lambda_{0}}= \\
& \quad=-i \frac{\pi}{4} c_{\alpha \alpha^{\prime}}^{\gamma}\left(\mu_{\alpha}-\mu_{\alpha^{\prime}}\right)\left(\left(J_{\alpha \alpha^{\prime}}^{z}\right)^{2}+2\left(J_{\alpha \alpha^{\prime}}^{\perp}\right)^{2}\right)_{0} L^{b}, \\
& \left.Z^{(2 b)}\right|_{\Lambda=\Lambda_{0}}=\left.Z_{\gamma}^{(2 b)}\right|_{\Lambda=\Lambda_{0}}=0 .
\end{aligned}
$$

We now calculate the form of the vertex (75) after the first RG step, which leads to the initial condition for the vertices $\bar{G}_{11^{\prime}}^{\left(2 a_{1}\right)}$ and $\bar{I}_{11^{\prime}}^{\gamma\left(2 a_{1}\right)}$, given by Eq. (145). We use the following identity, valid for $\eta=-\eta^{\prime}=+$ :

$$
\begin{aligned}
& A_{12} B_{\overline{2} 1^{\prime}}-\left(1 \leftrightarrow 1^{\prime}\right)= \\
& =A^{\chi s} B^{\chi^{\prime} s^{\prime}} \hat{L}_{s}^{\chi} \hat{L}_{s^{\prime}}^{\chi^{\prime}}-B^{\chi^{\prime} s^{\prime}} A^{\chi s}\left(\left(\hat{L}_{s}^{\chi}\right)^{T}\left(\hat{L}_{s^{\prime}}^{\chi^{\prime}}\right)^{T}\right)^{T} .
\end{aligned}
$$

Applying this property to Eq. (145), inserting Eqs. (249)(251), and applying the algebra of the basis superoperators, we find after some straightforward calculation for $\eta=-\eta^{\prime}=+$

$$
\begin{aligned}
\left.\bar{G}_{11^{\prime}}^{\left(2 a_{1}\right)}\right|_{\Lambda=\Lambda_{0}}= & \frac{\pi}{2} \hat{J}_{0}^{\perp} \hat{J}_{0}^{\perp} s \hat{L}_{s}^{3 z} \\
& +\frac{\pi}{4}\left(\hat{J}_{0}^{z} \hat{J}_{0}^{\perp}+\hat{J}_{0}^{\perp} \hat{J}_{0}^{z}\right) \hat{L}_{s}^{3}, \\
\left.\bar{I}_{11^{\prime}}^{\gamma\left(2 a_{1}\right)}\right|_{\Lambda=\Lambda_{0}}= & -\frac{\pi}{8}\left(\hat{J}_{0}^{\gamma z} \hat{J}_{0}^{z}-\hat{J}_{0}^{z} \hat{J}_{0}^{\gamma z}+\right. \\
& \left.+2 \hat{J}_{0}^{\gamma \perp} \hat{J}_{0}^{\perp}-2 \hat{J}_{0}^{\perp} \hat{J}_{0}^{\gamma \perp}\right) \hat{L}_{s}^{b} .
\end{aligned}
$$

Vertices in first order. The RG equations for the lowest order vertices $\bar{G}_{11^{\prime}}^{(1)}$ and $\bar{I}_{11^{\prime}}^{(1)}$ are given by Eqs. (115) and (116) with initial conditions (249) and (251). Using Eq. (260), one finds after some algebra that the initial form of the leading order vertices is preserved, i.e., for $\eta=-\eta^{\prime}=+$

$$
\begin{aligned}
\bar{G}_{11^{\prime}}^{(1)} & =\frac{1}{2} \hat{J}^{z} s \hat{L}_{s}^{h}-\frac{1}{2} \hat{J}^{\perp}\left(\hat{L}_{s}^{4}+\hat{L}_{s}^{5}\right), \\
\bar{I}_{11^{\prime}}^{\gamma(1)} & =\frac{1}{2} \hat{J}^{\gamma z} s \hat{L}_{s}^{1 z}+\frac{1}{2} \hat{J}^{\gamma \perp} \hat{L}_{s}^{1},
\end{aligned}
$$

with

$$
J_{\alpha \alpha^{\prime}}^{\gamma, z / \perp}=c_{\alpha \alpha^{\prime}}^{\gamma} J_{\alpha \alpha^{\prime}}^{z / \perp} \quad, \quad c_{\alpha \alpha^{\prime}}^{\gamma}=-\frac{1}{2}\left(\delta_{\gamma \alpha}-\delta_{\gamma \alpha^{\prime}}\right),
$$

provided that the renormalized exchange coupling matrices fulfil the poor man scaling RG equations

$$
\frac{d}{d l} \hat{J}^{z}=\left(\hat{J}^{\perp}\right)^{2} \quad, \quad \frac{d}{d l} \hat{J}^{\perp}=\frac{1}{2}\left(\hat{J}^{z} \hat{J}^{\perp}+\hat{J}^{\perp} \hat{J}^{z}\right)
$$

where $l=\ln \Lambda_{0} / \Lambda$ is the dimensionless flow parameter and $\left.\hat{J}^{z / \perp}\right|_{\Lambda=\Lambda_{0}}=\hat{J}_{0}^{z / \perp}$ is the initial condition.

The poor man scaling equations have the invariant

$$
(2 c)^{2} \equiv \operatorname{Tr}\left(\hat{J}^{z} \hat{J}^{z}-\hat{J}^{\perp} \hat{J}^{\perp}\right)=\text { const } .
$$

They can be solved easily if one assumes the form 203 for the initial couplings. In this case, we have the same form for the renormalized couplings,

$$
J_{\alpha \alpha^{\prime}}^{z / \perp}=2 \sqrt{x_{\alpha} x_{\alpha^{\prime}}} J^{z / \perp} \quad, \quad \sum_{\alpha} x_{\alpha}=1
$$

and the two exchange couplings $J^{z}$ and $J^{\perp}$ fulfil the RG equations

$$
\frac{d}{d l} J^{z}=2\left(J^{\perp}\right)^{2} \quad, \quad \frac{d}{d l} J^{\perp}=2 J^{z} J^{\perp} .
$$


These RG equations have two invariants

$$
c^{2} \equiv\left(J^{z}\right)^{2}-\left(J^{\perp}\right)^{2} \quad, \quad T_{K} \equiv \Lambda\left(\frac{J^{z}-c}{J^{z}+c}\right)^{\frac{1}{4 c}}
$$

where $c=i \sqrt{\left|\left(J^{z}\right)^{2}-\left(J^{\perp}\right)^{2}\right|}$ for $\left|J^{z}\right|<\left|J^{\perp}\right| . T_{K}$ denotes the Kondo temperature, which can also be written as

$$
T_{K}=\Lambda e^{-\frac{1}{2 J^{z}} \frac{1}{2 \delta} \ln \frac{1+\delta}{1-\delta}}
$$

with

$$
\delta=\operatorname{sign}\left(J^{z}\right) \sqrt{1-\left(\frac{J^{\perp}}{J^{z}}\right)^{2}},
$$

and, for $\left|J^{z}\right|<\left|J^{\perp}\right|$ [where $\left.\delta=i \operatorname{sign}\left(J^{z}\right)|\delta|\right]$, the imaginary part of the logarithm is defined such that $-2 \pi<\ln (z)<0$ (such that $T_{K}$ does not jump during the RG flow and is exponentially small). Thus, for $\left|J^{z}\right|<\left|J^{\perp}\right|$, we obtain

$$
T_{K}=\Lambda e^{-\frac{1}{2 J^{z}|\delta|}\left(\arctan |\delta|+\frac{\pi}{2}\left(\operatorname{sign}\left(J^{z}\right)-1\right)\right)} .
$$

For the isotropic case $\left(J^{z}=J^{\perp}=J\right)$, we get the usual form $T_{K}=\Lambda e^{-\frac{1}{2 J}}$. In terms of the invariants, the solution of Eq. (269) can be written as

$$
J^{z}=c \frac{1+\left(\frac{T_{K}}{\Lambda}\right)^{4 c}}{1-\left(\frac{T_{K}}{\Lambda}\right)^{4 c}} \quad, \quad J^{\perp}=2 c \frac{\left(\frac{T_{K}}{\Lambda}\right)^{2 c}}{1-\left(\frac{T_{K}}{\Lambda}\right)^{4 c}}
$$

In the scaling limit $J_{0}^{z}, J_{0}^{\perp} \rightarrow 0$ and $\Lambda_{0} \rightarrow \infty$, such that $\frac{J_{0}^{z}}{J_{0}^{\perp}}=$ const and $T_{K}=$ const (which is possible for $J^{z}>0$ or $\left|J^{z}\right|<\left|J^{\perp}\right|$ ), we obtain the well-known isotropic form

$$
J^{z}=J^{\perp}=\frac{1}{2 \ln \frac{\Lambda}{T_{K}}}
$$

with $T_{K}$ given by Eq. (271) (where the anisotropy of the initial exchange couplings still enters).

Liouvillian in first order. To determine $L_{S}^{(1)}(E, \omega)$ from Eq. (129), we use the solution (263) for $\bar{G}_{11^{\prime}}^{(1)}$ together with the identities (253) and (254) to evaluate the r.h.s. of the RG equations (130) and (131). We obtain

$$
\begin{aligned}
\frac{d}{d l} L_{S}^{(1)} & =\frac{1}{2}\left(\operatorname{Tr} \hat{J}^{z} \hat{J}^{z}\right) h_{0} L^{h}, \\
\frac{d}{d l} Z^{(1)} & =\left(\operatorname{Tr} \hat{J}^{\perp} \hat{J}^{\perp}\right) L^{a}+2 c^{2} L^{c},
\end{aligned}
$$

with the invariant $c$ given by Eq. (267). Using $\operatorname{Tr} \hat{J}^{z} \hat{J}^{z}=$ $\operatorname{Tr} \hat{J}^{\perp} \hat{J}^{\perp}+4 c^{2}$ and $\hat{J}^{\perp} \hat{J}^{\perp}=\frac{d}{d l} \hat{J}^{z}$ according to Eq. (266), we find the solution (note that the inital conditions are zero in first order in $J$ )

$$
\begin{aligned}
L_{S}^{(1)}(E, \omega) & =L_{S}^{(1)}-(E+i \omega) Z^{(1)}, \\
L_{S}^{(1)} & =\frac{1}{2} \operatorname{Tr}\left(\hat{J}^{z}-\hat{J}_{0}^{z}\right) h_{0} L^{h}+2 c^{2} l h_{0} L^{h} \\
Z^{(1)} & =\operatorname{Tr}\left(\hat{J}^{z}-\hat{J}_{0}^{z}\right) L^{a}+2 c^{2} l L^{c},
\end{aligned}
$$

where the flow parameter $l=\ln \frac{\Lambda_{0}}{\Lambda}=\ln \frac{\Lambda_{0}}{T_{K}}-\ln \frac{\Lambda}{T_{K}}$ can be expressed in terms of the exchange couplings by using Eq. (270):

$$
l=\frac{1}{4 c}\left(\ln \frac{J^{z}-c}{J^{z}+c}-\ln \frac{J_{0}^{z}-c}{J_{0}^{z}+c}\right) .
$$

Whereas the logarithms in this formula are pure anisotropy terms depending on the ratios of the exchange couplings, the prefactor $\frac{1}{4 c}$ gives $l \sim O(1 / J)$, i.e., the second terms $\sim c^{2} l$ on the r.h.s. of Eqs. (279) and (280) are of the same order $O(J)$ as the first terms.

Current kernel in first order. The current kernel $\Sigma_{\gamma}^{(1)}(E, \omega)$ can be determined similarly to $L_{S}^{(1)}(E, \omega)$, one just has to replace the first vertex on the r.h.s. of the RG equations (130) and (131) by the current vertex (251). Using the identities (253) and (254) together with the algebra of the basis superoperators, we obtain zero on the r.h.s. of the $\mathrm{RG}$ equations, i.e.,

$$
\Sigma_{\gamma}^{(1)}(E, \omega)=0
$$

Vertices in second order. The vertices in second order in $J$ follow from the decomposition (144), where the first (imaginary) part $i \bar{G}_{11^{\prime}}^{\left(2 a_{1}\right)}$ (or $i \bar{I}_{11^{\prime}}^{\gamma\left(2 a_{1}\right)}$ for the current kernel) is given by Eq. (145). Since the renormalized vertices in first order have the same form as the initial ones, we obtain the same form as Eqs. (261) and (262) with $\hat{J}_{0}^{z / \perp} \rightarrow \hat{J}^{z / \perp}$

$$
\begin{aligned}
\bar{G}_{11^{\prime}}^{\left(2 a_{1}\right)}= & \frac{\pi}{2} \hat{J}^{\perp} \hat{J}^{\perp} s \hat{L}_{s}^{3 z} \\
& +\frac{\pi}{4}\left(\hat{J}^{z} \hat{J}^{\perp}+\hat{J}^{\perp} \hat{J}^{z}\right) \hat{L}_{s}^{3}, \\
\bar{I}_{11^{\prime}}^{\gamma\left(2 a_{1}\right)}= & -\frac{\pi}{8}\left(\hat{J}^{\gamma z} \hat{J}^{z}-\hat{J}^{z} \hat{J}^{\gamma z}\right. \\
& \left.+2 \hat{J}^{\gamma \perp} \hat{J}^{\perp}-2 \hat{J}^{\perp} \hat{J}^{\gamma \perp}\right) \hat{L}_{s}^{b} .
\end{aligned}
$$

In contrast, the (real) parts $\bar{G}_{11^{\prime}}^{\left(2 a_{2}\right)}$ and $\bar{I}_{11^{\prime}}^{\gamma\left(2 a_{2}\right)}$ have to be calculated from the RG equation (143) with zero initial condition. We use the same ansatz (263) and (264) as for the vertices in lowest order, i.e., for $\eta=-\eta^{\prime}=+$

$$
\begin{aligned}
\bar{G}_{11^{\prime}}^{\left(2 a_{2}\right)} & =\frac{1}{2} \hat{K}^{z} s \hat{L}_{s}^{h}-\frac{1}{2} \hat{K}^{\perp}\left(\hat{L}_{s}^{4}+\hat{L}_{s}^{5}\right) \\
\bar{I}_{11^{\prime}}^{\gamma\left(2 a_{2}\right)} & =\frac{1}{2} \hat{K}^{\gamma z} s \hat{L}_{s}^{1 z}+\frac{1}{2} \hat{K}^{\gamma \perp} \hat{L}_{s}^{1}
\end{aligned}
$$

with $\hat{K}^{z / \perp}, \hat{K}^{\gamma, z / \perp} \sim O\left(J^{2}\right)$. However, instead of Eq. (265), we set

$$
K_{\alpha \alpha^{\prime}}^{\gamma, z / \perp}=c_{\alpha \alpha^{\prime}}^{\gamma} K_{\alpha \alpha^{\prime}}^{z / \perp}+R_{\alpha \alpha^{\prime}}^{\gamma, z / \perp}
$$

which can be viewed as a definition of $\hat{R}^{\gamma, z / \perp}$. Inserting Eqs. (285), (286), (263), (264), and (280) into the RG equation (143), and using the following properties [analog 
to (260)], valid for $\eta=-\eta^{\prime}=+$ :

$$
\begin{gathered}
A_{12} L^{\chi} B_{\overline{2} 1^{\prime}}-\left(1 \leftrightarrow 1^{\prime}\right)=A^{\chi^{\prime} s^{\prime}} B^{\chi^{\prime \prime} s^{\prime \prime}} \hat{L}_{s^{\prime}}^{\chi^{\prime}} L^{\chi} \hat{L}_{s^{\prime \prime}}^{\chi^{\prime \prime}}- \\
-B^{\chi^{\prime \prime} s^{\prime \prime}} A^{\chi^{\prime} s^{\prime}}\left(\left(\hat{L}_{s^{\prime}}^{\chi^{\prime}}\right)^{T} L^{\chi}\left(\hat{L}_{s^{\prime \prime}}^{\chi^{\prime \prime}}\right)^{T}\right)^{T}, \\
A_{23} B_{11^{\prime}} C_{\overline{3} \overline{2}}=2 B_{\alpha \alpha^{\prime}}^{\chi s}\left(\operatorname{Tr}_{\alpha} A^{\chi^{\prime} s^{\prime}} C^{\chi^{\prime \prime} s^{\prime \prime}}\right) \\
\cdot\left(\operatorname{Tr}_{\sigma} \hat{L}_{s^{\prime}}^{\chi^{\prime}}\left(\hat{L}_{s}^{\chi}\right)_{\sigma \sigma^{\prime}} \hat{L}_{s^{\prime \prime}}^{\chi^{\prime \prime}}\right)
\end{gathered}
$$

we obtain after some lengthy but straighforward algebra the following $\mathrm{RG}$ equations:

$$
\begin{aligned}
& \frac{d}{d l} \hat{K}^{z}=-\left(\operatorname{Tr}\left(\hat{J}^{z}-\hat{J}_{0}^{z}\right)\right) \hat{J}^{\perp} \hat{J}^{\perp}+\frac{1}{2}\left(\operatorname{Tr} \hat{J}^{z} \hat{J}^{z}\right) \hat{J}^{z}+\hat{K}^{\perp} \hat{J}^{\perp}+\hat{J}^{\perp} \hat{K}^{\perp} \\
& \frac{d}{d l} \hat{K}^{\perp}=-\left(\operatorname{Tr}\left(\hat{J}^{z}-\hat{J}_{0}^{z}\right)+2 c^{2} l\right) \frac{1}{2}\left(\hat{J}^{z} \hat{J}^{\perp}+\hat{J}^{\perp} \hat{J}^{z}\right)+\frac{1}{2}\left(\operatorname{Tr} \hat{J}^{\perp} \hat{J}^{\perp}\right) \hat{J}^{\perp} \\
& +\frac{1}{2}\left(\hat{K}^{\perp} \hat{J}^{z}+\hat{J}^{z} \hat{K}^{\perp}+\hat{K}^{z} \hat{J}^{\perp}+\hat{J}^{\perp} \hat{K}^{z}\right) \\
& \frac{d}{d l} \hat{R}^{\gamma z}=-\frac{1}{2}\left(\operatorname{Tr} \hat{J}^{z} \hat{J}^{z}\right) \hat{J}^{\gamma z}-2 c^{2} l\left(\hat{J}^{\gamma \perp} \hat{J}^{\perp}+\hat{J}^{\perp} \hat{J}^{\gamma \perp}\right)+\hat{R}^{\gamma \perp} \hat{J}^{\perp}+\hat{J}^{\perp} \hat{R}^{\gamma \perp} \\
& \frac{d}{d l} \hat{R}^{\gamma \perp}=-\frac{1}{2}\left(\operatorname{Tr} \hat{J}^{\perp} \hat{J}^{\perp}\right) \hat{J}^{\gamma \perp}+c^{2} l\left(\hat{J}^{\gamma z} \hat{J}^{\perp}+\hat{J}^{\perp} \hat{J}^{\gamma z}\right)+\frac{1}{2}\left(\hat{R}^{\gamma \perp} \hat{J}^{z}+\hat{J}^{z} \hat{R}^{\gamma \perp}+\hat{R}^{\gamma z} \hat{J}^{\perp}+\hat{J}^{\perp} \hat{R}^{\gamma z}\right) .
\end{aligned}
$$

Together with Eq. (287), we have derived here the full two-loop equations for the vertex and the current vertex in the most general case, including anisotropies and all possibilities for the dependencies on the reservoir indices. If we define the total vertex by

$$
\hat{\mathcal{J}}^{z / \perp}=\hat{J}^{z / \perp}+\hat{K}^{z / \perp}
$$

and take the sum of the RG equations (266), (290), and (291), we get, by neglecting terms of $O\left(J^{4}\right)$ and terms of $O\left(J_{0} J^{2}, J_{0}^{2} J\right)$ (which vanish in the scaling limit $\left.J_{0} \rightarrow 0\right)$, the following result:

$$
\begin{aligned}
\frac{d}{d l} \hat{\mathcal{J}}^{z} & =\left(1-\operatorname{Tr} \hat{\mathcal{J}}^{z}\right)\left(\hat{\mathcal{J}}^{\perp}\right)^{2}+\frac{1}{2}\left[\operatorname{Tr}\left(\hat{\mathcal{J}}^{z}\right)^{2}\right] \hat{\mathcal{J}}^{z} \\
\frac{d}{d l} \hat{\mathcal{J}}^{\perp} & =\left(1-\operatorname{Tr} \hat{\mathcal{J}}^{z}\right) \frac{1}{2}\left(\hat{\mathcal{J}}^{z} \hat{\mathcal{J}}^{\perp}+\hat{\mathcal{J}}^{\perp} \hat{\mathcal{J}}^{z}\right)+\frac{1}{2}\left[\operatorname{Tr}\left(\hat{\mathcal{J}}^{\perp}\right)^{2}\right] \hat{\mathcal{J}}^{\perp} .
\end{aligned}
$$

For $\hat{\mathcal{J}}^{z}=\hat{\mathcal{J}}^{\perp}=\hat{\mathcal{J}}$ and the case of two reservoirs with and $\mathcal{J}_{L L}=\mathcal{J}_{R R}$, these equations reduce to the two-loop equations of Ref. 35 [where the nonuniversal parameter $a$ in Eq. (104) of this reference has to be chosen as $a=3]$. In equilibrium (i.e., for a single reservoir), we recover the well-known two-loop RG equations for the anisotropic Kondo model derived in Ref. 36 [up to nonuniversal terms arising from adding terms proportional to the invariant $\left.\operatorname{Tr}\left(\hat{J}^{z} \hat{J}^{z}-\hat{J}^{\perp} \hat{J}^{\perp}\right)\right]$.

If the exchange couplings fulfil the relation (203), the $\mathrm{RG}$ equations can be solved easily. In this case, we have

$$
R_{\alpha \alpha^{\prime}}^{\gamma, z / \perp}=c_{\alpha \alpha^{\prime}}^{\gamma} R_{\alpha \alpha^{\prime}}^{z / \perp}
$$

$$
\begin{aligned}
K_{\alpha \alpha^{\prime}}^{z / \perp} & =2 \sqrt{x_{\alpha} x_{\alpha^{\prime}}} K^{z / \perp}, \\
R_{\alpha \alpha^{\prime}}^{z / \perp} & =2 \sqrt{x_{\alpha} x_{\alpha^{\prime}}} R^{z / \perp},
\end{aligned}
$$


with

$$
\begin{aligned}
\frac{d}{d l} K^{z}= & -4\left(J^{z}-J_{0}^{z}\right)\left(J^{\perp}\right)^{2} \\
& +2\left(J^{z}\right)^{3}+4 J^{\perp} K^{\perp} \\
\frac{d}{d l} K^{\perp}= & -4\left(J^{z}-J_{0}^{z}+2 c^{2} l\right) J^{z} J^{\perp} \\
& +2\left(J^{\perp}\right)^{3}+2\left(J^{z} K^{\perp}+J^{\perp} K^{z}\right) \\
\frac{d}{d l} R^{z}= & -2\left(J^{z}\right)^{3}-4 c^{2} l\left(J^{\perp}\right)^{2}+2 J^{\perp} R^{\perp} \\
\frac{d}{d l} R^{\perp}= & -2\left(J^{\perp}\right)^{3}+2 c^{2} l J^{z} J^{\perp} \\
& +J^{z} R^{\perp}+J^{\perp} R^{z} .
\end{aligned}
$$

Special solutions of these equations are given by

$$
\begin{aligned}
K^{z}= & \frac{3}{2} c^{2}+2 l c^{2} J^{z} \\
& +4 l J_{0}^{z}\left(J^{\perp}\right)^{2}-\left(J^{\perp}\right)^{2} \ln \frac{J^{\perp}}{J_{0}^{\perp}} \\
K^{\perp}= & l c^{2} J^{\perp}+4 l J_{0}^{z} J^{z} J^{\perp}-J^{z} J^{\perp} \ln \frac{J^{\perp}}{J_{0}^{\perp}}, \\
R^{z}= & -\left(J^{z}\right)^{2}-2 c^{2} l J^{z}, R^{\perp}=-J^{z} J^{\perp}
\end{aligned}
$$

and the initial conditions $K^{z}=K^{\perp}=R^{z}=R^{\perp}=0$ can be fulfilled by adding linear combinations of the following solutions of the homogeneous part of the RG equations:

$$
\begin{aligned}
K^{z}=\left(J^{\perp}\right)^{2} & , K^{\perp}=J^{z} J^{\perp} \\
K^{z}=J^{z}+2 l\left(J^{\perp}\right)^{2} & , K^{\perp}=J^{\perp}+2 l J^{z} J^{\perp} \\
R^{z}=J^{z} & , R^{\perp}=J^{\perp} .
\end{aligned}
$$

As a consequence, the final solutions are given by

$$
\begin{aligned}
K^{z}= & \frac{3}{2} J^{z}\left(J^{z}-J_{0}^{z}\right)+2 l c^{2} J^{z} \\
& +l J_{0}^{z}\left(J^{\perp}\right)^{2}-\left(J^{\perp}\right)^{2} \ln \frac{J^{\perp}}{J_{0}^{\perp}}, \\
K^{\perp}= & \frac{3}{2} J^{\perp}\left(J^{z}-J_{0}^{z}\right)+l c^{2} J^{\perp} \\
& +l J_{0}^{z} J^{z} J^{\perp}-J^{z} J^{\perp} \ln \frac{J^{\perp}}{J_{0}^{\perp}}, \\
R^{z}= & J^{z}\left(J_{0}^{z}-J^{z}\right)-2 c^{2} l J^{z}, \\
R^{\perp}= & J^{\perp}\left(J_{0}^{z}-J^{z}\right) .
\end{aligned}
$$

Using Eq. 281) and $c^{2}=\left(J^{z}\right)^{2}-\left(J^{\perp}\right)^{2}$, we see that the vertices in second order contain four different types of terms

$$
O\left(J_{0} J\right), O\left(J^{2}\right), O\left(J^{2} \ln J\right), O\left(J^{2} \ln J_{0}\right)
$$

in addition to factors which contain ratios of $J^{z} / J^{\perp}$ or $J_{0}^{z} / J_{0}^{\perp}$. In the scaling limit, the terms of order $O\left(J_{0} J\right)$ can be neglected. The terms of order $O\left(J^{2}\right)$ are just a perturbative correction to the first-order vertices and can be neglected too. Therefore, we see that the terms $R^{z / \perp}$ are not important and the current vertex (287) in second order can be written as

$$
K_{\alpha \alpha^{\prime}}^{\gamma, z / \perp} \approx c_{\alpha \alpha^{\prime}}^{\gamma} K_{\alpha \alpha^{\prime}}^{z / \perp}
$$

The terms of order $O\left(J^{2} \ln J\right)$ are logarithmic corrections, which are of order $J \ln J$ smaller compared to the firstorder vertices. Since $J \ll 1$ they are also perturbative corrections and can be omitted. They lead only to an overall change of the physical quantities without any interesting dependence on some physical energy scale (like e.g. the voltage, magnetic field, etc.). The most important terms are those of order $O\left(J^{2} \ln J_{0}\right)$ since they diverge in the scaling limit. Therefore, they have to be incorporated into the definition of the Kondo temperature. Using the solution (274) with $T_{K} \rightarrow T_{K}^{\prime}$ and expanding in $J \ln \frac{T_{K}^{\prime}}{T_{K}}$, we find

$$
\begin{aligned}
& \left(J^{z}\right)^{\prime}=c \frac{1+\left(\frac{T_{K}^{\prime}}{\Lambda}\right)^{4 c}}{1-\left(\frac{T_{K}^{\prime}}{\Lambda}\right)^{4 c}} \approx J^{z}+2\left(J^{\perp}\right)^{2} \ln \frac{T_{K}^{\prime}}{T_{K}}, \\
& \left(J^{\perp}\right)^{\prime}=2 c \frac{\left(\frac{T_{K}^{\prime}}{\Lambda}\right)^{2 c}}{1-\left(\frac{T_{K}^{\prime}}{\Lambda}\right)^{4 c}} \approx J^{\perp}+2 J^{z} J^{\perp} \ln \frac{T_{K}^{\prime}}{T_{K}},
\end{aligned}
$$

i.e., the terms of order $O\left(J^{2} \ln J_{0}^{\perp}\right)$ in Eqs. (310) and (311) can be acccounted for by the redefinition

$$
T_{K}^{\prime}=\sqrt{J_{0}^{\perp}} T_{K}
$$

As a consequence, the main effect of the two-loop terms for the vertices is the replacement $T_{K} \rightarrow T_{K}^{\prime}$, which we will always implicitly assume in the following.

In other (more academic) cases, where the form (203) of the exchange couplings is not fulfilled, the part $R_{\alpha \alpha^{\prime}}^{\gamma z / \perp}$ can generally not be written in the form (297), i.e., $\gamma$ must not necessarily be equal to $\alpha$ or $\alpha^{\prime}$. From the RG equations (292) and (293), one can only prove that

$$
R_{\alpha \alpha^{\prime}}^{\gamma z / \perp}=-R_{\alpha^{\prime} \alpha}^{\gamma z / \perp} \quad, \quad \sum_{\gamma} R_{\alpha \alpha^{\prime}}^{\gamma z / \perp}=0 .
$$

However, in the special case of two reservoirs, these conditions lead again to the form (297). It is an open question of future research to analyze the two-loop equations in all cases and to find out whether there are interesting situations where the two-loop contributions can not be simply accounted for by a renormalization of a single parameter. In addition, it is not clear whether the corrections $R_{\alpha \alpha^{\prime}}^{\gamma, z / \perp}$ to the current vertex are generically unimportant. In this paper, we discuss only the physically realizable situation, where the exchange couplings fulfil the property (203).

Liouvillian in second order. For Eq. (193), we need the following second-order contribution to the Liouvillian:

$$
L_{S}^{(2 b)}(E, \omega)=L_{S}^{(2 b)}-(E+i \omega) Z^{(2 b)}
$$


where $L_{S}^{(2 b)}$ and $Z^{(2 b)}$ are determined by the RG equations (158) and (159) with initial values given by Eqs. (257) and (259). Inserting the results (263) and (283) for the vertices into Eqs. (158) and (159), and using the identities (253) and (254), we find after some algebra the $\mathrm{RG}$ equations

$$
\begin{aligned}
& \frac{d}{d l} L_{S}^{(2 b)}=-i \pi h_{0}\left(\operatorname{Tr} \hat{J}^{\perp} \hat{J}^{\perp} \hat{J}^{z}\right) L^{3 z}, \\
& \frac{d}{d l} Z^{(2 b)}=0
\end{aligned}
$$

Using $\left(\hat{J}^{\perp}\right)^{2}=\frac{d}{d l} \hat{J}^{z}$ according to Eq. (266) together with the inital condition (257), we find the result

$$
\begin{aligned}
& L_{S}^{(2 b)}=-i \frac{\pi}{2} h_{0}\left(\operatorname{Tr} \hat{J}^{\perp} \hat{J}^{\perp}\right) L^{3 z}, \\
& Z_{S}^{(2 b)}=0,
\end{aligned}
$$

where we have used that

$$
4 c^{2}=\operatorname{Tr}\left(\hat{J}^{z} \hat{J}^{z}-\hat{J}^{\perp} \hat{J}^{\perp}\right)=\operatorname{Tr}\left(\hat{J}_{0}^{z} \hat{J}_{0}^{z}-\hat{J}_{0}^{\perp} \hat{J}_{0}^{\perp}\right)
$$

is an invariant according to Eq. (267).

Current kernel in second order. Analog to $L_{S}^{(2 b)}(E, \omega)$, we evaluate the current kernel

$$
\Sigma_{\gamma}^{(2 b)}(E, \omega)=\Sigma_{\gamma}^{(2 b)}-(E+i \omega) Z_{\gamma}^{(2 b)}
$$

from the RG equations (158) and (159) by using the results (264) and (284) for the current vertices. This gives the $\mathrm{RG}$ equations

$$
\begin{aligned}
\frac{d}{d l} \Sigma_{\gamma}^{(2 b)}=-i & \frac{\pi}{2} c_{\alpha \alpha^{\prime}}^{\gamma}\left(\mu_{\alpha}-\mu_{\alpha^{\prime}}\right)\left\{J_{\alpha \alpha^{\prime}}^{z}\left(\hat{J}^{\perp} \hat{J}^{\perp}\right)_{\alpha^{\prime} \alpha}+\right. \\
& \left.+J_{\alpha \alpha^{\prime}}^{\perp}\left(\hat{J}^{z} \hat{J}^{\perp}+\hat{J}^{\perp} \hat{J}^{z}\right)_{\alpha^{\prime} \alpha}\right\} L^{b},(324)
\end{aligned}
$$

and $\frac{d}{d l} Z_{\gamma}^{(2 b)}=0$. Using $\hat{J}^{\perp} \hat{J}^{\perp}=\frac{d}{d l} \hat{J}^{z}$ and $\hat{J}^{z} \hat{J}^{\perp}+$ $\hat{J}^{\perp} \hat{J}^{z}=2 \frac{d}{d l} \hat{J}^{\perp}$ according to Eq. (266), we find together with the initial conditions (258) and (259) the result

$$
\begin{aligned}
& \Sigma_{\gamma}^{(2 b)}=-i \frac{\pi}{4} c_{\alpha \alpha^{\prime}}^{\gamma}\left(\mu_{\alpha}-\mu_{\alpha^{\prime}}\right)\left[\left(J_{\alpha \alpha^{\prime}}^{z}\right)^{2}+2\left(J_{\alpha \alpha^{\prime}}^{\perp}\right)^{2}\right] L^{b}, \\
& Z_{\gamma}^{(2 b)}=0 .
\end{aligned}
$$

\section{2. $R G$ below $\Lambda_{c}$}

We now start to evaluate the final formulas (192)-(195) for the effective Liouvillian and the current kernel, which are represented in terms of the basis superoperators according to the forms (222) and (223). All final quantities are evaluated at $\omega=0$ and are meant at $\Lambda=0$ which is not indicated explicitly, i.e., we write from now on

$$
\begin{aligned}
L_{S}(E) \equiv & L_{S}(E, \omega=0)_{\Lambda=0}= \\
= & h(E) L^{h}-i \Gamma^{a}(E) L^{a} \\
& -i \Gamma^{c}(E) L^{c}-i \Gamma^{3 z}(E) L^{3 z}, \\
\Sigma_{\gamma}(E) \equiv & \Sigma_{\gamma}(E, \omega=0)_{\Lambda=0}= \\
= & i \Gamma_{\gamma}^{b}(E) L^{b}+i \Gamma_{\gamma}^{1 z}(E) L^{1 z} .
\end{aligned}
$$

Since only the first-order vertices evaluated at $\Lambda=\Lambda_{c}$ occur in the final formulas, we will furthermore use the convention

$$
\left.J_{\alpha \alpha^{\prime}}^{z} \equiv\left(J_{\alpha \alpha^{\prime}}^{z}\right)\right|_{\Lambda=\Lambda_{c}} \quad,\left.\quad J_{\alpha \alpha^{\prime}}^{\perp} \equiv\left(J_{\alpha \alpha^{\prime}}^{\perp}\right)\right|_{\Lambda=\Lambda_{c}} .
$$

According to Eq. (111), the scale $\Lambda_{c}$ is defined as the maximum of all physical energy scales, i.e., in our case of the Kondo problem

$$
\Lambda_{c}=\max \{E, V, \tilde{h}\},
$$

where $\tilde{h}$ is defined by the renormalized magnetic field or, more precisely, as the real part of the eigenvalues of the operator $\tilde{L}_{S}$. Therefore, we have to keep in mind that the renormalized exchange couplings (328) depend implicitly on the variables $E, V$, and $\tilde{h}$ via the scale $\Lambda_{c}$.

According to Eq. (55), any function $\mathcal{H}\left(\Delta_{12}\right)$ of the quantities $\Delta_{12}=E_{12}-\tilde{L}_{S}$ is defined by

$$
\mathcal{H}\left(\Delta_{12}\right)=\sum_{i} \mathcal{H}\left(E_{12}-z_{i}\right) P_{i}\left(z_{i}\right),
$$

with $i \equiv 0,1, \pm$ and $z_{i}=\lambda_{i}\left(z_{i}\right) . \quad \lambda_{i}(z) \equiv \lambda(E, \omega)$ and $P_{i}(z) \equiv P_{i}(E, \omega)$ are given by Eqs. (227) and (228), with $z \equiv E+i \omega$. As a consequence, we obtain the following self-consistent equations for $z_{i}$ :

$$
\begin{aligned}
z_{0} & =0 \\
z_{1} & =-i \Gamma^{a}\left(z_{1}\right), \\
z_{ \pm} & = \pm h\left(z_{ \pm}\right)-i\left(\Gamma^{a}+\Gamma^{c}\right)\left(z_{ \pm}\right) .
\end{aligned}
$$

The pole $z_{0}=0$ corresponds to the stationary state, which does not occur in the resolvents between the renormalized vertices due to our special construction, where the eigenvalue zero is perturbatively integrated out during the first discrete RG step. The poles $z_{1}$ and $z_{ \pm}$correspond to the spin relaxation and dephasing modes. Using the symmetry properties (224) and (226), we obtain under complex conjugation

$$
\begin{aligned}
z_{1}^{*} & =i \Gamma^{a}\left(-z_{1}^{*}\right), \\
z_{ \pm}^{*} & = \pm h\left(-z_{ \pm}^{*}\right)+i\left(\Gamma^{a}+\Gamma^{c}\right)\left(-z_{ \pm}^{*}\right),
\end{aligned}
$$

which gives

$$
\begin{aligned}
z_{1} & =-z_{1}^{*} \equiv-i \tilde{\Gamma}_{1} \quad \\
z_{ \pm} & =-z_{\mp}^{*}= \pm \tilde{h}-i \tilde{\Gamma}_{2} \quad .
\end{aligned}
$$

As a consequence, Eq. (330) becomes

$$
\begin{aligned}
& \mathcal{H}\left(\Delta_{12}\right)=\mathcal{H}\left(E_{12}\right) P_{0}(0)+\mathcal{H}\left(E_{12}+i \tilde{\Gamma}_{1}\right) P_{1}\left(-i \tilde{\Gamma}_{1}\right) \\
& \quad+\mathcal{H}\left(E_{12}-s \tilde{h}+i \tilde{\Gamma}_{2}\right) P_{s}\left(s \tilde{h}-i \tilde{\Gamma}_{2}\right) .
\end{aligned}
$$

where we sum over $s= \pm$.

Inserting Eq. (338) for the various functions occurring in Eq. (193) - 195), we see that the projectors stand always left to $\bar{G}_{i j}^{(1) c}$ or $\bar{G}_{i j}^{\left(2 a_{1}\right) c}$. Using the results (263) and (283) for these vertices together with the algebra of the 
basis superoperators, we conclude that the parts of the projectors (228) containing either $L^{b}$ or $L^{3 z}$ do not contribute, and we can replace $L^{a} \rightarrow 1$. Thus, by inserting Eq. (228) in Eq. (338), we obtain the useful identity

$$
\begin{aligned}
& \mathcal{H}\left(\Delta_{12}\right) \rightarrow \mathcal{H}\left(E_{12}+i \tilde{\Gamma}_{1}\right)\left(1-L^{c}\right) \\
& \quad+\mathcal{H}\left(E_{12}-s \tilde{h}+i \tilde{\Gamma}_{2}\right) \frac{1}{2}\left(L^{c}+s L^{h}\right),
\end{aligned}
$$

which will be used frequently during the following analysis. As a consequence, we find that the spin relaxation rate $\tilde{\Gamma}_{1}$ will cut off all logarithms where the magnetic field does not occur, and the spin dephasing rates $\tilde{\Gamma}_{2}$ cuts off all logarithms where the magnetic field occurs. This is expected since the spin dephasing rate corresponds to spin flip processes. In addition, we also conclude from Eq. (339) that the pole of the projector $P_{1}(E, \omega)$ at $\Gamma^{a}(E, \omega)=0$ does not contribute.

Liouvillian and current kernel in first order. The Liouvillian and the current kernel in first order in $J$ have already been determined in Eqs. (278)-(280) and (282), which gives

$$
\begin{aligned}
h^{(1)}(E) & =\frac{1}{2} \operatorname{Tr}\left(\hat{J}^{z}-\hat{J}_{0}^{z}\right) h_{0}+2 c^{2} l_{c} h_{0}, \\
\Gamma^{a(1)}(E) & =-i E \operatorname{Tr}\left(\hat{J}^{z}-\hat{J}_{0}^{z}\right) \\
\Gamma^{c(1)}(E) & =-2 i E c^{2} l_{c} \\
\Gamma^{3 z(1)}(E) & =0
\end{aligned}
$$

with $l_{c}=\ln \frac{\Lambda_{0}}{\Lambda_{c}}$, and

$$
\Gamma_{\gamma}^{b(1)}(E)=\Gamma_{\gamma}^{1 z(1)}(E)=0
$$

Liouvillian and current kernel in second order. The first two terms on the r.h.s. of Eq. (193) have already been evaluated in Eqs. (321), (322), and (325). For the evaluation of the last two terms on the r.h.s., we use Eq. (339) and the identity $[\mathcal{H}(E)$ is any function]

$$
\begin{aligned}
& \mathcal{H}\left(E_{11^{\prime}}+z\right) A_{11^{\prime}} L^{\chi} B_{\overline{1}^{\prime} \overline{1}}= \\
& \quad=2 \mathcal{H}\left(E_{\alpha \alpha^{\prime}}+z\right) A_{\alpha \alpha^{\prime}}^{\chi^{\prime} s^{\prime}} B_{\alpha^{\prime} \alpha}^{\chi^{\prime \prime} s^{\prime \prime}} \operatorname{Tr}_{\sigma} \hat{L}_{s^{\prime}}^{\chi^{\prime}} L^{\chi} \hat{L}_{s^{\prime \prime}}^{\chi^{\prime \prime}},
\end{aligned}
$$

together with the results (263) and (264) for the firstorder vertices. After some algebra, we obtain for the components of the effective Liouvillian in second order

$$
\begin{gathered}
\operatorname{Re} h^{(2)}(E)=-\frac{1}{4}\left(E_{\alpha \alpha^{\prime}}-\tilde{h}\right) \mathcal{L}_{2}\left(E_{\alpha \alpha^{\prime}}-\tilde{h}\right)\left(J_{\alpha \alpha^{\prime}}^{z}\right)^{2} \\
+(E \rightarrow-E), \\
\operatorname{Im} h^{(2)}(E)=-\frac{\pi}{8}\left|E_{\alpha \alpha^{\prime}}-\tilde{h}\right|\left(J_{\alpha \alpha^{\prime}}^{z}\right)^{2}-(E \rightarrow-E), \\
\operatorname{Re} \Gamma^{a(2)}(E)=\frac{\pi}{4}\left|E_{\alpha \alpha^{\prime}}-\tilde{h}\right|\left(J_{\alpha \alpha^{\prime}}^{\perp}\right)^{2}+(E \rightarrow-E), \\
\operatorname{Im} \Gamma^{a(2)}(E)=-\frac{1}{2}\left(E_{\alpha \alpha^{\prime}}-\tilde{h}\right) \mathcal{L}_{2}\left(E_{\alpha \alpha^{\prime}}-\tilde{h}\right)\left(J_{\alpha \alpha^{\prime}}^{\perp}\right)^{2} \\
\quad-(E \rightarrow-E) \quad, \\
\operatorname{Re}\left[\Gamma^{a(2)}(E)+\Gamma^{c(2)}(E)\right]= \\
=\frac{\pi}{8}\left\{\left|E_{\alpha \alpha^{\prime}}-\tilde{h}\right|\left(J_{\alpha \alpha^{\prime}}^{z}\right)^{2}+\left|E_{\alpha \alpha^{\prime}}\right|\left(J_{\alpha \alpha^{\prime}}^{\perp}\right)^{2}\right\} \\
\quad+(E \rightarrow-E) \quad, \\
\operatorname{Im}\left[\Gamma^{a(2)}(E)+\Gamma^{c(2)}(E)\right]= \\
=-\frac{1}{4}\left\{\left(E_{\alpha \alpha^{\prime}}-\tilde{h}\right) \mathcal{L}_{2}\left(E_{\alpha \alpha^{\prime}}-\tilde{h}\right)\left(J_{\alpha \alpha^{\prime}}^{z}\right)^{2}+\right. \\
\left.+E_{\alpha \alpha^{\prime}} \mathcal{L}_{1}\left(E_{\alpha \alpha^{\prime}}\right)\left(J_{\alpha \alpha^{\prime}}^{\perp}\right)^{2}\right\}-(E \rightarrow-E), \\
\Gamma^{3 z(2)}(E)=\frac{\pi}{2} h_{0} \operatorname{Tr} \hat{J}^{\perp} \hat{J}^{\perp} \quad,
\end{gathered}
$$

and the following result for the components of the current kernel in second order:

$$
\begin{gathered}
\Gamma_{\gamma}^{b(2)}(E)=-\frac{\pi}{4}\left(\mu_{\alpha}-\mu_{\alpha^{\prime}}\right)\left(J_{\alpha \alpha^{\prime}}^{\gamma z} J_{\alpha \alpha^{\prime}}^{z}+2 J_{\alpha \alpha^{\prime}}^{\gamma \perp} J_{\alpha \alpha^{\prime}}^{\perp}\right), \\
\operatorname{Re}_{\gamma}^{1 z(2)}(E)=\frac{\pi}{4}\left|E_{\alpha \alpha^{\prime}}-\tilde{h}\right| J_{\alpha \alpha^{\prime}}^{\gamma \perp} J_{\alpha \alpha^{\prime}}^{\perp}+(E \rightarrow-E), \\
\operatorname{Im} \Gamma_{\gamma}^{1 z(2)}(E)=-\frac{1}{2}\left|E_{\alpha \alpha^{\prime}}-\tilde{h}\right| \mathcal{L}_{2}\left(E_{\alpha \alpha^{\prime}}-\tilde{h}\right) J_{\alpha \alpha^{\prime}}^{\gamma \perp} J_{\alpha \alpha^{\prime}}^{\perp} \\
-(E \rightarrow-E),
\end{gathered}
$$

where we have used the shorthand notation

$$
\mathcal{L}_{i}(x):=\ln \frac{\Lambda_{c}}{\sqrt{x^{2}+\left(\tilde{\Gamma}_{i}\right)^{2}}}
$$

for the logarithmic terms. Note that the results respect the symmetry properties (224)-(226), i.e., the real parts of all quantities are symmetric in the Laplace variable $E$, whereas the imaginary parts are antisymmetric in $E$.

As we can see, for finite Laplace variable $E$, logarithmic corrections occur already in second order in $J$. This is the case for the real part of $h^{(2)}(E)$ and the imaginary parts of $\Gamma^{a(2)}(E), \Gamma^{c(2)}(E)$, and $\Gamma_{\gamma}^{1 z(2)}(E)$. Therefore, in the stationary state (i.e., for $E=0$ ) no logarithmic contributions occur in $O\left(J^{2}\right)$ for the conductance and the magnetization, whereas the time evolution is influenced by logarithmic contributions already in this order, see Sec. IVA for more details.

Liouvillian and current kernel in third order. In third order, there are two contributions to the effective Liouvillian and the current kernel, denoted by the superscript 
(3a) and (3b) and given by the two expressions, Eqs. (194) and (195). The components $(3 a)$ from Eq. (194) can be evaluated similiar to the components in second order from Eq. (193) shown above, just by using in addition the results (283) and (284) for the imaginary part of the vertices in second order. We obtain after some algebra for the components of the effective Liouvillian

$$
\begin{gathered}
h^{(3 a)}(E)=\Gamma^{a(3 a)}(E)=\Gamma^{c(3 a)}(E)=0, \\
\Gamma^{3 z(3 a)}(E)=-\frac{\pi}{4}\left(E_{\alpha \alpha^{\prime}}-\tilde{h}\right) \mathcal{L}_{2}\left(E_{\alpha \alpha^{\prime}}-\tilde{h}\right) . \\
\cdot J_{\alpha \alpha^{\prime}}^{\perp}\left(\hat{J}^{z} \hat{J}^{\perp}+\hat{J}^{\perp} \hat{J}^{z}\right)_{\alpha^{\prime} \alpha}+(E \rightarrow-E),
\end{gathered}
$$

and for the components for the current kernel

$$
\begin{gathered}
\Gamma_{\gamma}^{b(3 a)}(E)=-\frac{\pi}{4}\left\{E_{\alpha \alpha^{\prime}} \mathcal{L}_{1}\left(E_{\alpha \alpha^{\prime}}\right) J_{\alpha \alpha^{\prime}}^{\gamma z}\left(\hat{J}^{\perp} \hat{J}^{\perp}\right)_{\alpha^{\prime} \alpha}+\right. \\
\left.+\left(E_{\alpha \alpha^{\prime}}-\tilde{h}\right) \mathcal{L}_{2}\left(E_{\alpha \alpha^{\prime}}-\tilde{h}\right) J_{\alpha \alpha^{\prime}}^{\gamma \perp}\left(\hat{J}^{z} \hat{J}^{\perp}+\hat{J}^{\perp} \hat{J}^{z}\right)_{\alpha^{\prime} \alpha}\right\} \\
+(E \rightarrow-E) .
\end{gathered}
$$

As expected, the logarithmic terms for the real parts of $\Gamma^{3 z}$ and $\Gamma_{\gamma}^{b}$ start in order $J^{3}$. This means that the logarithmic terms for the stationary magnetization (230) and the stationary current (231) start in third order in the exchange couplings. Note that we have not calculated the terms in order $O\left(J^{3}\right)$ without a logarithmic contribution.

The remaining logarithmic contributions in third order are contained in the component (3b), given by (195) and
(197). With $A \equiv \bar{G}^{(1) c}$ and $B \equiv \bar{I}^{\gamma(1) c}$ we have to evaluate expressions of the form [note that $\left(A^{\chi^{s}}\right)^{T}=A$ and $\left(B^{\chi s}\right)^{T}=-B$ according to Eqs. (263), (264), and (202)]

$$
\begin{aligned}
& (A / B)_{12} R\left(\Delta_{12}\right) A_{\overline{2} 3} S\left(\Delta_{13}\right) A_{\overline{3} \overline{1}}= \\
& =(A / B)_{\alpha_{1} \alpha_{2}}^{\chi s} A_{\alpha_{2} \alpha_{3}}^{\chi^{\prime} s^{\prime}} A_{\alpha_{3} \alpha_{1}}^{\chi^{\prime \prime} s^{\prime \prime}} . \\
& \quad \operatorname{Tr}_{\sigma}\left\{\hat{L}_{s}^{\chi} R\left(\Delta_{\alpha_{1} \alpha_{2}}\right) \hat{L}_{s^{\prime}}^{\chi^{\prime}} S\left(\Delta_{\alpha_{1} \alpha_{3}}\right) \hat{L}_{s^{\prime \prime}}^{\chi^{\prime \prime}-}\right. \\
& \left.\quad-\left(\hat{L}_{s}^{\chi}\right)^{T} R\left(\Delta_{\alpha_{2} \alpha_{1}}\right)\left(\hat{L}_{s^{\prime}}^{\chi^{\prime}}\right)^{T} S\left(\Delta_{\alpha_{3} \alpha_{1}}\right)\left(\hat{L}_{s^{\prime \prime}}^{\chi^{\prime \prime}}\right)^{T}\right\}
\end{aligned}
$$

where $\Delta_{\alpha_{i} \alpha_{j}}=E_{\alpha_{i} \alpha_{j}}-\tilde{L}_{S}$, and the two functions $R(E)$ and $S(E)$ are either both symmetric or antisymmetric: $R(-E) S(-E)=R(E) S(E)$. This identity can be derived by summing over the two possibilities $\eta_{i}= \pm$ and using the representations (235), (236), (237) and (238). The functions $(R / S)\left(\Delta_{\alpha_{i} \alpha_{j}}\right)$ on the r.h.s. can be expressed in terms of the basis superoperators by using Eq. (339),

$$
\begin{aligned}
& (R / S)\left(\Delta_{\alpha_{i} \alpha_{j}}\right) \rightarrow(R / S)_{\alpha_{i} \alpha_{j}}\left(E+i \tilde{\Gamma}_{1}\right)\left(1-L^{c}\right) \\
& +(R / S)_{\alpha_{i} \alpha_{j}}\left(E-s \tilde{h}+i \tilde{\Gamma}_{2}\right) \frac{1}{2}\left(L^{c}+s L^{h}\right)
\end{aligned}
$$

where $R_{\alpha_{i} \alpha_{j}}(E)=R\left(E+\mu_{\alpha_{i}}-\mu_{\alpha_{j}}\right)$. Inserting this result in Eq. (360), we find after some straightforward algebra

$$
\begin{aligned}
\bar{G}_{12}^{(1) c} R\left(\Delta_{12}\right) \bar{G}_{\overline{2} 3}^{(1) c} S\left(\Delta_{13}\right) \bar{G}_{\overline{3} \overline{1}}^{(1) c}= & -\frac{1}{8} J_{\alpha_{1} \alpha_{2}}^{z} J_{\alpha_{2} \alpha_{3}}^{\perp} J_{\alpha_{3} \alpha_{1}}^{\perp} R_{\alpha_{1} \alpha_{2}}\left(s^{\prime} E-s \tilde{h}\right) S_{\alpha_{1} \alpha_{3}}\left(s^{\prime} E\right)\left(L^{c}+s s^{\prime} L^{h}\right) \\
& -\frac{1}{8} J_{\alpha_{1} \alpha_{2}}^{\perp} J_{\alpha_{2} \alpha_{3}}^{\perp} J_{\alpha_{3} \alpha_{1}}^{z} R_{\alpha_{1} \alpha_{2}}\left(s^{\prime} E\right) S_{\alpha_{1} \alpha_{3}}\left(s^{\prime} E-s \tilde{h}\right)\left(L^{c}+s s^{\prime} L^{h}\right) \\
& -\frac{1}{4} J_{\alpha_{1} \alpha_{2}}^{\perp} J_{\alpha_{2} \alpha_{3}}^{z} J_{\alpha_{3} \alpha_{1}}^{\perp} R_{\alpha_{1} \alpha_{2}}\left(s^{\prime} E-s \tilde{h}\right) S_{\alpha_{1} \alpha_{3}}\left(s^{\prime} E-s \tilde{h}\right)\left(L^{a}-L^{c}\right),
\end{aligned}
$$

and for $E=0$

$$
\begin{aligned}
\left.\bar{I}_{12}^{\gamma(1) c} R\left(\Delta_{12}\right) \bar{G}_{\overline{2} 3}^{(1) c} S\left(\Delta_{13}\right) \bar{G}_{\overline{3} \overline{1}}^{(1) c}\right|_{E=0}= & s \frac{1}{2} c_{\alpha_{1} \alpha_{2}}^{\gamma} J_{\alpha_{1} \alpha_{2}}^{z} J_{\alpha_{2} \alpha_{3}}^{\perp} J_{\alpha_{3} \alpha_{1}}^{\perp} R_{\alpha_{1} \alpha_{2}}(0) S_{\alpha_{1} \alpha_{3}}(s \tilde{h}) L^{1 z} \\
& +s \frac{1}{2} c_{\alpha_{1} \alpha_{2}}^{\gamma} J_{\alpha_{1} \alpha_{2}}^{\perp} J_{\alpha_{2} \alpha_{3}}^{z} J_{\alpha_{3} \alpha_{1}}^{\perp} R_{\alpha_{1} \alpha_{2}}(s \tilde{h}) S_{\alpha_{1} \alpha_{3}}(s \tilde{h}) L^{1 z}
\end{aligned}
$$

Using this result in Eq. (195), we have to distinguish many different cases in order to evaluate the logarithmic contributions according to Eq. (197). To simplify the discussion, we will assume in the following only two reservoirs with chemical potentials $\mu_{\alpha}=\alpha \frac{V}{2}$. Furthermore, we take w.l.o.g. $V, \tilde{h}>0$. As we will see in Sec. IVA, we need only the two cases $E=0$ and $E=\tilde{h}$ in Eq. (362) for the calculation of the renormalized $g$ factor and the spin relaxation and dephasing rates $\tilde{\Gamma}_{1 / 2}$ up to the first logarithmic correction. For the magnetization and the current, we consider the stationary case $E=0$.

We start with $E=0$. In this case, there are four possibilities for the parameters $|\bar{a}|$ and $|\bar{b}|$ in (197):

$$
|\bar{a}|,|\bar{b}|=\frac{V}{\Lambda_{c}}, \frac{\tilde{h}}{\Lambda_{c}}, \frac{|V \pm \tilde{h}|}{\Lambda_{c}} .
$$

According to Eq. (197), logarithmic contributions can only occur if at least one of these parameters is small compared to one. Therefore, we can distinguish three 
cases

$$
\begin{aligned}
V \ll \tilde{h} & \rightarrow \frac{V}{\Lambda_{c}} \ll 1, \\
V \sim \tilde{h} & \rightarrow \frac{|V-\tilde{h}|}{\Lambda_{c}} \ll 1, \\
V \gg \tilde{h} & \rightarrow \frac{\tilde{h}}{\Lambda_{c}} \ll 1,
\end{aligned}
$$

and all the other parameters not indicated are of order one. Discussing these three cases separately and collecting only the logarithmic terms according to Eq. (197), we find after some lengthy calculation

$$
\begin{aligned}
& \Gamma^{a(3 b)}(0)=\pi \tilde{h} \mathcal{L}_{2}(\tilde{h}) J_{\alpha}^{z}\left(J_{\alpha}^{\perp}\right)^{2} \\
& +\frac{\pi}{2}|V-\tilde{h}| \mathcal{L}_{2}(V-\tilde{h}) J_{\alpha}^{z}\left(J_{\text {nd }}^{\perp}\right)^{2} \\
& -\frac{\pi}{2}(V-\tilde{h}) \mathcal{L}_{2}(V-\tilde{h}) J_{\text {nd }}^{z} J_{\text {nd }}^{\perp} J_{\alpha}^{\perp} \\
& \Gamma^{a(3 b)}(0)+\Gamma^{c(3 b)}(0)=\frac{\pi}{2} \tilde{h} \mathcal{L}_{2}(\tilde{h}) J_{\alpha}^{z}\left(J_{\alpha}^{\perp}\right)^{2} \\
& +\frac{\pi}{4}(V-\tilde{h}+|V-\tilde{h}|) \mathcal{L}_{2}(V-\tilde{h}) J_{\text {nd }}^{z} J_{\text {nd }}^{\perp} J_{\alpha}^{\perp}, \\
& \Gamma_{\gamma}^{1 z(3 b)}(0)=-\pi V \mathcal{L}_{1}(V) J_{\text {nd }}^{\gamma z} J_{\text {nd }}^{\perp} J_{\alpha}^{\perp} \\
& -\frac{\pi}{2} \tilde{h} \mathcal{L}_{2}(\tilde{h})\left(J_{\text {nd }}^{\gamma z} J_{\text {nd }}^{\perp}+J_{\text {nd }}^{\gamma \perp} J_{\text {nd }}^{z}\right) J_{\alpha}^{\perp} \\
& +\frac{\pi}{2}|V-\tilde{h}| \mathcal{L}_{2}(V-\tilde{h}) J_{\text {nd }}^{\gamma \perp} J_{\text {nd }}^{\perp} J_{\alpha}^{z} \\
& +\frac{\pi}{4}(V-\tilde{h}) \mathcal{L}_{2}(V-\tilde{h})\left(J_{\text {nd }}^{\gamma z} J_{\text {nd }}^{\perp}-J_{\text {nd }}^{\gamma \perp} J_{\text {nd }}^{z}\right) J_{\alpha}^{\perp},
\end{aligned}
$$

with

$$
\begin{aligned}
J_{\alpha}^{z / \perp} & =J_{\alpha \alpha}^{z / \perp}, J_{\mathrm{nd}}^{z / \perp}=J_{L R}^{z / \perp}=J_{R L}^{z / \perp} \\
J_{\mathrm{nd}}^{\gamma, z / \perp} & =J_{L R}^{\gamma, z / \perp}=-J_{R L}^{\gamma, z / \perp}
\end{aligned}
$$

All other components do not contain any logarithmic contribution in third order in $J$.

Next we consider $E=\tilde{h}$. In this case, there are six possibilities for the parameters $|\bar{a}|$ and $|\bar{b}|$ in (197),

$$
|\bar{a}|,|\bar{b}|=\frac{V}{\Lambda_{c}}, \frac{\tilde{h}}{\Lambda_{c}}, \frac{|V \pm \tilde{h}|}{\Lambda_{c}}, \frac{|V \pm 2 \tilde{h}|}{\Lambda_{c}} .
$$

In addition to the three cases shown in Eq. (365), we have to consider the additional case

$$
V \sim 2 \tilde{h} \quad \rightarrow \quad \frac{|V-2 \tilde{h}|}{\Lambda_{c}} \ll 1
$$

Discussing these four cases, we find after a lengthy calculation the following logarithmic terms for the effective
Liouvillian at $E=\tilde{h}$ :

$$
\begin{aligned}
& \Gamma^{a(3 b)}(\tilde{h})=\frac{\pi}{2} \tilde{h} \mathcal{L}_{2}(\tilde{h}) J_{\alpha}^{z}\left(J_{\alpha}^{\perp}\right)^{2} \\
&+\frac{\pi}{2} V \mathcal{L}_{2}(V)\left(J_{\alpha}^{z} J_{\text {nd }}^{\perp}+J_{\text {nd }}^{z} J_{\alpha}^{\perp}\right) J_{\text {nd }}^{\perp} \\
&+\frac{\pi}{4}|V-2 \tilde{h}| \mathcal{L}_{2}(V-2 \tilde{h}) J_{\alpha}^{z}\left(J_{\text {nd }}^{\perp}\right)^{2} \\
&-\frac{\pi}{4}(V-2 \tilde{h}) \mathcal{L}_{2}(V-2 \tilde{h}) J_{\text {nd }}^{z} J_{\text {nd }}^{\perp} J_{\alpha}^{\perp}, \\
& \Gamma^{a(3 b)}(\tilde{h})+ \Gamma^{c(3 b)}(\tilde{h})= \\
&= \frac{\pi}{2} \tilde{h}\left(\frac{1}{2} \mathcal{L}_{2}(\tilde{h})+\mathcal{L}_{1}(\tilde{h})\right) J_{\alpha}^{z}\left(J_{\alpha}^{\perp}\right)^{2} \\
&-\frac{\pi}{4} \theta(\tilde{h}-V)(V-\tilde{h}) \mathcal{L}_{1}(V-\tilde{h}) J_{\alpha}^{z}\left(J_{\text {nd }}^{\perp}\right)^{2}, \\
& \operatorname{Im} h^{(3 b)}(\tilde{h})=\frac{\pi}{4} \tilde{h} \mathcal{L}_{2}(\tilde{h}) J_{\alpha}^{z}\left(J_{\alpha}^{\perp}\right)^{2} \\
& \quad-\frac{\pi}{4}(V-\tilde{h}) \mathcal{L}_{1}(V-\tilde{h}) J_{\text {nd }}^{z} J_{\text {nd }}^{\perp} J_{\alpha}^{\perp} \\
&-\frac{\pi}{4} \theta(V-\tilde{h})(V-\tilde{h}) \mathcal{L}_{1}(V-\tilde{h}) J_{\alpha}^{z}\left(J_{\text {nd }}^{\perp}\right)^{2} .
\end{aligned}
$$

\section{RESULTS}

In this section, we summarize the zero temperature results for the anisotropic nonequilibrium Kondo model in a finite magnetic field, introduced at the beginning of Sec. III A. We will calculate quantities characterizing the exponential time decay of the magnetization, i.e., the spin relaxation and dephasing rates $\tilde{\Gamma}_{1 / 2}$ together with the renormalized magnetic field $\tilde{h}$. Furthermore, we present results for the stationary current $\left\langle I^{\gamma}\right\rangle^{s t}$ and the stationary magnetization $M$. All quantities are calculated one order beyond leading order, i.e., up to the first term leading to logarithmic enhancements (suppressions) at resonance.

For simplicity, we evaluate the results for two reservoirs with voltages

$$
\mu_{\alpha}=\alpha \frac{V}{2} \quad, \quad \alpha \equiv \mathrm{L} / \mathrm{R} \equiv \pm .
$$

The diagonal and nondiagonal exchange couplings are denoted by

$$
J_{\alpha}^{z / \perp}=J_{\alpha \alpha}^{z / \perp} \quad, \quad J_{\text {nd }}^{z / \perp}=J_{L R}^{z / \perp}=J_{R L}^{z / \perp} .
$$

The various couplings are renormalized couplings evaluated at (note that the Laplace variable $E$ is fixed to either $E=0$ or $E=\tilde{h}$ in the following) the cutoff scale

$$
\Lambda_{c}=\max \{V, \tilde{h}\} .
$$

Unrenormalized (bare) couplings are denoted by the index "0". Furthermore, we consider w.l.o.g. the case $V, \tilde{h}>0$. Although our results of the previous section include all cases for the ratios between diagonal and nondiagonal couplings, we will treat in this section the realistic case where the exchange couplings fulfil the relation 
(203), i.e.,

$$
J_{\alpha \alpha^{\prime}}^{z / \perp}=2 \sqrt{x_{\alpha} x_{\alpha^{\prime}}} J^{z / \perp} \quad, \quad x_{L}+x_{R}=1 .
$$

In this case, the two couplings $J^{z}$ and $J^{\perp}$ can be calculated analytically from Eq. (274) with

$$
c^{2}=\left(J_{0}^{z}\right)^{2}-\left(J_{0}^{\perp}\right)^{2} \quad, \quad T_{K} \equiv \Lambda_{0} \sqrt{J_{0}^{\perp}}\left(\frac{J_{0}^{z}-c}{J_{0}^{z}+c}\right)^{\frac{1}{4 c}},
$$

according to Eqs. (270) and (316). Furthermore, we note that some of the third-order terms can be taken together because

$$
J_{\alpha}^{z}\left(J_{\mathrm{nd}}^{\perp}\right)^{2}=J_{\alpha}^{\perp} J_{\text {nd }}^{z} J_{\text {nd }}^{\perp}
$$

Finally, for the logarithmic terms we use the shorthand notation

$$
\mathcal{L}_{1}(x)=\ln \frac{\Lambda_{c}}{\sqrt{x^{2}+\tilde{\Gamma}_{1}^{2}}} \quad, \quad \mathcal{L}_{2}(x)=\ln \frac{\Lambda_{c}}{\sqrt{x^{2}+\tilde{\Gamma}_{2}^{2}}},
$$

and close to resonance, we use the broadened sign function

$$
\operatorname{sign}_{i}(x)=\frac{2}{\pi} \arctan \frac{x}{\tilde{\Gamma}_{i}}
$$

where $\tilde{\Gamma}_{1}\left(\tilde{\Gamma}_{2}\right)$ has to be chosen when the magnetic field does not (does) occur in $x$ (this rule refers to the case $E=0$, for $E=\tilde{h}$ also other cases can occur, see below). Furthermore, we use the broadening of the sign function also for expressions involving the absolute value or the theta function via

$$
\begin{aligned}
|x|_{i} & =x \operatorname{sign}_{i}(x), \\
\theta_{i}(x) & =\frac{1}{2}\left[1+\operatorname{sign}_{i}(x)\right] .
\end{aligned}
$$

\section{A. $g$ factor, spin relaxation and dephasing rates}

In this section, we determine the renormalized magnetic field $\tilde{h}$ or the renormalized $g$ factor

$$
\tilde{g}=2 \frac{d \tilde{h}}{d h_{0}}
$$

together with the renormalized spin relaxation and dephasing rates $\tilde{\Gamma}_{1 / 2}$. These quantities enter all formulas, especially they determine the resonance position $V=\tilde{h}$ and how the logarithmic divergencies are cut off by the rates. We note that previous works have only calculated $\tilde{\Gamma}_{1 / 2}$ up to $O\left(J^{2}\right)$ in bare perturbation theory, ${ }^{29}$ and the renormalized magnetic field has so far been calculated only up to $O(J)$, see, e.g., Ref. 33. In contrast, here we perform a renormalized perturbation theory for the rates and calculate in addition the logarithmic terms of $O\left(J^{3} \ln \right)$ for $\tilde{\Gamma}_{1 / 2}$ and those of $O\left(J^{2} \ln \right)$ for $\tilde{h}$ (corresponding to contributions in three-loop by using the conventional classification). Furthermore, we emphasize that we do not need to combine self-energy terms with vertex corrections as in slave-particle formalism 29 since we have directly set up a kinetic equation from the very beginning where the renormalized rates and the renormalized magnetic field can be directly read off by studying the poles of the reduced density matrix of the quantum dot in Laplace space, see the discussion at the end of Sec. IA We now calculate $\tilde{h}$ and $\tilde{\Gamma}_{1 / 2}$ perturbatively in the renormalized exchange couplings by using our results (340)-(342), (346)-(351), (366), (367), and (373)-(375) for $h(z)$ and $\Gamma^{a / c}(z)$ (note that we take the analytic continuation $E \rightarrow E+i \omega$ of these equations). Thereby, we consider all terms of $O\left(J^{2}, J^{3} \ln \right)$ for $\tilde{\Gamma}_{1 / 2}$, and all terms of $O\left(J^{0}\right), O(J), O\left(J^{2} \ln \right)$ for $\tilde{h}$. Terms of $O\left(J^{3}\right)\left[O\left(J^{2}\right)\right]$ without any logarithmic contribution are consistently neglected for $\tilde{\Gamma}_{1 / 2}(\tilde{h})$.

To get the perturbative solution, we insert first the lowest order results (340)- 342) into Eqs. (332) and (333). This gives

$$
\begin{aligned}
z_{1} & =-i \frac{1}{1-J_{\alpha}^{z}+\left(J_{\alpha}^{z}\right)_{0}} \Gamma^{a(2)}\left(z_{1}\right)-i \Gamma^{a(3)}\left(z_{1}\right) \\
z_{+} & =\frac{1+\frac{1}{2}\left(J_{\alpha}^{z}-\left(J_{\alpha}^{z}\right)_{0}\right)+2 c^{2} l_{c}}{1+J_{\alpha}^{z}-\left(J_{\alpha}^{z}\right)_{0}+2 c^{2} l_{c}} h_{0} \\
& +\sum_{k=2,3}\left(h^{(k)}\left(z_{+}\right)-i\left(\Gamma^{a(k)}+\Gamma^{c(k)}\right)\left(z_{+}\right)\right) .
\end{aligned}
$$

Using the forms (336) and (337) for $z_{1}$ and $z_{+}$, we see that $\tilde{\Gamma}_{1 / 2}$ starts at $O\left(J^{2}\right)$. Therefore, neglecting terms of $O\left(J^{4}\right)$, we can replace $z_{1} \rightarrow 0$ and $z_{+} \rightarrow \tilde{h}$ in the arguments of $\Gamma^{a / c(k)}$ and $h^{(k)}$ for $k=2,3$. Furthermore, we can neglect all contributions of $O\left(J^{3}\right)$ for the first term on the r.h.s. of Eq. (387), and all terms of $O\left(J^{2}\right)$ for the first term on the r.h.s. of Eq. (388) (since they do not contain any logarithmic contribution). Thus, we finally obtain

$$
\begin{aligned}
\tilde{\Gamma}_{1} & =\Gamma^{a(2)}(0)+\Gamma^{a(3)}(0) \\
\tilde{\Gamma}_{2} & =\sum_{k=2,3}\left\{-\operatorname{Im} h^{(k)}(\tilde{h})+\operatorname{Re}\left(\Gamma^{a(k)}+\Gamma^{c(k)}\right)(\tilde{h})\right\} \\
\tilde{h} & =h+\operatorname{Re} h^{(2)}(\tilde{h})+\operatorname{Im}\left(\Gamma^{a(2)}+\Gamma^{c(2)}\right)(\tilde{h})
\end{aligned}
$$

where

$$
h=\left(1-\frac{1}{2}\left(J_{\alpha}^{z}-\left(J_{\alpha}^{z}\right)_{0}\right)\right) h_{0}
$$

is the renormalized magnetic field up to first order in $J$.

Inserting the results (346)-(351), (366), (367), and (373) - (375) into Eqs. (389)-(391), and specializing to the case (376) of two reservoirs and the special form (379) of the exchange couplings, we obtain 


$$
\begin{aligned}
\tilde{\Gamma}_{1}= & \frac{\pi}{2} \tilde{h}\left(J_{\alpha}^{\perp}\right)^{2}+\frac{\pi}{2}\left(|V-\tilde{h}|_{2}+V+\tilde{h}\right)\left(J_{\text {nd }}^{\perp}\right)^{2} \\
& +\pi \tilde{h} \mathcal{L}_{2}(\tilde{h}) J_{\alpha}^{z}\left(J_{\alpha}^{\perp}\right)^{2}+\frac{\pi}{2}|V-\tilde{h}|_{2} \mathcal{L}_{2}(V-\tilde{h}) J_{\alpha}^{z}\left(J_{\text {nd }}^{\perp}\right)^{2}-\frac{\pi}{2}(V-\tilde{h}) \mathcal{L}_{2}(V-\tilde{h}) J_{\text {nd }}^{z} J_{\text {nd }}^{\perp} J_{\alpha}^{\perp}, \\
\tilde{\Gamma}_{2}= & \frac{\pi}{2} V\left(J_{\text {nd }}^{z}\right)^{2}+\frac{\pi}{4} \tilde{h}\left(J_{\alpha}^{\perp}\right)^{2}+\frac{\pi}{4}\left(|V-\tilde{h}|_{1}+V+\tilde{h}\right)\left(J_{\text {nd }}^{\perp}\right)^{2} \\
& +\frac{\pi}{2} \tilde{h} \mathcal{L}_{1}(\tilde{h}) J_{\alpha}^{z}\left(J_{\alpha}^{\perp}\right)^{2}+\frac{\pi}{4}|V-\tilde{h}|_{1} \mathcal{L}_{1}(V-\tilde{h}) J_{\alpha}^{z}\left(J_{\text {nd }}^{\perp}\right)^{2}+\frac{\pi}{4}(V-\tilde{h}) \mathcal{L}_{1}(V-\tilde{h}) J_{\text {nd }}^{z} J_{\text {nd }}^{\perp} J_{\alpha}^{\perp}, \\
\tilde{h}= & h-\frac{1}{2} \tilde{h} \mathcal{L}_{1}(\tilde{h})\left(J_{\alpha}^{\perp}\right)^{2}+\frac{1}{2}(V-\tilde{h}) \mathcal{L}_{1}(V-\tilde{h})\left(J_{\text {nd }}^{\perp}\right)^{2} .
\end{aligned}
$$

These formulas show precisely which rate cuts off the various logarithmic terms. Since the determination of the renormalized rates and the renormalized magnetic field involves also the value of the Liouvillian $L_{S}(E)$ at $E=\tilde{h}$, we can not just use the simple rule that $\tilde{\Gamma}_{1}\left(\tilde{\Gamma}_{2}\right)$ cuts off the logarithms where the magnetic field does not (does) occur (as it is the case for the stationary case $E=0$ ). In contrast, we find that the logarithmic terms of $\tilde{h}$ and $\tilde{\Gamma}_{2}$ are cut off by $\tilde{\Gamma}_{1}$, and those of $\tilde{\Gamma}_{1}$ by $\tilde{\Gamma}_{2}$.

$h$ is the well-known renormalized magnetic field up to first order in $J$ which determines the precise resonance positions. Note that the sign of the linear terms in $J$ in Eq. (392) is different from the one in Eq. (279), showing that it is important to consider the linear term in frequency of the renormalized Liouvillian to determine the correct renormalization of the magnetic field (similiar to field-theoretical rescaling techniques from the $Z$ factor $\left.{ }^{34}\right)$. Note that the renormalized magnetic field is linear in the renormalized coupling, but starts only in second order with respect to the bare coupling. Furthermore, we note that the determination of the renormalized magnetic field up to first order in $J$ is usually associated with a two-loop calculation, see, e.g., Ref. 33, i.e., the first diagram of Fig. 4 is called a two-loop diagram. We are not adopting this notation in the present paper because this diagram arises from closing the one-loop diagram for the renormalization of the vertex (the third diagram in Fig. (4). Therefore we call this diagram also one-loop and the second diagram of Fig. 4 a two-loop diagram (which usually would be classified as three-loop). We note that the classification in one-loop and two-loop contributions is not unambiguous, e.g., if one uses a not-normaled ordered version of the $\mathrm{RG}$ formalism, the renormalization of the magnetic field in lowest order in $J$ would arise from closing the renormalized vertex with itself, i.e., in this case one would classify it as one-loop. Therefore, we prefer in this paper a classification according to the orders in $J$ and not with respect to the loop topology of the diagrams.

Some insight into the logarithmic terms in $\tilde{\Gamma}_{1 / 2}$ and $\tilde{h}$ can be gained by differentiating these quantities with respect to the bare magnetic field $h_{0}$ [for the rates $\tilde{\Gamma}_{1 / 2}$ and the second-order terms in $\tilde{h}$, it is actually equivalent to differentiate with respect to $\tilde{h}$ because multiplying with $\frac{\partial \tilde{h}}{\partial h_{0}} \sim O(J)$ would just bring the expression one order higher in $J$, leading to terms which we have neglected anyhow]. Furthermore, we differentiate only the prefactor of the logarithmic terms and disregard the dependence of the renormalized exchange couplings on $h_{0}$ (which are small corrections since the logarithm varies only slowly). This gives

$$
\begin{aligned}
\frac{d \tilde{\Gamma}_{1}}{d h_{0}} & =\frac{\pi}{2}\left(J_{\alpha}^{\perp}\right)^{2}+\pi \theta_{2}(\tilde{h}-V)\left(J_{\text {nd }}^{\perp}\right)^{2}+\pi \mathcal{L}_{2}(\tilde{h}) J_{\alpha}^{z}\left(J_{\alpha}^{\perp}\right)^{2}+\pi \theta_{2}(\tilde{h}-V) \mathcal{L}_{2}(V-\tilde{h}) J_{\alpha}^{z}\left(J_{\text {nd }}^{\perp}\right)^{2} \\
\frac{d \tilde{\Gamma}_{2}}{d h_{0}} & =\frac{\pi}{4}\left(J_{\alpha}^{\perp}\right)^{2}+\frac{\pi}{2} \theta_{1}(\tilde{h}-V)\left(J_{\text {nd }}^{\perp}\right)^{2}+\frac{\pi}{2} \mathcal{L}_{1}(\tilde{h}) J_{\alpha}^{z}\left(J_{\alpha}^{\perp}\right)^{2}-\frac{\pi}{2} \theta_{1}(V-\tilde{h}) \mathcal{L}_{1}(V-\tilde{h}) J_{\alpha}^{z}\left(J_{\text {nd }}^{\perp}\right)^{2}, \\
\tilde{g}=2 \frac{d \tilde{h}}{d h_{0}} & \left.=2-\left(J_{\alpha}^{z}-\left(J_{\alpha}^{z}\right)_{0}\right)\right)-\mathcal{L}_{1}(\tilde{h})\left(J_{\alpha}^{\perp}\right)^{2}-\mathcal{L}_{1}(V-\tilde{h})\left(J_{\text {nd }}^{\perp}\right)^{2}
\end{aligned}
$$

where we have used Eq. (381) to take some terms together.

For both rates $\tilde{\Gamma}_{1 / 2}$, we get a jump in $\frac{\partial \tilde{\Gamma}_{1 / 2}}{\partial h_{0}}$ at $\tilde{h}=V$ in the leading order. In the next to leading order, there is a logarithmic enhancement for $\tilde{h} \rightarrow 0$ for both rates and a logarithmic enhancement for $\tilde{h}>V$ in $\frac{\partial \tilde{\Gamma}_{1}}{\partial h_{0}}$ while 


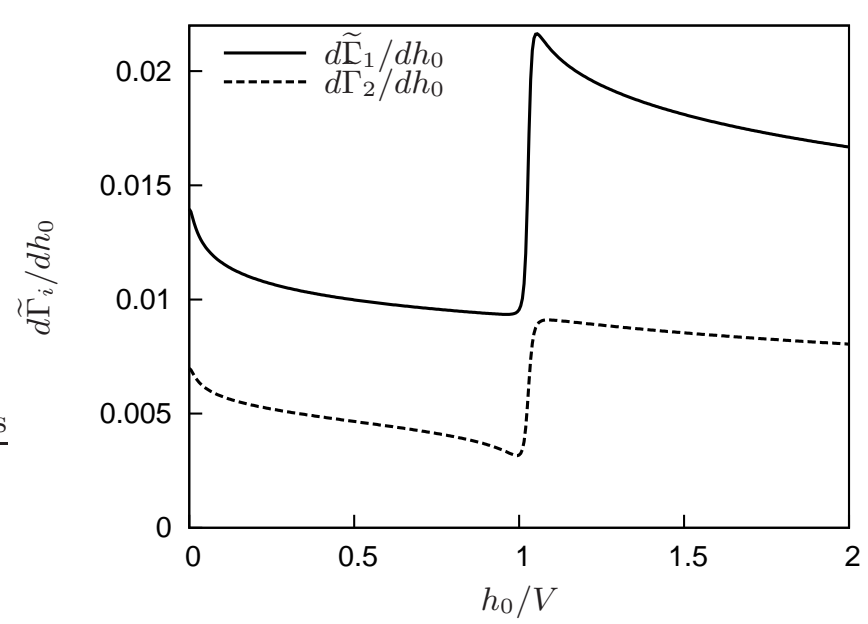

FIG. 6: The relaxation and dephasing rates $\tilde{\Gamma}_{1}$ and $\tilde{\Gamma}_{2}$, derived with respect to the magnetic field $h_{0}$, for the isotropic Kondo model with $V=10^{-4} D$ and $T_{K}=10^{-8} D$. $\frac{\partial \tilde{\Gamma}_{1}}{\partial h_{0}}$ exhibits a logarithmic enhancement for $\tilde{h}>V$ whereas $\frac{\partial \tilde{\Gamma}_{2}}{\partial h_{0}}$ is suppressed for $\tilde{h}<V$.

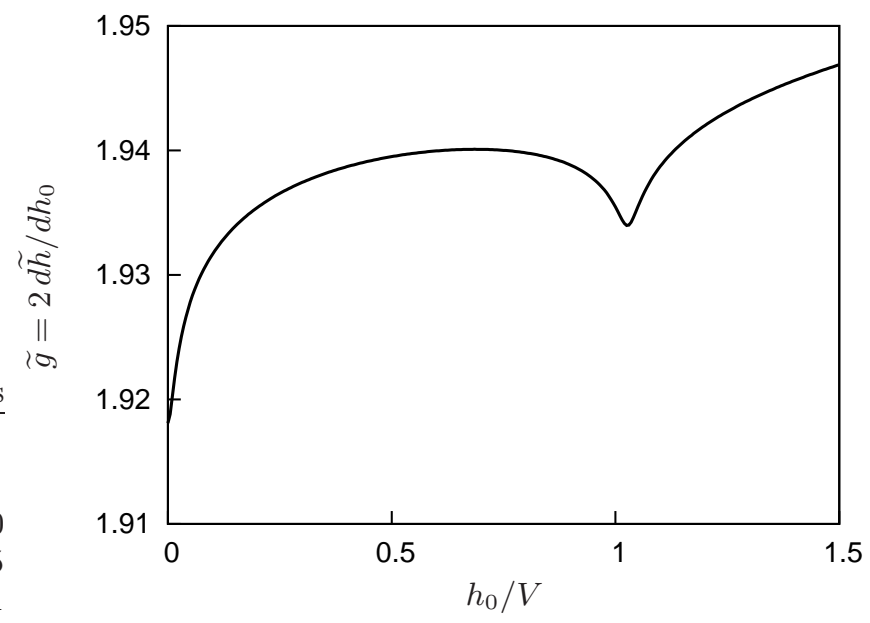

FIG. 7: $g$ factor $\tilde{g}=2 d \tilde{h} / d h_{0}$, derived with respect to the magnetic field $h_{0}$, for the isotropic Kondo model with $V=$ $10^{-4} \mathrm{D}$ and $T_{K}=10^{-8} \mathrm{D}$.

we find a logarithmic suppression in $\frac{\partial \tilde{\Gamma}_{2}}{\partial h_{0}}$ for $\tilde{h}<V$, see Fig. 6.

The renormalized $g$ factor shows a logarithmic suppression for $\tilde{h} \rightarrow 0$ and another logarithmic suppression for $\tilde{h} \approx V$ which is symmetric, in contrast to the effects we observed for the rates, see Fig. (7. As one can see, the second-order logarithmic terms lead to significant changes of the first-order shift of the $g$ factor. Especially at resonance $V=\tilde{h}$, where one usually reads off the renormalized magnetic field from the conductance (see below), the change in the $g$ factor by the logarithmic contributions is very important. However, fixing the voltage at $V=\tilde{h}$, we obtain

$$
\left.\tilde{g}\right|_{V=\tilde{h}} \approx 2-\left[J_{\alpha}^{z}-\left(J_{\alpha}^{z}\right)_{0}\right]-\left.\left(J_{\text {nd }}^{\perp}\right)^{2}\left(\ln \frac{V}{\tilde{\Gamma}_{1}}\right)\right|_{V=\tilde{h}}
$$

with

$$
\left.\left(\tilde{\Gamma}_{1}\right)\right|_{V=\tilde{h}} \approx \frac{\pi}{2} V\left[\left(J_{\alpha}^{\perp}\right)^{2}+2\left(J_{\mathrm{nd}}^{\perp}\right)^{2}\right]
$$

As a consequence, the $g$ factor at $V=\tilde{h}$ shows only a weak dependence on the bare magnetic field $h_{0}$ via the renormalization of the exchange couplings. The secondorder terms just lead to an additional overall decrease $\sim J^{2} \ln J$. To see the suppression of the $g$ factor more clearly at resonance, it is necessary to measure it directly for various values of $h_{0} / V$. In principle this can be achieved by electron spin resonance (ESR), but, similiar to previous measurements of the splitting of the Kondo resonance in the spectral density, $\stackrel{38}{,}$ we propose here a simpler setup with a weakly coupled third lead, see Fig. 8 We use the form (203) and, for simplicity, choose a symmetric coupling to the left and right leads,

$$
\begin{aligned}
& x_{L}=x_{R}=x \quad, \quad x_{P} \ll 1 \\
& J_{P, n d}^{z / \perp}=J_{P L}^{z / \perp}=J_{P R}^{z / \perp}
\end{aligned}
$$

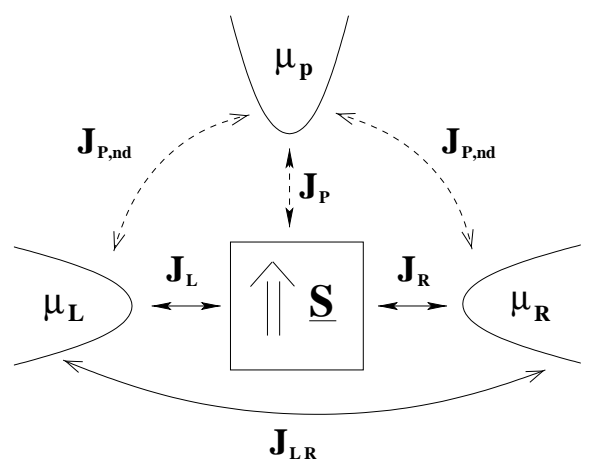

FIG. 8: Three-terminal setup including a third weakly coupled probe lead, with $\mu_{P}=e V_{P}$, in order to measure the renormalized $g$ factor as function of the applied voltage $e V=$ $\mu_{L}-\mu_{R}$.

By changing the voltage $V_{P}$ of the probe lead and measuring the differential conductance $G_{P}=\frac{d I_{P}}{d V_{P}}$, one can probe the renormalized $g$ factor as function of the voltage $V=\mu_{L}-\mu_{R}$ between the strongly coupled left and right leads. The reason is that the contribution of inelastic cotunneling to the probe current changes when the probe voltage crosses the resonance points $V_{P}=\frac{V}{2} \pm \tilde{h},-\frac{V}{2} \pm \tilde{h}$. The probe current up to second order in $J_{P, n d}$ can be calculated from Eqs. (231), (353), and (354) with the result

$$
\left\langle I^{P}\right\rangle^{s t}=\Gamma_{P}^{b(2)}(0)+2 M \Gamma_{P}^{1 z(2)}(0)
$$


where

$$
\begin{aligned}
& \Gamma_{P}^{b(2)}(0)=\frac{\pi}{4} V_{P}\left[\left(J_{P, n d}^{z}\right)^{2}+2\left(J_{P, n d}^{\perp}\right)^{2}\right], \\
& \Gamma_{P}^{1 z(2)}(0)=-\frac{\pi}{4}\left(\left|V_{P}-\frac{V}{2}-\tilde{h}\right|_{2}+\left|V_{P}+\frac{V}{2}-\tilde{h}\right|_{2}-\right. \\
& \left.-\left|V_{P}-\frac{V}{2}+\tilde{h}\right|_{2}-\left|V_{P}+\frac{V}{2}+\tilde{h}\right|_{2}\right)\left(J_{P, n d}^{\perp}\right)^{2}
\end{aligned}
$$

Here, $M$ is the magnetization, which in lowest order is independent of the probe lead and is calculated in the next section. Taking the derivative with respect to $V_{P}$, we find that $\frac{d \Gamma_{P}^{b(2)}(0)}{d V_{P}}$ is independent of $V_{P}$, whereas $\frac{d \Gamma_{P}^{1 z(2)}(0)}{d V_{P}}$ gives rise to a steplike structure shown in Figs. 9 and 10 for the two regimes $V>2 \tilde{h}$ and $V<2 \tilde{h}$, respectively. Sufficiently far away from resonance, we obtain analytically from Eq. (405) for $V>2 \tilde{h}$

$\frac{d \Gamma^{1 z(2)}}{d V_{P}}=\frac{\pi}{2}\left(J_{P, n d}^{\perp}\right)^{2} \begin{cases}1, & \text { for } \frac{V}{2}+\tilde{h}>V_{P}>\frac{V}{2}-\tilde{h} \\ & \text { or }-\frac{V}{2}+\tilde{h}>V_{P}>-\frac{V}{2}-\tilde{h} \\ 0, & \text { otherwise, }\end{cases}$

and for $V<2 \tilde{h}$

$\frac{d \Gamma^{1 z(2)}}{d V_{P}}=\frac{\pi}{2}\left(J_{P, n d}^{\perp}\right)^{2} \begin{cases}2, & \text { for }-\frac{V}{2}+\tilde{h}>V_{P}>\frac{V}{2}-\tilde{h} \\ 1, & \text { for } \frac{V}{2}+\tilde{h}>V_{P}>-\frac{V}{2}+\tilde{h} \\ & \text { or } \frac{V}{2}-\tilde{h}>V_{P}>-\frac{V}{2}-\tilde{h} \\ 0, & \text { otherwise. }\end{cases}$

From the width of the steplike features one can read off the renormalized magnetic field $\tilde{h}$ at given voltage $V$ and magnetic field $h_{0}$. Thus, by varying $V$ or $h_{0}$, one can measure the renormalized $g$ factor as function of $h_{0} / V$, thereby revealing the suppression at resonance $V=\tilde{h}$ as shown in Fig. 7. We note that the experimental situation is usually in the strong coupling regime $J \sim O(1)$, where the qualitative features are expected to be more pronounced.

\section{B. Magnetization and susceptibility}

The stationary magnetization (which is nonzero only if a magnetic field $h_{0} \neq 0$ is applied) can be calculated from the stationary density matrix (230). This density matrix is the solution of the kinetic equation and is a diagonal matrix. The occupation probabilities for the states $|\uparrow\rangle$ and $\downarrow\rangle$ are

$$
p_{\uparrow}=\frac{\Gamma^{a}(0)-\Gamma^{3 z}(0)}{2 \Gamma^{a}(0)} \quad, \quad p_{\downarrow}=\frac{\Gamma^{a}(0)+\Gamma^{3 z}(0)}{2 \Gamma^{a}(0)},
$$

and the magnetization is given by

$$
M=\frac{1}{2}\left(p_{\uparrow}-p_{\downarrow}\right)=-\frac{\Gamma^{3 z}(0)}{2 \Gamma^{a}(0)} .
$$

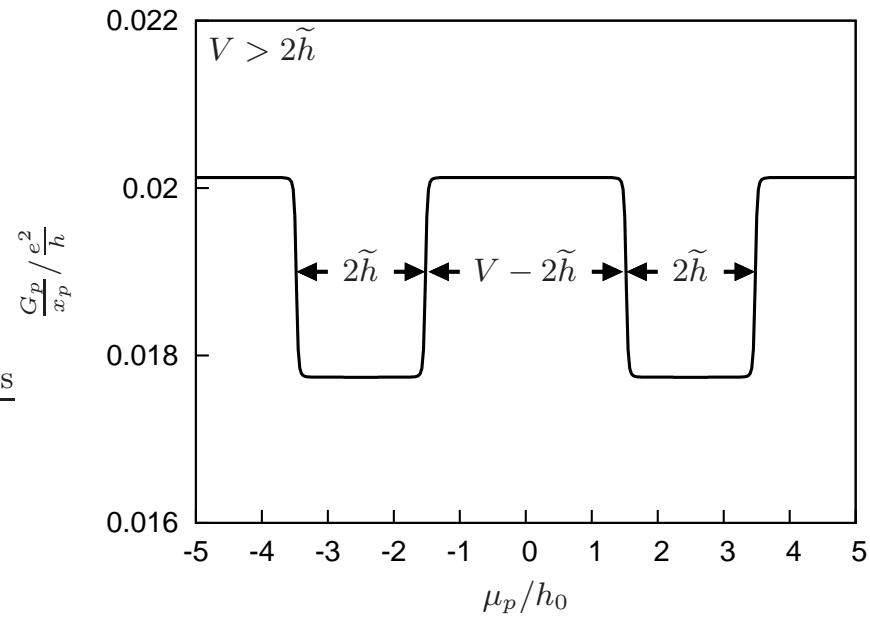

FIG. 9: Probe-lead conductance in a three-terminal setup for $h_{0}=10^{-4} D, V=5 \times 10^{-4} D$, and $T_{K}=10^{-8} D$.

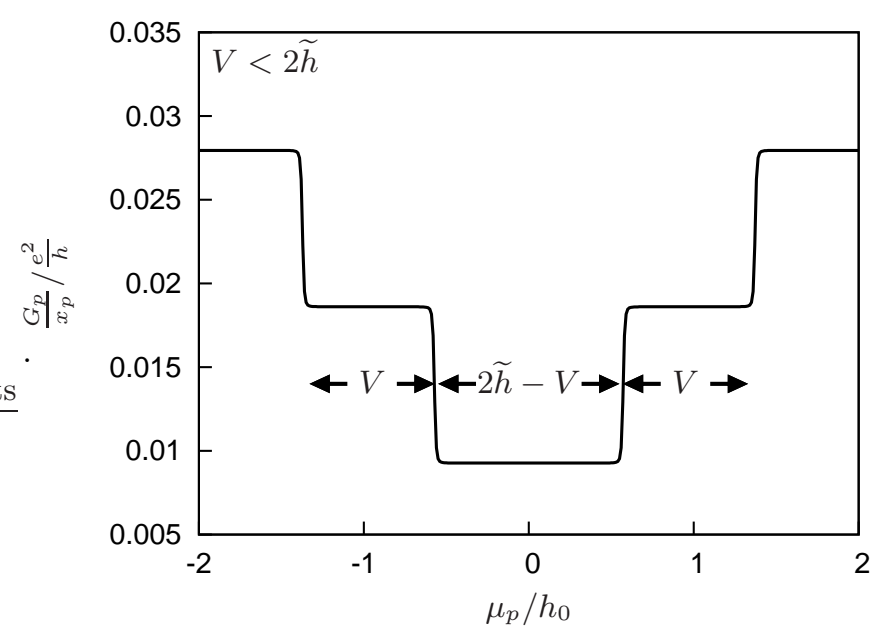

FIG. 10: Probe-lead conductance in a three-terminal setup for $h_{0}=10^{-4} D, V=8 \times 10^{-5} D$, and $T_{K}=10^{-8} D$.

$\Gamma^{a}(0)=\tilde{\Gamma}_{1}$ is given by Eq. (393)..$\Gamma^{3 z}(0)$ can be calculated from Eqs. (352) and (358):

$$
\begin{aligned}
& \Gamma^{3 z}(0)=\frac{\pi}{2} \tilde{h}\left\{\left(J_{\alpha}^{\perp}\right)^{2}+2\left(J_{\text {nd }}^{\perp}\right)^{2}\right\} \\
& +\pi \tilde{h} \mathcal{L}_{2}(\tilde{h})\left\{J_{\alpha}^{z}\left(J_{\alpha}^{\perp}\right)^{2}+J_{\alpha}^{\perp} J_{\text {nd }}^{z} J_{\text {nd }}^{\perp}\right\} \\
& \quad-\frac{\pi}{2}(V-\tilde{h}) \mathcal{L}_{2}(V-\tilde{h})\left\{J_{\alpha}^{z}\left(J_{\text {nd }}^{\perp}\right)^{2}+J_{\alpha}^{\perp} J_{\text {nd }}^{z} J_{\text {nd }}^{\perp}\right\},
\end{aligned}
$$

where we have replaced $h_{0} \rightarrow \tilde{h}$ in the first term, which gives only rise to negligible terms of $O\left(J^{3}, J^{4} \ln \right)$. Because we have considered all terms of $O\left(J^{2}, J^{3} \ln \right)$ for the rates, we can calculate the leading order $O\left(J^{0}\right)$ (which is independent of the couplings) and logarithmically enhanced terms in $O(J \ln )$ of the magnetization.

Using Eq. (381) and the abbreviation

$$
\begin{aligned}
X= & \tilde{h}\left(J_{\alpha}^{\perp}\right)^{2}+2 \tilde{h}\left(J_{\mathrm{nd}}^{\perp}\right)^{2}+2 \tilde{h} \mathcal{L}_{2}(\tilde{h}) J_{\alpha}^{z}\left(J_{\alpha}^{\perp}\right)^{2} \\
& -2(V-\tilde{h}) \mathcal{L}_{2}(V-\tilde{h}) J_{\alpha}^{z}\left(J_{\mathrm{nd}}^{\perp}\right)^{2},
\end{aligned}
$$


we can write the magnetization in the compact form

$$
\begin{aligned}
& M=-\frac{1}{2} \cdot \\
& \cdot \frac{X+2 \tilde{h} \mathcal{L}_{2}(\tilde{h}) J_{\alpha}^{z}\left(J_{\text {nd }}^{\perp}\right)^{2}}{X+\left(|V-\tilde{h}|_{2}+V-\tilde{h}\right)\left(1+\mathcal{L}_{2}(V-\tilde{h}) J_{\alpha}^{z}\right)\left(J_{\text {nd }}^{\perp}\right)^{2}} .
\end{aligned}
$$

We note that, in the isotropic case, and when expanded systematically in $J$, the terms of $O\left(J^{0}, J \ln \right)$ are in agreement with the perturbative results obtained in Ref. 32 and the poor man scaling results of Ref. 20 if the different definition of the magnetization is taken into account (the magnetization in those references is $M=1$ in the ground state, whereas it is $M=-1 / 2$ here). However, we have shown here how the spin dephasing rate cuts off all the logarithmic divergencies (the treatment in Ref. 29 was only perturbative in the bare coupling and did not treat the case of finite magnetic field).

If the voltage is smaller than the renormalized magnetic field $\tilde{h}$, the logarithmic term $\sim \mathcal{L}_{2}(\tilde{h})$ in the numerator of Eq. (412) must be consistently neglected and the magnetization is equal to $-1 / 2$. This is because the energy difference provided by the voltage is insufficient to flip the spin $1 / 2$ in the dot out of its ground state $|\downarrow\rangle$.

In the limit $V \gg \tilde{h}$, the logarithmic term $\sim \mathcal{L}_{2}(V-\tilde{h})$ in the denominator of Eq. (412) must be neglected and the magnetization is given by

$$
M=-\frac{1}{2} \frac{X+2 \tilde{h} \mathcal{L}_{2}(\tilde{h}) J_{\alpha}^{z}\left(J_{\text {nd }}^{\perp}\right)^{2}}{X+2 V\left(J_{\text {nd }}^{\perp}\right)^{2}},
$$

which, for $J_{\text {nd }}^{z / \perp} \sim J_{\alpha}^{z / \perp}$, can be expanded as

$$
\begin{aligned}
M= & -\frac{\tilde{h}}{2 V}\left\{1+\frac{1}{2}\left(\frac{J_{\alpha}^{\perp}}{J_{\text {nd }}^{\perp}}\right)^{2}+\right. \\
& \left.+\mathcal{L}_{2}(\tilde{h}) J_{\alpha}^{z}\left(1+\left(\frac{J_{\alpha}^{\perp}}{J_{\text {nd }}^{\perp}}\right)^{2}\right)\right\} .
\end{aligned}
$$

As we see, there is an interesting logarithmic enhancement at $\tilde{h}=0$, which is a pure nonequilibrium effect [if we set $J_{\text {nd }}^{\perp}=0$ in Eq. (413), we get $\left.M=-\frac{1}{2}\right]$.

In the limit $V>\tilde{h}, V-\tilde{h} \ll \tilde{h}$, (i.e., for voltages which are slightly larger than the renormalized magnetic field), we can neglect all logarithmic terms $\sim \mathcal{L}_{2}(\tilde{h})$ and find after expanding in $J$

$$
\begin{aligned}
& M \approx-\frac{1}{2}+\frac{V-\tilde{h}}{\tilde{h}} \frac{\left(J_{\mathrm{nd}}^{\perp}\right)^{2}}{\left(J_{\mathrm{L}}^{\perp}+J_{\mathrm{R}}^{\perp}\right)^{2}} . \\
& \cdot\left[1+\left(J_{\mathrm{L}}^{z}+J_{\mathrm{R}}^{z}\right) \mathcal{L}_{2}(V-\tilde{h})\right] .
\end{aligned}
$$

This result shows the logarithmic enhancement of the magnetization slightly above the resonance.

From the magnetization, we can also calculate the susceptibility

$$
\chi=\frac{\partial M}{\partial h_{0}} \approx \frac{\partial M}{\partial \tilde{h}}
$$

which we calculate by differentiating the magnetization (409) with respect to the bare magnetic field $h_{0}$ [or the renormalized field $\tilde{h}$ by multiplying with $\frac{d \tilde{h}}{d h_{0}}=$ $\left.1+O\left(J, J^{2} \ln \right)\right]$. In the special case of symmetric couplings $\left(J_{\mathrm{L}}^{\chi}=J_{\mathrm{R}}^{\chi}=J_{\text {nd }}^{\chi}\right.$ for $\left.\chi=z, \perp\right)$, the magnetization for $V>\tilde{h}$ can be expanded as

$$
\begin{aligned}
M= & -\frac{\tilde{h}}{V+\tilde{h}}+\frac{V-\tilde{h}}{V+\tilde{h}} \mathcal{L}_{2}(V-\tilde{h}) J^{z} \\
& -\frac{2 V \tilde{h}}{(V+\tilde{h})^{2}} \mathcal{L}_{2}(\tilde{h}) J^{z},
\end{aligned}
$$

and the susceptibility becomes

$$
\begin{aligned}
\chi= & -\frac{V}{(V+\tilde{h})^{2}}-\frac{2 V}{(V+\tilde{h})^{2}} \mathcal{L}_{2}(V-\tilde{h}) J^{z} \\
& -\frac{2 V(V-\tilde{h})}{(V+\tilde{h})^{3}} \mathcal{L}_{2}(\tilde{h}) J^{z} .
\end{aligned}
$$

To visualize the logarithmic effects better, we multiply $\chi$ with $-(V+\tilde{h})^{2} / V$ and get

$$
-\frac{(V+\tilde{h})^{2}}{V} \chi=1+2 J^{z} \mathcal{L}_{2}(V-\tilde{h})+2 \frac{V-\tilde{h}}{V+\tilde{h}} J^{z} \mathcal{L}_{2}(h) .
$$

Plotting Eq. (419) as function of the magnetic field $h_{0}$ emphasizes the logarithmic enhancements at $\tilde{h} \ll V$ and $\tilde{h} \approx V$, see Fig. 11] In this plot, we multiply the suscep-

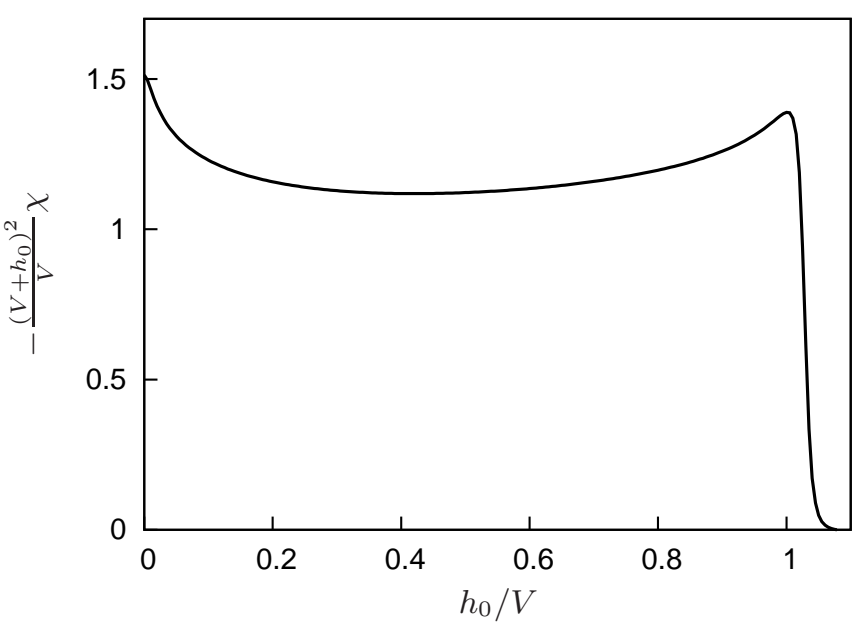

FIG. 11: The susceptibility $\chi$, multiplied with $-\frac{\left(V+h_{0}\right)^{2}}{V}$, as function of the magnetic field $h_{0}$ for the isotropic Kondo model with $V=10^{-4} D$ and $T_{K}=10^{-8} D$.

tibility $\chi$ not with $-\frac{(V+\tilde{h})^{2}}{V}$, but with $-\frac{\left(V+h_{0}\right)^{2}}{V}$ because this change causes only subleading corrections and because the unrenormalized magnetic field $h_{0}$ is easier to access experimentally.

In summary, from the various expansions Eqs. (414), (415), and (417), we find that, for $V>\tilde{h}$, the logarithmic terms at $\tilde{h}=0$ and $\tilde{h}=V$ are essentially proportional to 
the longitudinal exchange coupling $J^{z}$. This means that even in the limit $J^{\perp} \rightarrow 0$, these logarithmic contributions survive, which is an interesting nonequilibrium effect.

\section{Current}

The stationary current can be calculated from Eq. (231),

$$
\left\langle I^{\gamma}\right\rangle^{s t}=\Gamma_{\gamma}^{b}(0)+2 M \Gamma_{\gamma}^{1 z}(0),
$$

where the stationary magnetization $M$ follows from Eq. (409) and the components $\Gamma_{\gamma}^{b}(0)$ and $\Gamma_{\gamma}^{1 z}(0)$ of the current kernel are listed in Eqs. (353), (354), (359), and (368). For the special case (376) of two reservoirs and the relation (379) between the exchange couplings, we obtain

$$
\begin{aligned}
& \Gamma_{\gamma}^{b}(0)= \frac{\pi}{4} \gamma V\left\{\left(J_{\text {nd }}^{z}\right)^{2}+2\left(J_{\text {nd }}^{\perp}\right)^{2}\right\} \\
&+\frac{\pi}{2} \gamma V \mathcal{L}_{1}(V) J_{\text {nd }}^{z} J_{\alpha}^{\perp} J_{\text {nd }}^{\perp} \\
&+\frac{\pi}{4} \gamma(V-\tilde{h}) \mathcal{L}_{2}(V-\tilde{h}) J_{\text {nd }}^{\perp}\left(J_{\alpha}^{z} J_{\text {nd }}^{\perp}+J_{\alpha}^{\perp} J_{\text {nd }}^{z}\right), \\
& \Gamma_{\gamma}^{1 z}(0)=-\frac{\pi}{4} \gamma\left(|V-\tilde{h}|_{2}-V-\tilde{h}\right)\left(J_{\text {nd }}^{\perp}\right)^{2} \\
& \quad+\frac{\pi}{2} \gamma\left(V \mathcal{L}_{1}(V)+\tilde{h} \mathcal{L}_{2}(\tilde{h})\right) J_{\text {nd }}^{z} J_{\alpha}^{\perp} J_{\text {nd }}^{\perp} \\
& \quad-\frac{\pi}{4} \gamma|V-\tilde{h}|_{2} \mathcal{L}_{2}(V-\tilde{h}) J_{\alpha}^{z}\left(J_{\text {nd }}^{\perp}\right)^{2},
\end{aligned}
$$

where we have used $c_{\alpha \alpha^{\prime}}^{\gamma}=-\frac{1}{2} \alpha \gamma \delta_{\alpha,-\alpha^{\prime}}$.

For $V \ll \tilde{h}$, the logarithmic terms $\sim \mathcal{L}_{2}(\tilde{h}), \mathcal{L}_{2}(V-\tilde{h})$ can be neglected and the magnetization is given by $M=-\frac{1}{2}$. According to Eq. (420), this leads to $\left\langle I^{\gamma}\right\rangle^{s t}=$ $\Gamma_{\gamma}^{b}(0)-\Gamma_{\gamma}^{1 z}(0)$, and we see that the logarithmic terms $\sim \mathcal{L}_{1}(V)$ cancel. This gives the result of elastic cotunneling

$$
\left\langle I^{\gamma}\right\rangle^{s t}=\frac{\pi}{4} \gamma V\left(J_{\text {nd }}^{z}\right)^{2},
$$

but with renormalized exchange couplings.

At $V \approx \tilde{h}$, the differential conductance $G_{\gamma}=\frac{d I_{\gamma}}{d V}$ has an interesting feature, see Fig. 12, For voltages slightly below the renormalized magnetic field $(V<\tilde{h}, \tilde{h}-V \ll$ $\tilde{h}$ ), we get in units of $G_{0}=e^{2} / h$ (as before, we list the leading order and logarithmic terms in next-to-leading order)

$$
G_{\gamma} / G_{0}=\gamma \frac{\pi^{2}}{2}\left[\left(J_{\text {nd }}^{z}\right)^{2}+\left(J_{\mathrm{L}}^{z}+J_{\mathrm{R}}^{z}\right)\left(J_{\text {nd }}^{\perp}\right)^{2} \mathcal{L}_{2}(V-\tilde{h})\right] .
$$

For voltages slightly above the renormalized magnetic field $(V>\tilde{h}, V-\tilde{h} \ll \tilde{h})$, we get

$$
\begin{aligned}
& G_{\gamma} / G_{0}=\gamma \frac{\pi^{2}}{2}\left\{\left(J_{\text {nd }}^{z}\right)^{2}+\left(J_{\text {nd }}^{\perp}\right)^{2}\left[2+\left(\frac{2 J_{\text {nd }}^{\perp}}{J_{\mathrm{L}}^{\perp}+J_{\mathrm{R}}^{\perp}}\right)^{2}\right]\right. \\
& \left.+\left(J_{\mathrm{L}}^{z}+J_{\mathrm{R}}^{z}\right)\left(J_{\mathrm{nd}}^{\perp}\right)^{2}\left[3+\left(\frac{2 J_{\mathrm{nd}}^{\perp}}{J_{\mathrm{L}}^{\perp}+J_{\mathrm{R}}^{\perp}}\right)^{2}\right] \mathcal{L}_{2}(V-\tilde{h})\right\} .
\end{aligned}
$$

Two interesting features happen at $V \approx \tilde{h}$ : There is a jump in the leading order term in the conductance (if $J_{\text {nd }}^{\perp} \neq 0$ ) that is due to inelastic cotunneling which sets in at this voltage. The jump is superposed by a logarithmic term which becomes largest for $V=\tilde{h}$. The experimentally accessible parameters characterizing the line shape are given by the position, broadening and height of the resonance. The position is approximately at $V=\tilde{h}$ [up to unimportant terms of the $\left.O\left(\tilde{\Gamma}_{2}\right)\right]$, and the broadening of the left side of the resonance is given by $\tilde{\Gamma}_{2}$. Using Eqs. (395) and (394), we obtain at resonance

$$
\begin{aligned}
&\left.\tilde{h}\right|_{V=\tilde{h}} \approx h \\
&\left.\tilde{\Gamma}_{2}\right|_{V=\tilde{h}} \approx \frac{\pi}{2} V\left\{\left(J_{\text {nd }}^{z}\right)^{2}+\left(J_{\text {nd }}^{\perp}\right)^{2}+\frac{1}{2}\left(J_{\alpha}^{\perp}\right)^{2}\right\} .
\end{aligned}
$$

The right side of the resonance has no characteristic broadening: asymptotically it reveals the logarithmic voltage dependence of the exchange couplings. The value of the conductance at resonance can be calculated in good approximation by taking the extrapolation of Eq. (425) at $V=\tilde{h}$, i.e., by replacing $\mathcal{L}_{2}(V-\tilde{h}) \rightarrow \ln \frac{V}{\left.\Gamma_{2}\right|_{V=\tilde{h}}}$ in Eq. (425). Together with Eq. (427), this reveals the Kondo-induced logarithmic enhancement $\sim J^{3} \ln J$ of the conductance at resonance superposed on the enhancement from inelastic cotunneling. The result for the maximal conductance can be simplified by using the relation (379), which gives

$$
\begin{aligned}
&\left(G_{\gamma} / G_{0}\right)_{\max } \approx \gamma 2 \pi^{2} x_{L} x_{R} \\
& \cdot\left\{\left(J^{z}\right)^{2}+2\left(1+2 x_{L} x_{R}\right)\left(J^{\perp}\right)^{2}+\right. \\
&\left.\quad+2\left(3+4 x_{L} x_{R}\right) J^{z}\left(J^{\perp}\right)^{2} \ln \frac{V}{\left.\tilde{\Gamma}_{2}\right|_{V=\tilde{h}}}\right\}
\end{aligned}
$$

with

$$
\left.\tilde{\Gamma}_{2}\right|_{V=\tilde{h}}=\pi V\left\{2 x_{L} x_{R}\left(J^{z}\right)^{2}+\left(J^{\perp}\right)^{2}\right\} .
$$

This result for the value of the maximum conductance at resonance has first been noted in Ref. 19 for the case of the isotropic Kondo model and $x_{L}=x_{R}=\frac{1}{2}$.

Our results for the differential conductance agree with those of Refs. 20 and 19. However, note that the precise line shape at resonance can only be obtained if one includes the cutoff scales $\tilde{\Gamma}_{1 / 2}$ into the logarithms and uses the correct smearing (383) of the sign function. In the numerical plots, we have always used these smeared forms and we calculated $\tilde{h}$ and $\tilde{\Gamma}_{1 / 2}$ self-consistenly from Eqs. (393)-(395) (the same procedure has been used for the susceptibility).

\section{Results for the anisotropic Kondo model}

In this section, we discuss how the results for the differential conductance, the susceptibility, the relaxation 


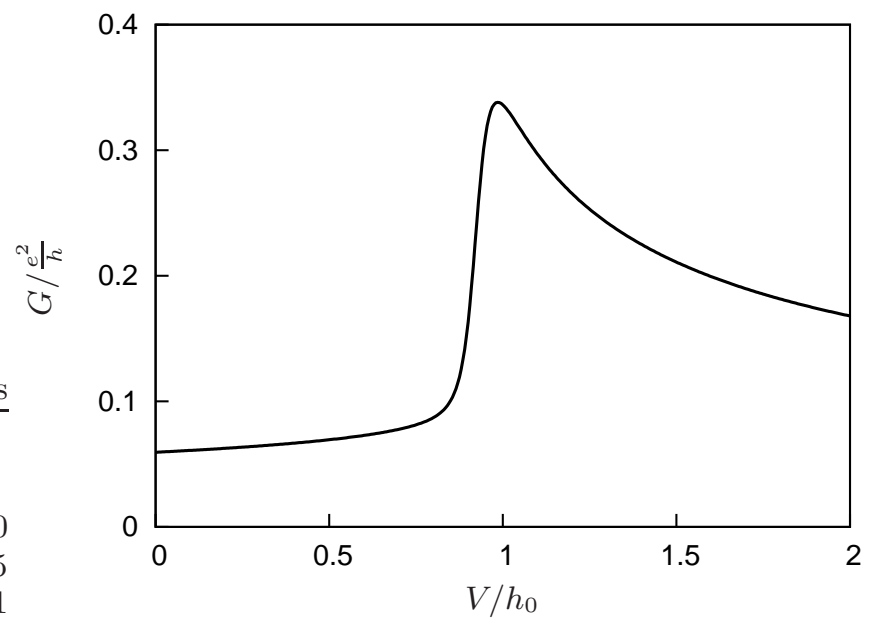

FIG. 12: The differential conductance $G=\frac{d I}{d V}$ as function of bias voltage at $h_{0}=10^{-4} D$ for the isotropic Kondo model with $T_{K}=10^{-6} \mathrm{D}$.

and dephasing rates, and the renormalized $g$ factor are affected if we consider the symmetric, but anisotropic Kondo model with $J_{\alpha \alpha^{\prime}}^{z}=J^{z} \neq J^{\perp}=J_{\alpha \alpha^{\prime}}^{\perp}$. As recently proposed,, 21 such models can, e.g., be experimentally realized by studying quantum transport through single molecular magnets, where the transverse exchange coupling is induced by magnetic quantum tunneling terms due to transverse anisotropies. To investigate this, we vary $J^{z}$ and $J^{\perp}$ such that the Kondo temperature $T_{K}$ remains constant and only the other invariant of the RG equations, i.e., $c^{2}=\left(J^{z}\right)^{2}-\left(J^{\perp}\right)^{2}$ is changed, see (270). If $c^{2}$ is positive, $J^{z}$ is larger and $J^{\perp}$ is smaller than the isotropic coupling in the case $c^{2}=0$.

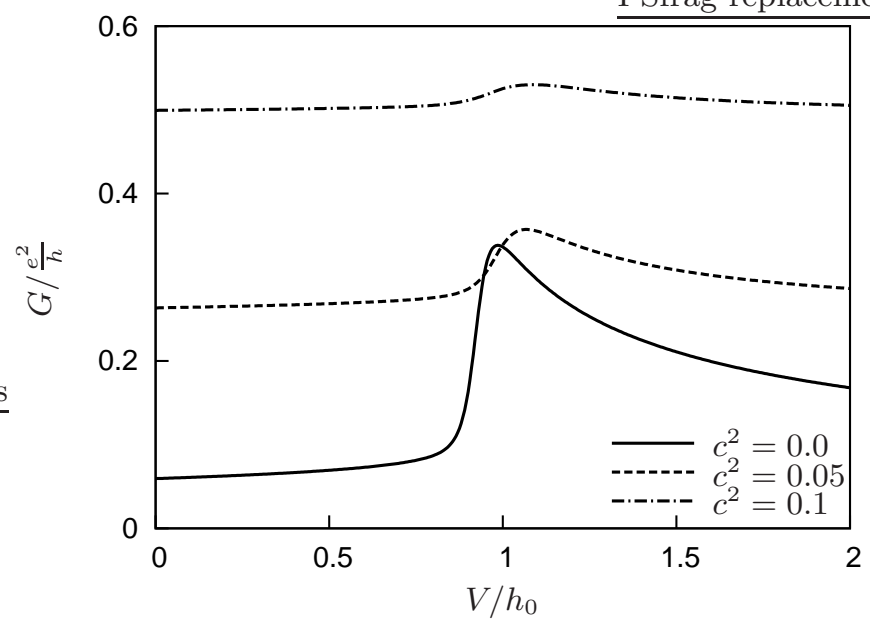

FIG. 13: The differential conductance $G=\frac{d I}{d V}$ as function of bias voltage at $h_{0}=10^{-4} D$ for the isotropic Kondo model (solid line) and the anisotropic Kondo model with two different values of $c^{2}=\left(J^{z}\right)^{2}-\left(J^{\perp}\right)^{2}$ (dashed and dash-dotted lines). The Kondo temperature $T_{K}=10^{-6} D$ is the same in all cases.
The differential conductance is shown for three different values of $c^{2}$ in Fig. 13], For growing $c^{2}$, i.e., increasing $J^{z}$ and decreasing $J^{\perp}$, we can observe a number of different effects compared to the isotropic case: First, the conductance for $V \ll \tilde{h}$ which is proportional to $\left(J_{\text {nd }}^{z}\right)^{2}$ according to Eq. (423) is increased. Second, the step at $V=\tilde{h}$ that is due to inelastic cotunneling decreases its height which is proportional to $\left(J_{\text {nd }}^{\perp}\right)^{2}$ in leading order according to Eqs. (424) and (425). Third, also the logarithmic enhancement at $V=\tilde{h}$ is reduced for increasing $c^{2}$. The prefactors of the logarithmic terms in Eqs. (424) and (425) are proportional to $J^{z}\left(J^{\perp}\right)^{2}$, a quantity which decreases for increasing $c^{2}$. Finally, the position of the step and the logarithmic enhancement shifts to the right, i.e., $\tilde{h}$ is increased. The reason is that the leading contribution to $\tilde{h}-h_{0}$ is negative and proportional to $J_{\alpha}^{z}-\left(J_{\alpha}^{z}\right)_{0}$, i.e., the renormalization of the coupling $J^{z}$, according to Eq. (392). This renormalization is in leading order determined by $\left(J_{\text {nd }}^{\perp}\right)^{2}$, see Eq. (266). An increase in $c^{2}$ which is connected to a decrease in $J^{\perp}$ therefore leads to a weaker renormalization of $J^{z}$ and hence to a larger value of $\tilde{h}$.

Figure 14 shows the magnetic susceptibility $\chi=\frac{d M}{d h_{0}}$, multiplied with $-\frac{\left(V+h_{0}\right)^{2}}{V}$, for different values of the anisotropy. For larger $c^{2}$, i.e., increased $J^{z}$, the logarith-

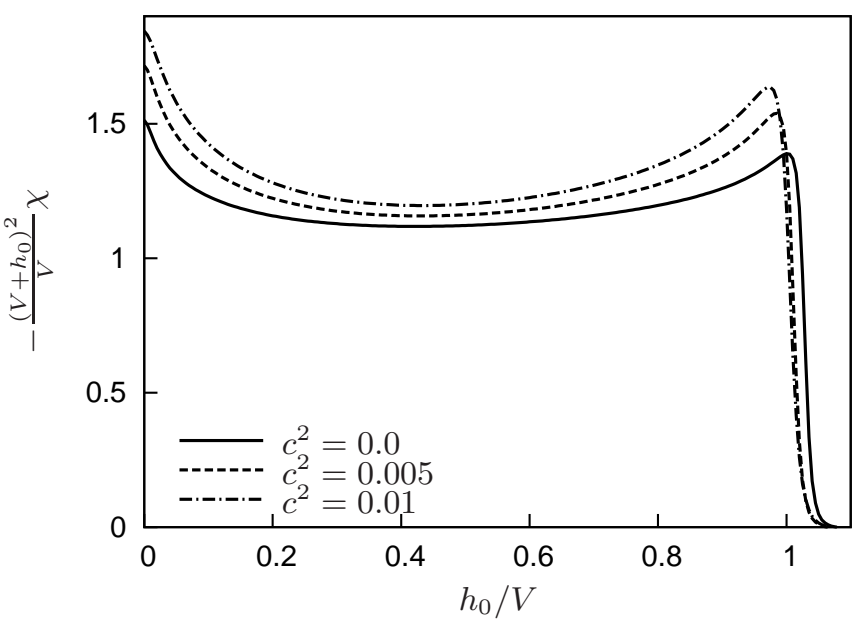

FIG. 14: The susceptibility $\chi$, multiplied with $-\frac{\left(V+h_{0}\right)^{2}}{V}$, as function of the magnetic field at $V=10^{-4} D$ for the isotropic Kondo model (solid line) and the anisotropic Kondo model with two different values of $c^{2}=\left(J^{z}\right)^{2}-\left(J^{\perp}\right)^{2}$ (dashed and dash-dotted lines). The Kondo temperature $T_{K}=10^{-8} D$ is the same in all cases.

mic enhancements become larger, see Eq. (419). This is an effect already mentioned at the end of Sec. IVB. The susceptibility depends only on the longitudinal coupling $J^{z}$ and the logarithmic contributions survive even in the limit $J^{\perp} \rightarrow 0$. Especially for molecular magnets with a very small quantum tunneling term, this might be an interesting possibility to observe logarithmic contributions in the susceptibility although the Kondo temperature might be quite small. Furthermore, the resonance 
at the point where $\tilde{h}=V$ shifts slightly, this is due to the dependence of $\tilde{h}$ on the anisotropy which was discussed above.

The derivatives of the rates $\tilde{\Gamma}_{1}$ and $\tilde{\Gamma}_{2}$ with respect to the magnetic field $h_{0}$ are shown in Figs. 15] and 16, respectively. Increasing $c^{2}$, i.e., decreasing $J^{\perp}$, leads to a

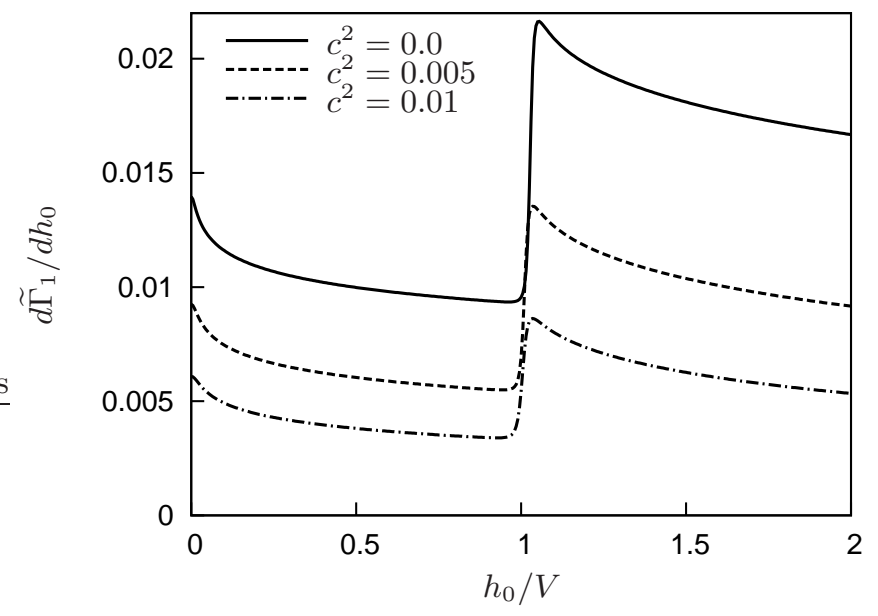

FIG. 15: The rate $\tilde{\Gamma}_{1}$, derived with respect to the magnetic field, as function of the magnetic field at $V=10^{-4} \mathrm{D}$ for the isotropic Kondo model (solid line) and the anisotropic Kondo model with two different values of $c^{2}=\left(J^{z}\right)^{2}-\left(J^{\perp}\right)^{2}$ (dashed and dash-dotted lines). The Kondo temperature $T_{K}=10^{-8} \mathrm{D}$ is the same in all cases.

decrease in the leading-order contributions to the derivatives of the rates which are proportional to $\left(J_{\mathrm{nd}}^{\perp}\right)^{2}$, see Eqs. (396) and (397). The dependence of the logarithmic

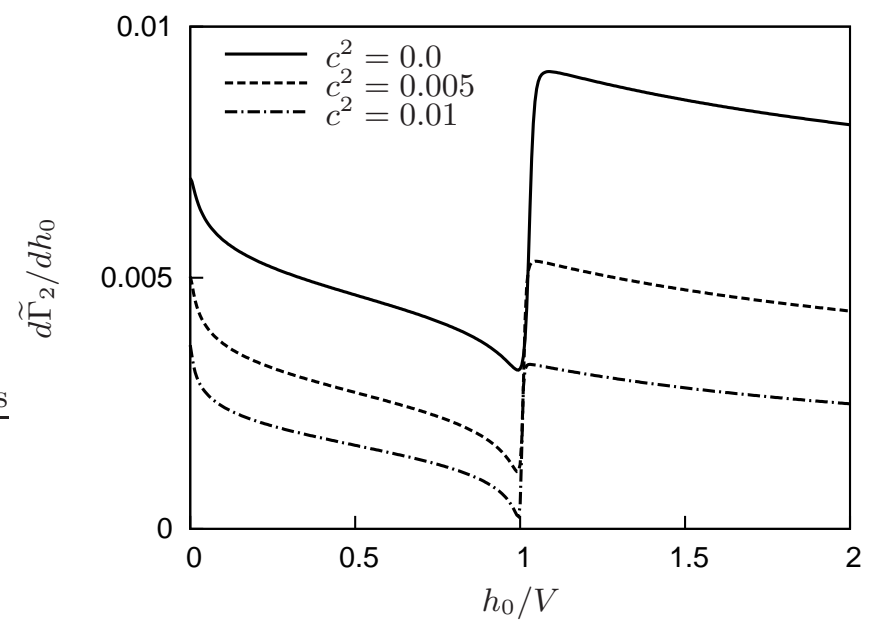

FIG. 16: The rate $\tilde{\Gamma}_{2}$, derived with respect to the magnetic field, as function of the magnetic field at $V=10^{-4} \mathrm{D}$ for the isotropic Kondo model (solid line) and the anisotropic Kondo model with two different values of $c^{2}=\left(J^{z}\right)^{2}-\left(J^{\perp}\right)^{2}$ (dashed and dash-dotted lines). The Kondo temperature $T_{K}=10^{-8} \mathrm{D}$ is the same in all cases.

effects at $\tilde{h}=0$ and $\tilde{h}=V$ on the anisotropy is different for the derivatives of the two different rates: The features in $d \tilde{\Gamma}_{1} / d h_{0}$ become less pronounced whereas the resonances in $d \tilde{\Gamma}_{2} / d h_{0}$ become sharper. The reason is as follows: all logarithmic terms in next to leading order in the rates are proportional to $J^{z}\left(J_{\text {nd }}^{\perp}\right)^{2}$ which decreases for larger anisotropy, but the logarithms are broadened by $\tilde{\Gamma}_{2}$ in the case of $d \tilde{\Gamma}_{1} / d h_{0}$ [see Eq. (396)] and by $\tilde{\Gamma}_{1}$ in the case of $d \tilde{\Gamma}_{2} / d h_{0}$ [see Eq. (397)]. The fact that $\tilde{\Gamma}_{1}$, which is proportional to $\left(J^{\perp}\right)^{2}$ in leading order according to Eq. (393), decreases for larger $c^{2}$, and $\tilde{\Gamma}_{2}$, which contains a term $\propto\left(J^{z}\right)^{2}$, see Eq. (394), increases with $c^{2}$, explains the observed behavior.

Finally, we discuss how the renormalized $g$ factor is affected by the anisotropy, see Fig. 17. For growing $c^{2}$, the

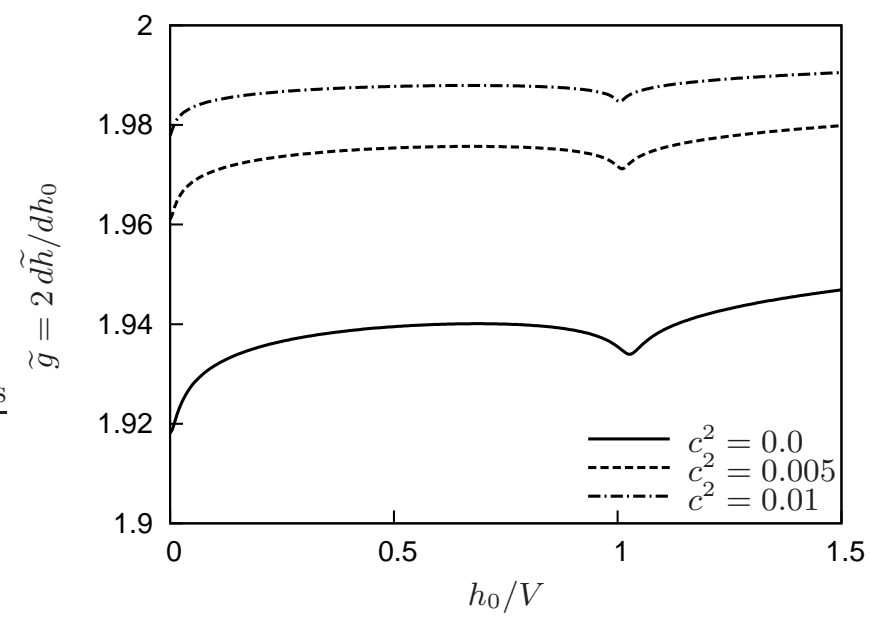

FIG. 17: The renormalized $g$ factor $\tilde{g}=2 d \tilde{h} / d h_{0}$ as function of the magnetic field at $V=10^{-4} D$ for the isotropic Kondo model (solid line) and the anisotropic Kondo model with two different values of $c^{2}=\left(J^{z}\right)^{2}-\left(J^{\perp}\right)^{2}$ (dashed and dash-dotted lines). The Kondo temperature $T_{K}=10^{-8} D$ is the same in all cases.

deviation of $\tilde{g}$ from the unrenormalized value 2 becomes smaller. The reason has already been discussed above: Like the renormalized field $\tilde{h}$, the $g$ factor (398) is in first order given by the renormalization of the coupling $J^{z}$ which is determined by $\left(J^{\perp}\right)^{2}$ and is therefore decreased for positive $c^{2}$. Also the prefactors of the logarithmic terms depend on $\left(J^{\perp}\right)^{2}$ and therefore become smaller for growing $c^{2}$.

\section{SUMMARY}

We have presented a systematic and analytic weakcoupling analysis in two-loop for a generic quantum dot coupled to reservoirs via spin or orbital fluctuations. We have used a nonequilibrium RG formalism in Liouville space (or, equivalently, on the Keldysh contour), which is a natural and formally exact generalization of conventional poor man scaling methods. The essential difference between the RTRG-FS method and other RG formalisms in nonequilibrium $\frac{19,20,24}{2}$ is the fact that RTRG-FS ana- 
lyzes the dynamics of the reduced dot density matrix in Laplace space by calculating an effective dot Liouvillian. In each step of the RG approach an effective kinetic equation is obtained. The RG equations are coupled differential equations for the kernel determining this kinetic equation and for the vertices in Liouville space. The structure in Liouville space is essential for the generation of dissipation and to obtain the transport relaxation and dephasing rates together with the transport oscillation frequencies of the various exponentially damped modes (rather than quantities of quantum decay theory, which describe the decay of a discrete dot state into the reservoir continuum). Technically, it is known from the Keldysh formalism ${ }^{37}$ that dissipation (or the collision term of a quantum Boltzmann equation) is generated by diagrams connecting the upper with the lower part of the Keldysh contour. Therefore, to get the correct transport rates and the precise position of resonances from the oscillation frequencies, it is essential to include these contributions in the theory and, at the same time, combine it with RG methods to get rid of logarithmic divergencies. We note that the RTRG-FS formalism is not based on the usual Keldysh formalism as, e.g., methods based on slave particles ${ }^{20}$ or nonequilibrium functional RG methods ${ }^{28}$ Since the unperturbed part of the Hamiltonian contains strong interactions, the RTRG-FS integrates out only the noninteracting reservoirs but takes the correlation effects on the quantum dot exactly into account. This leads to a natural formalism in terms of Liouville operators and vertices in Liouville space, which we have combined with quantum field theoretical methods of diagrammatic representations and RG. In this sense, we have combined the advantages of Liouville operator techniques (providing very compact notations for the time evolution of the reduced density matrix in Laplace space), diagrammatic methods (to identify and calculate irreducible blocks leading to the oscillation frequencies and the relaxation/dephasing rates), and RG methods (reorganizing the perturbation theory in such a way that logarithmic divergencies are absent). In particular, it is possible to prove generically in all orders of perturbation theory and within all truncation schemes that the cutoff scales for the vertices are the physical relaxation and dephasing rates of the exponentially decaying modes of the reduced density matrix of the dot. This provides a reliable calculation of the line shape at resonances. In addition, one obtains the kernel of the kinetic equation in Laplace space from which the time evolution into the stationary state can be calculated. It is important to note that the possibility to calculate the time evolution is closely related to the fact that we obtain a systematic theory for the line shape at resonances, since the oscillation frequencies and the relaxation/dephasing rates governing the exponential decay of the time evolution are the same scales which determine the resonance positions and the cutoff of logarithmic divergencies at resonances for stationary quantities such as, e.g., the magnetization or the conductance.
In comparism to previous formulations of RTRG (Refs. 25 27) (where the physics of cutoff scales by relaxation/dephasing modes was also included), the recently proposed RTRG-FS method ${ }^{17}$ is a formulation in pure Matsubara frequency space and avoids the need of the Keldysh indices after a first discrete RG step where the symmetric part of the Fermi distribution is integrated out. The main advantage of this development is the possibility to prove that the perturbation theory in the renormalized vertices on the r.h.s. of the RG equation is a well defined series in the weak-coupling regime. Essential for this proof is the fact that the imaginary part of the denominators of all resolvents consists of a sum of strictly positive Matsubara frequencies (bounded from above by the cutoff), the cutoff scale $\Lambda$, and some positive relaxation or dephasing rate $\tilde{\Gamma}_{i}$. As a consequence, it is possible to solve the RG equations analytically in the weakcoupling regime by a systematic perturbative expansion in the renormalized couplings. In this paper, we have described this procedure for a generic quantum dot in the Coulomb blockade regime where spin and/or orbital fluctuations dominate the transport properties. We have included all two-loop contributions to calculate physical quantities up to the first logarithmic contribution at resonances. We have shown that the latter are well defined after renormalization and can be treated within perturbation theory in the renormalized couplings.

We have applied the formalism to the anisotropic Kondo model at finite magnetic field and finite bias voltage. We calculated stationary properties (magnetic susceptibility and conductance) and the parameters characterizing the exponentially damped modes of the time evolution (renormalized magnetic field, spin relaxation and dephasing rate). In comparism to the case of zero magnetic field (which was treated within flow equation methods already in Ref. 24 for the isotropic Kondo model), the central issue is the calculation of logarithmically enhanced contributions at the resonance positions $\tilde{h} \approx V$ and $\tilde{h} \ll V$. The one-loop leading order theory of Ref. 20 was able to obtain these logarithmic contributions but it was not shown how to include microscopically the cutoff scales from relaxation and dephasing modes into the RG formalism. Furthermore, the renormalization of the magnetic field was not taken into account and the calculation was restricted to the stationary values of the magnetic susceptibility and the conductance (the spin relaxation and dephasing rates were calculated only perturbatively in the bare coupling and in the absence of a magnetic field in Ref. 29). In this paper, we have included the renormalization of the magnetic field in $O(J)$ and $O\left(J^{2} \ln \right)$ together with the spin relaxation and dephasing rate in $O\left(J^{2}\right)$ and $O\left(J^{3} \ln \right)$. This provides a consistent theory for the resonance position and the line shape at resonance, and answers the question which rate occurs in the various logarithmic terms. For stationary quantities, the obvious physical result that the spin dephasing rate cuts off the logarithmic contributions is obtained. For the rates and the renormalized field itself, 
it turns out that the spin relaxation rate determines the resonant line shape for the spin dephasing rate and the $g$ factor, whereas the spin dephasing rate controls the resonant line shape for the spin relaxation rate. In addition, it turns out that the logarithmic enhancements or suppressions at the resonance $\tilde{h}=V$ are quite different for the various physical quantities regarding the symmetry for $\tilde{h}>V$ and $\tilde{h}<V$. The $g$ factor shows a symmetric suppression, the conductance an asymmetric enhancement, the suspectibility shows only an enhancement for $\tilde{h}<V$, the derivative $\frac{d \tilde{\Gamma}_{1}}{d h_{0}}$ of the spin relaxation rate only an enhancement for $V>\tilde{h}$, and the corresponding quantity for the spin dephasing rate only a suppression for $V<\tilde{h}$. We proposed an experimental setup with a weakly coupled probe lead to measure the logarithmic suppression of the renormalized $g$ factor as function of voltage $V$ or magnetic field $h_{0}$. This has to be contrasted to the usual measurement of the $g$ factor at resonance $V=\tilde{h}$ from the position of the cotunnelingand Kondo-enhanced conductance peak, which provides the $g$ factor only for one special value of the voltage. In the anisotropic case, we find the obvious result that all logarithmic contributions involving the transverse coupling are suppressed for increasing longitudinal coupling since the renormalization of the transverse coupling is reduced. However, for the magnetic susceptibility this does not hold since the transverse coupling cancels out in the prefactor of the logarithmic terms. Therefore, the logarithmic enhancements become larger for the susceptibility if one increases the longitudinal coupling. This result might be helpful to find Kondo physics in transport through single molecular magnets, where the transverse coupling can be smaller than the longitudinal one. Concerning the line shape at resonance when increasing the longitudinal coupling, it turns out that the logarithmic features become less pronounced if the spin dephasing rate determines the line shape, whereas it becomes sharper when the spin relaxation rate is involved. The reason is that the spin dephasing rate involves a voltageinduced term $\sim V\left(J^{z}\right)^{2}$, which increases with increasing longitudinal coupling, whereas the spin relaxation rate involves only the transverse coupling in order $O\left(J^{2}\right)$.

\section{LIST OF SYMBOLS}

In this section we provide a complete list of all symbols used in this paper. We present two tables, one for the symbols used in Sec. II of the generic case, and one for Sec. III where the method is applied to the Kondo model.

The table for the symbols used in Sec. II is given by

\begin{tabular}{l|l|l} 
Symbol & What the symbol means & Ref. \\
\hline$H$ & Hamiltonian & $(10)$ \\
$H_{r e s}$ & reservoir Hamiltonian & $(11)$ \\
$H_{S}$ & dot Hamiltonian & $(11)$ \\
$V$ & coupling dot $\leftrightarrow$ reservoir & $\left(\frac{13}{10}\right)$ \\
$H_{0}$ & $H_{r e s}+H_{S}$ & frequency \\
$\omega$ & (13)
\end{tabular}

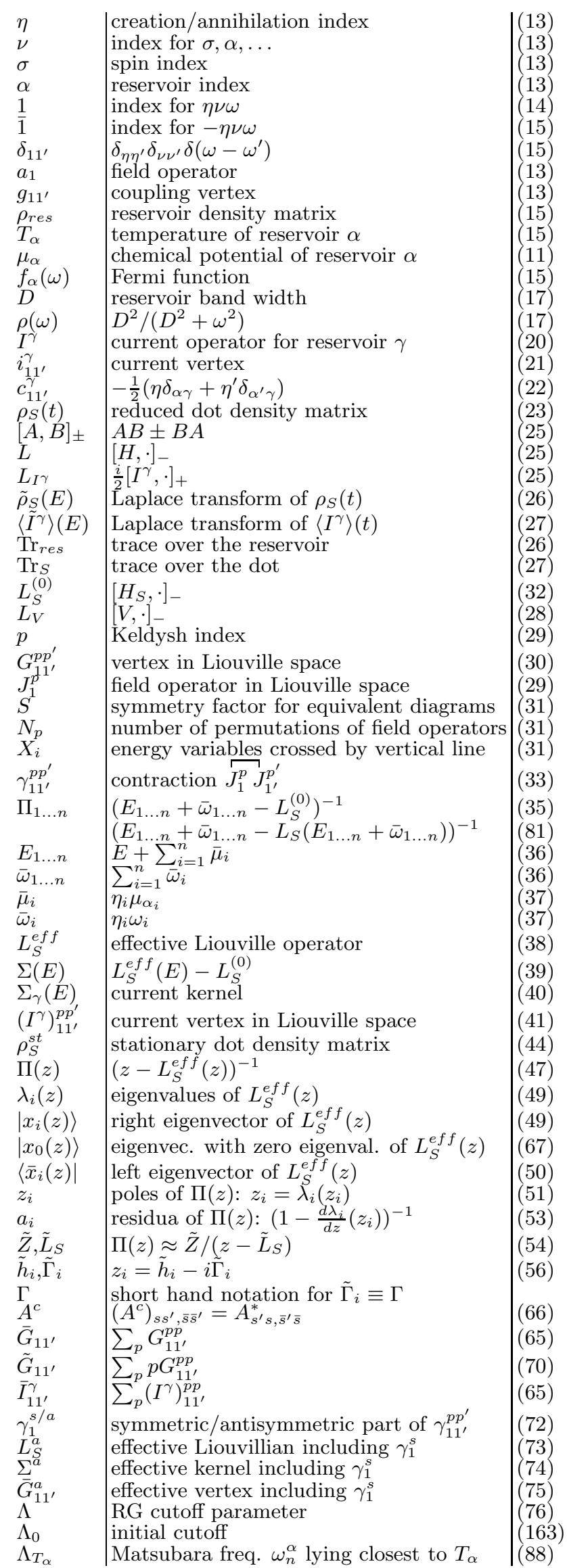




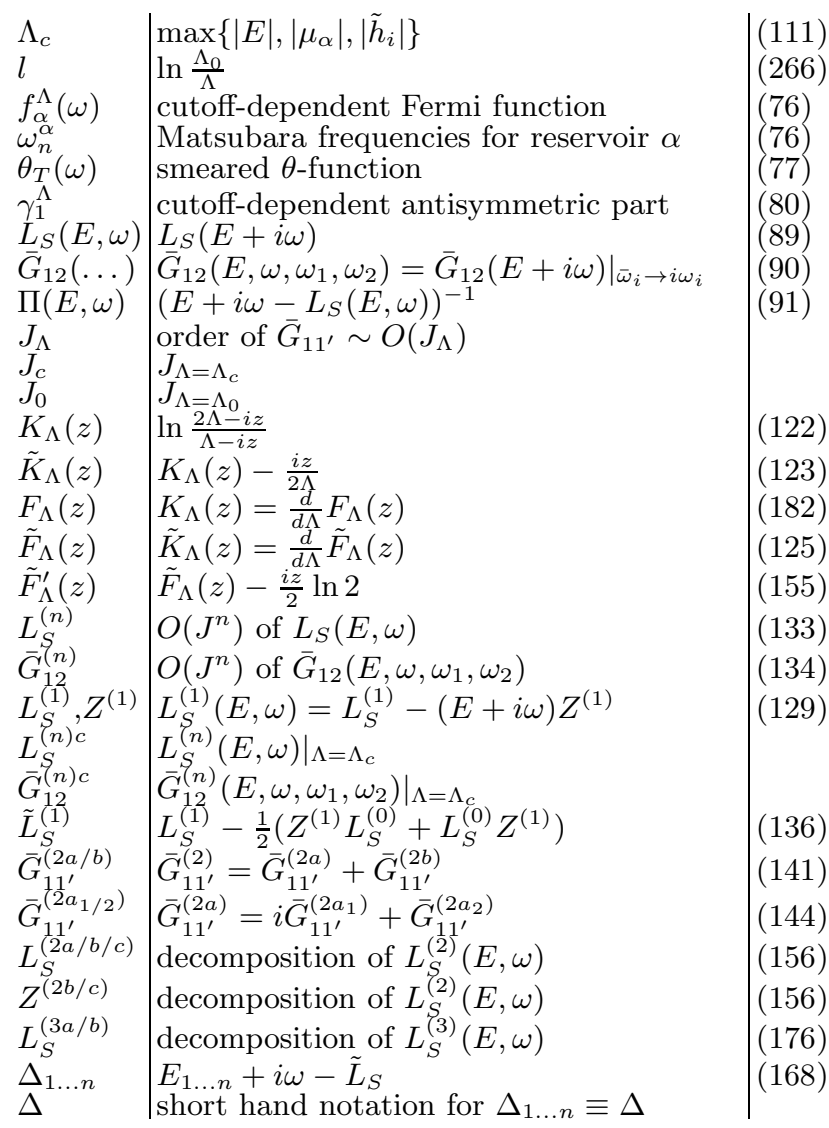

The table for the symbols used in Sec. III is given by

\begin{tabular}{|c|c|}
\hline \multirow{2}{*}{\multicolumn{2}{|c|}{$\frac{\text { Symbo }}{h_{0}}$}} \\
\hline & \\
\hline$\underset{\sim}{h}$ & $\left.-\frac{1}{2}\left(J_{\alpha}^{z}-\left(J_{\alpha}^{z}\right)_{0}\right)\right) h_{0}$ \\
\hline$\tilde{h}$ & renormalized magnetic field \\
\hline$\tilde{\Gamma}_{1}$ & spin relaxation rate \\
\hline$\tilde{\Gamma}_{2}$ & in dephasing rate \\
\hline & in operat \\
\hline$\left(J_{i \alpha^{\prime}}^{i}\right)_{0}$ & re exchange couplings $(i=x, y, z, \perp)$ \\
\hline $\begin{array}{l}J_{\alpha \alpha^{\prime}}^{\imath} \\
J^{z / \perp}\end{array}$ & $\begin{array}{l}\text { renormalized exchange couplings } \\
J^{z / \perp}=2 \sqrt{x_{0} x_{0}} J^{z / \perp}\end{array}$ \\
\hline$J_{\alpha}^{z / \perp}$ & $J_{\alpha \alpha}^{\alpha \alpha^{\prime}}+$ \\
\hline$J_{\text {nd }}^{z / \perp}$ & $J_{L R}^{z / \perp}=J_{R L}^{z / \perp}$ \\
\hline $\begin{array}{l}x_{\alpha} \\
I_{\gamma, z / \perp}\end{array}$ & factor for coupling to reservoir $\alpha$ \\
\hline $\begin{array}{l}J_{\alpha \alpha^{\prime}} \\
c_{\alpha \alpha^{\prime}}^{\gamma}\end{array}$ & \\
\hline$T_{K}^{\alpha \alpha^{\prime}}$ & Kondo temperature \\
\hline$T_{K}^{\prime}$ & $\sqrt{J_{0}^{\perp}} T_{K}$ \\
\hline$c^{2}$ & $\frac{1}{4} \operatorname{Tr}\left(\left(\hat{J}^{z}\right)^{2}-\left(\hat{J}^{\perp}\right)^{2}\right)$ \\
\hline $\begin{array}{l}V \\
L^{ \pm}\end{array}$ & $\begin{array}{l}\text { voltage } \\
L^{+} A=S A, L^{-} A=-A S\end{array}$ \\
\hline $\bar{L}^{a}$ & $\frac{3}{4} \cdot \mathbf{1}+\underline{\underline{L}}^{+} \cdot \underline{L}^{-}$ \\
\hline$L^{b}$ & $\frac{1}{4} \cdot 1-\underline{L}^{+} \cdot \underline{L}^{-}$ \\
\hline$L^{c}$ & $\frac{1}{2} \cdot \mathbf{1}+2 L^{+z} L^{-z}$ \\
\hline$L^{h}$ & $L^{+z}+L^{-z}$ \\
\hline$\underline{L}_{1}$ & $\frac{1}{2}\left(\underline{L}^{+}-\underline{L}^{-}-2 i \underline{L}^{+} \times \underline{L}^{-}\right)$ \\
\hline $\begin{array}{l}\underline{L}_{2} \\
\underline{L}_{3}\end{array}$ & $\begin{array}{l}-\frac{1}{2}\left(\underline{L}^{+}+\underline{L}^{-}\right) \\
\frac{1}{2}\left(\underline{L}^{+}-\underline{L}^{-}+2 i \underline{L}^{+} \times \underline{L}^{-}\right)\end{array}$ \\
\hline $\overrightarrow{L_{ \pm}^{i}}$ & $L^{i x \bar{x}} \pm i L^{\overline{i y}} ; i=\overline{1,2}, 3, \overline{ \pm}$ \\
\hline$L_{ \pm}^{4}$ & $L_{ \pm}^{2} \pm\left(L_{ \pm}^{+} L^{-z}+L^{+z} L_{ \pm}^{-}\right)$ \\
\hline & $\mid L_{ \pm}^{2} \mp\left(L_{ \pm}^{\mp} L^{-z}+L^{+z} L_{ \pm}^{-}\right)$ \\
\hline
\end{tabular}

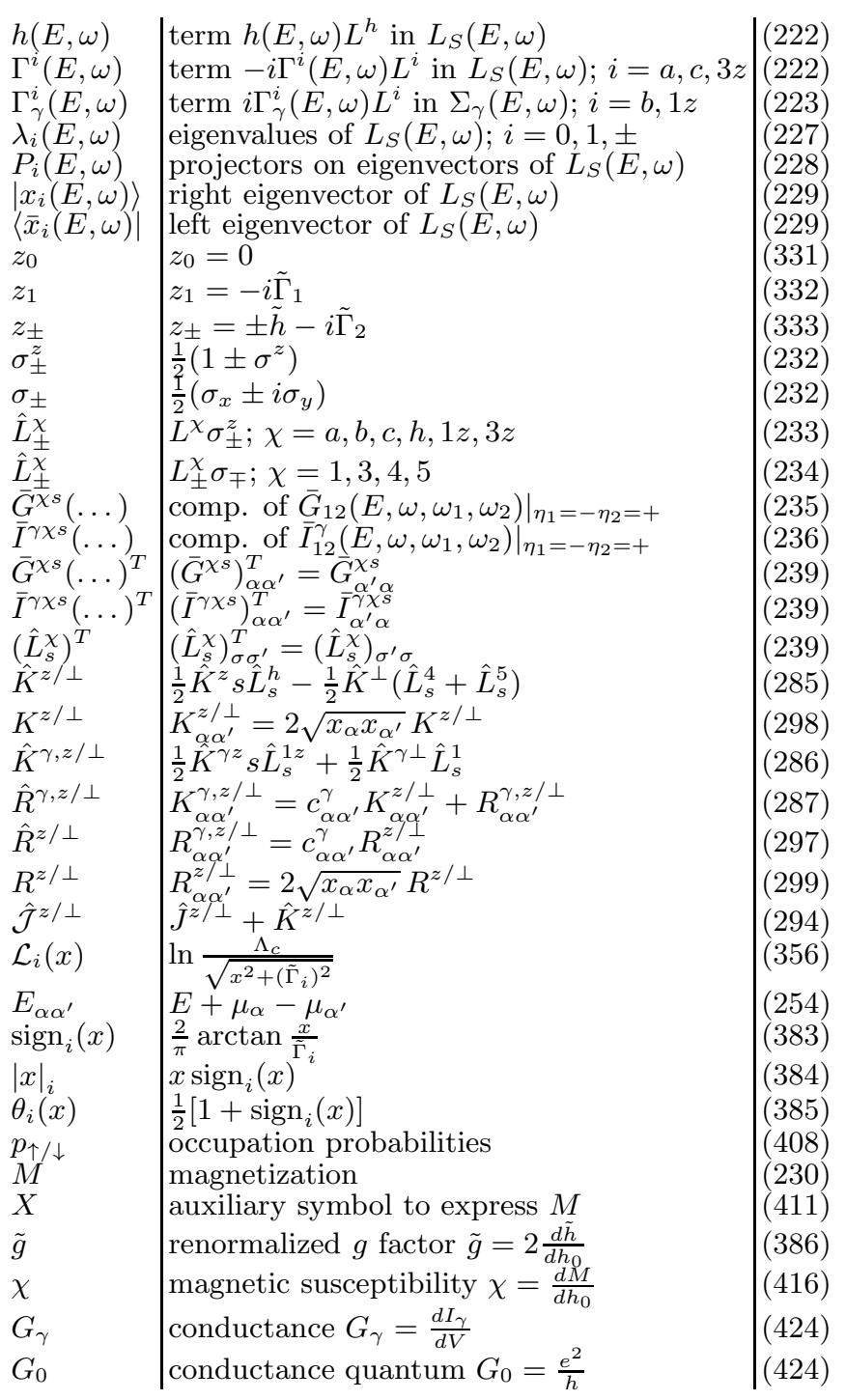

\section{Acknowledgments}

We thank N. Andrei, L. Glazman, S. Jakobs, S. Kehrein, H. Kroha, J. König, T. Korb, V. Koerting, M. Kurz, V. Meden, J. Paaske, M. Pletyukhov, A. Rosch, D. Schuricht, M. Wegewijs, P. Wölfle, and A. Zawadowski for valuable discussions. This work was supported by the DFG-Forschergruppe 723, the VW Foundation, and the Forschungszentrum Jülich via the virtual institute IFMIT.

\section{APPENDIX A: SECOND-ORDER RG}

In this appendix, we prove that the effect of the RG terms (149) and (150) is the replacement $F_{\Lambda}(z) \rightarrow F_{\Lambda}^{\prime}(z)$ in Eq. (152), with $F_{\Lambda}^{\prime}(z)$ defined by Eq. (155).

First we consider the term (150). The terms of $O\left(\frac{\Delta}{\Lambda} J^{3}\right)$ can be extracted by using the expansion (151) for the 
integral over the resolvent and considering only the terms of $O(1)$ and $O\left(\frac{\Delta}{\Lambda}\right)$ in this expansion,

$$
i \int_{0}^{\Lambda} d \omega_{2} \Pi\left(E_{12}, \Lambda+\omega+\omega_{2}\right) \approx \ln (2)+\frac{i}{2 \Lambda} z_{12},
$$

where we have defined

$$
z_{12} \equiv\left(E_{12}+i \omega-L_{S}^{(0)}\right)
$$

Inserting this in Eq. (150) for the two independent integrals over the resolvents and collecting the terms in $O\left(\frac{\Delta}{\Lambda} J^{3}\right)$ leads to the result

$$
\text { (150) } \rightarrow
$$

$$
-\frac{\ln (2)}{2 \Lambda}\left\{\bar{G}_{12}^{(1)} z_{12} \bar{G}_{\overline{2} 3}^{(1)} \bar{G}_{\overline{3} \overline{1}}^{(1)}+\bar{G}_{12}^{(1)} \bar{G}_{\overline{2} 3}^{(1)} z_{13} \bar{G}_{\overline{3} \overline{1}}^{(1)}\right\} .
$$

Next we consider the term (149) and use for $\bar{G}^{(2 b)}$ the result (142), which gives

$$
\begin{aligned}
\bar{G}_{\overline{2} \overline{1}}^{(2 b)}\left(E_{12}, \Lambda+\right. & \left.\omega+\omega_{2},-\omega_{2},-\Lambda\right)= \\
= & \bar{G}_{\overline{2} 3}^{(1)} \ln \frac{2 \Lambda-i z_{13}}{\Lambda} \bar{G}_{\overline{3} \overline{1}}^{(1)} \\
& \quad-\bar{G}_{\overline{1} 3}^{(1)} \ln \frac{\Lambda+\omega_{2}-i z_{23}}{\Lambda} \bar{G}_{\overline{3} \overline{2}}^{(1)},
\end{aligned}
$$

and

$$
\begin{aligned}
\bar{G}_{12}^{(2 b)}\left(E, \omega, \Lambda, \omega_{2}\right) & =\bar{G}_{13}^{(1)} \ln \frac{2 \Lambda-i z_{13}}{\Lambda} \bar{G}_{\overline{3} 2}^{(1)} \\
& -\bar{G}_{23}^{(1)} \ln \frac{\Lambda+\omega_{2}-i z_{23}}{\Lambda} \bar{G}_{\overline{3} 1}^{(1)}
\end{aligned}
$$

Equations (A4) and (A6) are independent of the integration variable $\omega_{2}$, and we can replace the logarithm by

$$
\ln \frac{2 \Lambda-i z_{13}}{\Lambda} \approx \ln (2)-\frac{i}{2 \Lambda} z_{13} .
$$

Inserting Eqs. (A4) and (A6) in (149), using Eq. (A1), and collecting all terms of $O\left(\frac{\Delta}{\Lambda} J^{3}\right)$, we find

$$
\begin{aligned}
& \text { (149) from (A4) and (A6) } \rightarrow \\
& \frac{\ln (2)}{2 \Lambda}\left\{\bar{G}_{12}^{(1)} z_{12} \bar{G}_{\overline{2} 3}^{(1)} \bar{G}_{\overline{3} \overline{1}}^{(1)}-\bar{G}_{12}^{(1)} \bar{G}_{\overline{2} 3}^{(1)} z_{13} \bar{G}_{\overline{3} \overline{1}}^{(1)}\right. \\
& \left.-\bar{G}_{13}^{(1)} z_{13} \bar{G}_{\overline{3} 2}^{(1)} \bar{G}_{\overline{2} \overline{1}}^{(1)}+\bar{G}_{13}^{(1)} \bar{G}_{\overline{3} 2}^{(1)} z_{12} \bar{G}_{\overline{2} \overline{1}}^{(1)}\right\}=0,(
\end{aligned}
$$

i.e., the terms cancel each other.

In contrast, Eqs. (A5) and A7 are not independent of the integration variable $\omega_{2}$. When inserted in Eq. (149), we need the integral $\left(z_{i j}\right.$ is replaced by its eigenvalue in this equation)

$$
\begin{aligned}
\int_{0}^{\Lambda} d \omega_{2} & \frac{1}{\Lambda+\omega_{2}-i z_{12}} \ln \frac{\Lambda+\omega_{2}-i z_{23}}{\Lambda} \approx \\
\approx & \frac{1}{2} \ln ^{2}(2)-i \frac{\ln (2)}{2 \Lambda} z_{12}+\frac{i}{2 \Lambda}\left(z_{12}-z_{23}\right)
\end{aligned}
$$

where we have expanded up to linear order in $\frac{z_{i j}}{\Lambda}$. This gives

(149) from (A5) and (A7) $\rightarrow$

$$
\begin{array}{r}
\rightarrow \frac{\ln (2)}{2 \Lambda}\left\{\bar{G}_{12}^{(1)} z_{12} \bar{G}_{\overline{1} 3}^{(1)} \bar{G}_{\overline{3} \overline{2}}^{(1)}+\bar{G}_{23}^{(1)} \bar{G}_{\overline{3} \overline{1}}^{(1)} z_{12} \bar{G}_{\overline{2} \overline{1}}^{(1)}\right\} \\
-\frac{1}{2 \Lambda}\left\{\bar{G}_{12}^{(1)} z_{12} \bar{G}_{\overline{1} \overline{3}}^{(1)} \bar{G}_{\overline{3} \overline{2}}^{(1)}+\bar{G}_{23}^{(1)} \bar{G}_{\overline{3} 1}^{(1)} z_{12} \bar{G}_{\overline{2} \overline{1}}^{(1)}\right. \\
\left.\quad-\bar{G}_{12}^{(1)} \bar{G}_{\overline{1} 3}^{(1)} z_{23} \bar{G}_{\overline{3} \overline{2}}^{(1)}-\bar{G}_{23}^{(1)} z_{23} \bar{G}_{\overline{3} 1}^{(1)} \bar{G}_{\overline{2} \overline{1}}^{(1)}\right\}
\end{array}
$$

Using the antisymmetry property (97), we see that the second term is zero and the first term agrees with Eq. (A3), leading to the final result

$$
\begin{aligned}
& (149)+(150) \rightarrow \\
& -\frac{\ln (2)}{\Lambda}\left\{\bar{G}_{12}^{(1)}\left(E_{12}+i \omega-L_{S}^{(0)}\right) \bar{G}_{\overline{2} 3}^{(1)} \bar{G}_{\overline{3} \overline{1}}^{(1)}+\right. \\
& \left.\quad+\bar{G}_{12}^{(1)} \bar{G}_{\overline{2} 3}^{(1)}\left(E_{13}+i \omega-L_{S}^{(0)}\right) \bar{G}_{\overline{3} \overline{1}}^{(1)}\right\}
\end{aligned}
$$

Finally, using the leading-order RG equations (115) or (116), we see that Eq. (A11) can be written in the form

$$
\begin{aligned}
& \sqrt{149)}+(150) \rightarrow \\
& -\frac{\ln (2)}{2} \frac{d}{d \Lambda}\left\{\bar{G}_{12}^{(1)}\left(E_{12}+i \omega-L_{S}^{(0)}\right) \bar{G}_{\overline{2} \overline{1}}^{(1)}\right\}
\end{aligned}
$$

i.e., a similiar term to Eq. (152) results, which proves the replacement (155).
1 A. C. Hewson, The Kondo Problem to Heavy Fermions, Cambridge University Press (1997).

${ }^{2}$ L. I. Glazman and M. E. Raikh, Sov. Phys. JETP Lett. 47, 452 (1988); T. K. Ng and P. A. Lee, Phys. Rev. Lett. 61, 1768 (1988).

3 D. Goldhaber-Gordon et al., Nature 391, 156 (1998); S. M. Cronenwett et al., Science 281, 540 (1998); F. Simmel et al., Phys. Rev. Lett. 83, 804 (1999).
4 N. Andrei, Phys. Rev. Lett. 45, 379 (1980); P. B. Wiegmann, JETP Lett. 31, 364 (1980); A.M. Tsvelick and P. B. Wiegmann, Adv. in Physics 32, 453 (1983);

5 A. W. W. Ludwig and I. Affleck, Phys. Rev. Lett. 67, 3160 (1991); I. Affleck and A. W. W. Ludwig, Phys. Rev. B 48, 7297 (1993).

${ }^{6}$ K. G. Wilson, Rev. Mod. Phys. 47, 773 (1975).

7 T. A. Costi, A. C. Hewson, and V. Zlatic, J. Phys.: Con- 
dens. Matter 6, 2519 (1994).

8 P. W. Anderson, J. Phys. C 3, 2436 (1970); F. D. M. Haldane, Phys. Rev. Lett. 40, 416 (1978).

9 F. B. Anders and A. Schiller, Phys. Rev. Lett. 95, (2005) 196801.

${ }^{10}$ F. B. Anders, arXiv:0802.0371,

11 S. Weiss, J. Eckel, M. Thorwart, and R. Egger, Phys. Rev. B 77, (2008) 195316.

12 N. Andrei and G. Palacios, private communication.

13 P. Coleman, C. Hooley, and O. Parcollet, Phys. Rev. Lett. 86, (2001) 4088.

14 A. Kaminski, Yu. V. Nazarov, and L. I. Glazman, Phys. Rev. B 62, (2000) 8154.

15 A. Rosch, J. Kroha, and P. Wölfle, Phys. Rev. Lett. 87, (2001) 156802.

16 D. V. Averin and A. A. Odintsov, Phys. Lett. A 140, 251 (1989); D. V. Averin and Yu. V. Nazarov, Phys. Rev. Lett. 65, 2446 (1990).

17 H. Schoeller, Eur. Phys. J. Special Topics 168, 179 (2009).

18 E. Sela, H. S. Sim, Y. Oreg, M. E. Raikh, and F. von Oppen, Phys. Rev. Lett. 100, 056809 (2008).

19 L. I. Glazman and M. Pustilnik, in Nanophysics: Coherence and Transport (H. Bouchiat et al., Elsevier, 2005) p. 427; note that a typing error occured in Eq. (5.63) of this reference, the coefficient in front of the $\theta$-function must be 3 and not 2 .

20 A. Rosch, J. Paaske, J. Kroha, and P. Wölfle, Phys. Rev. Lett. 90, 076804 (2003); ibid., J. Phys. Soc. Jpn. 74, 118 (2005).

21 C. Romeike, M. R. Wegewijs, W. Hofstetter, and H. Schoeller, Phys. Rev. Lett. 96, 196601 (2006).

${ }^{22}$ H. B. Heersche et al., Phys. Rev. Lett. 96, 206801 (2006); M.H. Jo et al., Nano Lett. 6, 2014 (2006).

23 F. Wegner, Ann. Phys. (Leipzig) 3, 77 (1994); S. D. Glazek and K. G. Wilson, Phys. Rev. D 48, 5863 (1993); ibid., 49, 4214 (1994).

24 S. Kehrein, Phys. Rev. Lett. 95, 056602 (2005); P. Fritsch and S. Kehrein, arXiv:0811.0759

25 H. Schoeller and J. König, Phys. Rev. Lett. 84, 3686 (2000).

26 H. Schoeller, in Low-Dimensional Systems, ed. T. Brandes, Lect. Notes Phys. (Springer, 2000), p. 137.

27 T. Korb, F. Reininghaus, H. Schoeller, and J. König, Phys. Rev. B 76, 165316 (2007).

28 S. G. Jakobs, V. Meden, and H. Schoeller, Phys. Rev. Lett. 99, 150603 (2007).

29 J. Paaske, A. Rosch, J. Kroha, and P. Wölfle, Phys. Rev. B 70, 155301 (2004).

30 J. Koch, M. E. Raikh, and F. von Oppen, Phys. Rev. Lett. 96, 056803 (2006).

31 N. Andrei, K. Furuya, and J. H. Lowenstein, Rev. of Modern Physics 55, 331 (1983).

32 J. Paaske, A. Rosch, and P. Wölfle, Phys. Rev. B 69, 155330 (2004).

33 M. Garst, P. Wölfle, L. Borda, J. von Delft, and L. I. Glazman, Phys. Rev. B 72, 205125 (2005).

34 J. Zinn-Justin, Quantum Field Theory and Critical Phenomena, (Cambridge University Press, 2002).

35 B. Doyon and N. Andrei, Phys. Rev. B 73, 245326 (2006).

36 J. Solyom and A. Zawadowski, J. Phys. F: Metal Phys. 4, 80 (1974).

37 L. V. Keldysh, Yh. Eksp. Teor. Fiz. 47, 1515 (1964) [Sov. Phys. JETP 20, 1018 (1965)]; L. P. Kadanoff and G. Baym, "Quantum Statistical Mechanics" (Benjamin, New York, 1962).

38 R. Leturcq et al., Phys. Rev. Lett. 95, 126603 (2005).

39 Our convention is that all terms on the r.h.s. of the $\mathrm{RG}$ equation which are of $O\left(G^{2}\right)\left[O\left(G^{3}\right)\right]$ are called one-loop (two-loop) terms. For the RG of the vertices, this is in agreement with the conventional classification but the two RG diagrams in Fig. 4 for the Liouvillian are also called two-loop and three-loop terms in the literature. 